\title{
IL LUNGO CAMMINO DEI MERCANTI DI SAPIENZA. LE ORIGINI DELL'UNIVERSITÀ DI PAVIA NELLA STORIOGRAFIA DAL XIV AL XX SECOLO
}

\author{
Nota del m.e. DARIO MANTOVANI (*)
}

(Adunanza del 23 giugno 2011)

SuNTO. - Il saggio offre un contributo alla storia della storiografia sull'Università di Pavia. Vengono presi in esame non solo i testi che a partire dal Settecento si sono esplicitamente rivolti a studiare la storia dell'Università, ma tutte le testimonianze di una coscienza storica intorno alle origini e alla storia dell'Università, fin dalla sua fondazione viscontea nel 1361. Particolare attenzione è rivolta ai tre racconti relativi all'origo: si dimostra come per lungo tempo in Europa si sia creduto alla fondazione da parte di Carlo Magno, contemporaneamente a Parigi, e si identifica il diffusore di questo racconto in Barthélemy de Chasseneuz, nel 1525. L'attribuzione della fondazione al Capitolare di Lotario dell'825 è un'idea recente, che è stata nutrita, con intenti diversi, dalla 'scoperta' - da parte degli storici del diritto tedeschi nel XIX secolo - della scuola di diritto longobardo a Pavia (attestata almeno dal X secolo) e dalle Celebrazioni del 1925.

ABSTRACT. - This paper offers a contribution to the history of historiography on the University of Pavia. The Author takes into account both the treaties explicitly dealing (since the $18^{\text {th }}$ Century) with the history of the University, but also all the evidence of a historical consciousness about the origins and history of the University; such a histori-

(*) Dipartimento di Giurisprudenza, Università di Pavia.

E-mail: dario.mantovani@unipv.it. Il testo appare anche in Almum Studium Papiense. Storia dell'Università di Pavia, 1, I, a c. di D. Mantovani, Cisalpino, Milano, 2012, pp. 29-82. 
cal consciousness started to appear in 1361, when the Visconti Family officially founded the University. Particular attention is paid to the three interpretations about the foundation (origo), which has been attributed to the Lombard Kings, to Charlemagne and to Lothair I. For a long time there was a widespread belief in Europe that the University of Pavia had been founded by Charlemagne, simultaneously with the University of Paris; the creator of this tradition (based on the history of Charlemagne written by Notker the Stammerer) was Barthélemy de Chasseneuz, in 1525. The attribution of the founding to Lothair in 825 is only a recent idea, which has been nourished, with different intentions, by the $19^{\text {th }}$ Century German legal historians who discovered a school of Lombard Law in Pavia (attested since at least the $10^{\text {th }}$ Century) and by the Celebrations held in Pavia in 1925.

\section{TRE ORIGINI PER UNA UNIVERSITÀ}

Quando nacque, nel 1361, lo Studium Papiense aveva già un passato infinito alle spalle, reminiscenza di un'esistenza anteriore forse mai vissuta, ma capace di influire sul presente. Autentica o fittizia, la sua origine antichissima sarà sempre (fino a oggi) uno specchio che ne ferma l'immagine e ne stabilisce una volta per tutte il rango, quasi con il medesimo valore giuridico di un privilegium imperiale.

Ogni origo si costruisce attraverso un racconto, tanto più complesso quanto più è lunga la storia di cui è l'ombra. Di origini, l'Università di Pavia ne vanta addirittura tre, attribuite ai re longobardi, a Carlo Magno, a Lotario I. Rintracceremo la genesi e le metamorfosi di questi racconti di fondazione, in un tragitto che ci porterà ad attraversare la coscienza storica di quanti, prima di noi, hanno ragionato sul passato - fabula o bistoria - dell'Ateneo. Quest'esplorazione sarà un primo contributo a una storia della storiografia universitaria pavese, che finora manca. ${ }^{1}$

L'obiettivo è di chiarire come si siano formati modelli interpretativi che tuttora fanno da sfondo, a volte inconsapevolmente, alla ricer-

1 Offrono utili notizie e valutazioni: Capsoni 1785, II, pp. V-XXIII, con una panoramica dal Seicento; poi i Cenni inediti di Bussedi 1864, p. 1 (PAVIA - BiblioteCA UnIVERsitaria, BUPv, Ticinesi, 212, p. 1); Corradi, in Memorie e Documenti, I, pp. IIIV; Zoncada, ivi, pp. 515-518; Franchi, in Statuti e Ordinamenti 1925, pp. V-XVI; prezioso per metodo e risultati Gabba 2000, pp. 37-42; specificamente sulle ricerche promosse dal Centro per la storia dell'Università di Pavia, vd. Guderzo 2011, pp. 248-265. Nella diversa prospettiva di un bilancio dei risultati e dell'indicazione di percorsi di ricerca, vd. Malamani 1980, pp. 291-299; Ferraresi 1986, pp. 58-75. 
ca; al contempo, le riflessioni sul passato elaborate a partire dal XIV secolo costituiscono esse stesse capitoli interessanti della vita dello Studium pavese. $^{2}$

Il tragitto si snoderà necessariamente lungo il filo di una pluralità di forme letterarie. Dagli atti ufficiali alle orazioni panegiriche, dalle allegazioni giuridiche ai cataloghi eruditi, dalle cronache alle storie e alle biografie, l'emergere di una coscienza del passato è imprevedibile, se non ci si accontenta di fermarsi ai trattati stesi esplicitamente in chiave storiografica, che sono del resto il portato di una stagione piuttosto tarda, che si può convenzionalmente fare iniziare con il Settecento. ${ }^{3}$

Alla pluralità di espressioni letterarie corrisponde naturalmente pluralità di intenti e di esiti. Al tempo stesso, le sfaccettature dei generi letterari lasciano intravvedere il farsi di una conoscenza storica più fondata, uno spostarsi di interessi, il sorgere di nuove domande, mentre altre perdono d'attrattiva. Le risposte tendono a rinnovarsi, ma anche a tornare ingenuamente alla ribalta o semplicemente a cadere in oblio: l'esempio più clamoroso, su cui avremo modo di insistere, è la fondazione da parte di Carlo Magno, che per secoli fu la versione più diffusa dell'origo Studii, per poi essere abbandonata senza rumore, sostituita dal Capitolare di Lotario dell' 825 , un documento intorno al quale - per una serie di circostanze avveratesi fra il XIX e il XX secolo - vennero a stringersi in una trama apparentemente nuova fili tante volte già tessuti.

Naturalmente, s'è dovuto compiere una selezione, non solo per ragioni di spazio. Il principale criterio selettivo è implicito nel tema. Questa esplorazione della coscienza storica assume come centro l'origo, lasciando in margine l'altra direttrice, cioè il modo in cui s'è venuta pensando e ricostruendo la storia dello Studium vero e proprio dal 1361 (non dei suoi preludi): la direttrice che Pietro Francesco Scarabelli - un medico del Seicento che avrebbe desiderato raccontarla - chiamò progressio. ${ }^{4} \mathrm{La}$ distinzione rende più leggibili continuità e fratture, e mette

2 Da quest'angolatura non saranno affrontati, se non per quanto indispensabile, i problemi di ricostruzione, ossia se la tale fonte possa o meno giustificare questa o quella ipotesi di origine.

3 Vd. il saggio di Frova 2012, qui presupposto.

4 Sulla sua Historia Gymnasii Ticinensis, che rimase solo un progetto («adumbrata historia»), vd. infra. 
in risalto la differenza stessa fra origo e progressio, di cui non sempre si tiene adeguatamente conto quando si ragiona in chiave storiografica. Tuttavia, siccome i due percorsi spesso si intrecciano, a volte persino nella pagina di uno stesso autore, s'è dato conto - per cenni, quasi in contrappunto - anche della seconda prospettiva, segnalando le tappe principali degli studi dedicati alla progressio successiva al 1361.

Se il vaglio è stato selettivo, il numero delle testimonianze raccolte mostra che, a differenza di quel che di solito si ritiene, la riflessione sul passato è stata intensa e ha preceduto di molto i testi ben noti almeno a chi si occupa di storia dell'Università - con cui si fa in genere iniziare nel Settecento la storiografia sull'Ateneo pavese, cioè le vindiciae di Antonio Gatti e l'Elenchus di Giacomo Parodi (che sono i campioni, rispettivamente, delle indagini sull'origo e di quelle sulla progressio). Questo è il risultato più evidente del sondaggio.

Solo di un sondaggio, in effetti, si tratta, che presenta testi e autori quali punti di una linea che l'ampliamento delle indagini potrà confermare o modificare.

\section{IL TEMPO DEL MITO E IL TEMPO DELLA STORIA: PiETRO AZARIO ARCHETIPO DI UN'INTERPRETAZIONE}

Un bozzetto storico nascosto in un fitto repertorio di pareri giuridici resi dal professore primario di Diritto canonico Giovanni Battista Costa $(† 1615)$ condensa l'idea che all'inizio del Seicento si aveva del presente e del passato dell'Università di Pavia, allora chiamata Gymnasium Ticinense:

La gloria del Ginnasio Ticinense consta soprattutto di cinque aspetti, ossia la mitezza del clima; la salubrità dell'aria; l'abbondanza delle provviste; la prestanza dei professori in tutte le epoche; l'antichità, dal momento che gli annali fededegni testimoniano che fu istituito dall'augustissimo Carlo Magno re dei Franchi nell'anno del Signore 740 - come attestano [nei loro libri] il Cardinale Zabarella e Antonino Fiorentino - secondo le ragioni addotte dal Chasseneuz, [là] dove assevera che anche l'Università di Parigi ha avuto nello stesso tempo la sua nascita, così che la Scuola parigina e l'Accademia pavese devono dirsi coeve e contemporanee, entrambe famose, celeberrime, e nutrici di ingegni di successo.

Questo nostro Ginnasio fiorisce di poi grazie a numerosi privilegi, principalmente sotto Carlo IV Imperatore dei Romani nell'anno 1361, ed esiste un 
diploma pontificio, precisamente di Bonifacio IX dell'anno 1389, con cui in tutto e per tutto vengono concesse al Ginnasio di Pavia le immunità e le prerogative dei Ginnasi di Roma, Bologna, Parigi e di qualsiasi altra Università in favore dei professori e degli studenti.

Questa sorta di manifesto dell'Università di Pavia, affisso in apertura del parere giuridico del Costa, vale come premessa per tutelare l'immunità fiscale dei docenti, contro recenti tentativi d'eroderla da parte degli esattori spagnoli: la sua tesi è che le esenzioni facciano parte dello stipendio e garantiscano al docente il rimborso delle crescenti spese sostenute per la propria formazione e per acquistare libri, strumenti della professione. ${ }^{6}$

L'elenco dei pregi è disposto in una climax ascendente, il culmine è l'antichità, con il sigillo autorevole di Carlo Magno, prefigurazione di altri imperatori e papi benevoli. La storia (o il mito storico: basti considerare l'impossibile datazione al 740) fonda e si fonde con una pretesa giuridica, secondo una delle angolature più caratteristiche dell'uso del passato in età medievale e moderna. ${ }^{7}$

Tuttavia, nel bozzetto tratteggiato dal Costa si percepisce anche

5 Costa 1610, q. 236, pp. 299-300: «Gymnasij Ticinensis gloria quinque potissimum constat, nimirum, coeli clementia, salubritate aeris, fructuum copia, interpretum praestantia per omnes aetates, antiquitate, postquam fuisse institutum ab Augustissimo Carolo Magno Rege Francorum anno Domini 740 fidissimi testantur annales, teste Cardin. Zabarella in Clem. 1 de magistris quaestio 5, Anton. Florentino in sua historia parte 2 titulo 14 cap. $4 \$ 12$, cum adductis per Cassaneum in Catalogo de gloria mundi par. 10, consideratione 32, ubi asseverat quoque universitatem Parisiensem eodem tempore habuisse sua incunabula, ita ut schola Parisiensis et academia Papiensis dicantur coevae et contemporaneae, utraque famosa, percelebris, atque altrix felicissimorum ingeniorum. Floret deinceps hoc nostrum gymnasium conpluribus privilegijs, praecipue vero sub Carolo Quarto, Romanorum Imperatore anno 1361, extatque diploma Pontificium, scilicet Bonifacij IX, anno 1389, quibus in omnibus et per omnia conceduntur gymnasio Papiensi immunitates atque praerogativae gymnasi Romani, Bononiensis, Parisiensis et quarumcumque aliarum universitatum favore lectorum et studiosorum». Per gli autori citati dal Costa, vd. infra.

6 Ivi, q. 236, pp. 300-301: ciascuno può constatare come crescano di giorno in giorno le spese dei docenti «tum quoad studia scholastica peragenda, tum quoad lauream consequendam, tum etiam quoad decentem ac sufficientem librorum suppellectilem comparandam». Un analogo uso giuridico, pochi anni dopo, è compiuto da Ottaviano Picenardi, podestà di Pavia, in una lettera indirizzata al Senato di Milano, 8 maggio 1627 (Milano - ARChivio Di Stato, ASMi, Studi p.a., 158).

7 L'errore di datazione discende dal Chasseneuz (vd. infra). 
un'attenzione al tempo reale, rappresentato dalle condizioni di clima e di soggiorno e dall'eccellenza dei docenti «per omnes aetates». ${ }^{8}$

Le due dimensioni, della fabula e dell'bistoria, i due sguardi, quello anamnestico rivolto all'origo nobilitante e quello autoptico attento alle vicende concrete (ma che nell'origo trovano una garanzia), non maturano solo all'alba del Seicento.' S'intrecciano fin dalla fondazione dello Studium, nella cronaca del notaio Pietro Azario (1312-post 1367), pronto a stilarne l'atto di nascita, primo esponente di una serie di giuristi che a lungo coltiveranno la memoria dell'Università. La pagina che l'Azario dedica allo Studium è prossima alla conclusione del Liber gestorum in Lombardia che s'arresta al 1364, cronaca scritta «con ingegno vivido e capace di una corretta valutazione dei fatti» secondo il giudizio non invecchiato del Muratori. ${ }^{10}$

Il 27 ottobre 1361 (ma l'anno manca nell'Azario e potrebbe essere forse il 1362) $)^{11}$ una lettera di Galeazzo II porta una notizia alle città lombarde: a Pavia è aperta un'Università, uno Studium generale:

8 Il riferimento al clima favorevole, tuttavia, non è necessariamente realistico, essendo uno dei caratteri tipici degli elogi della città ideale fin dall'antichità greca $\mathrm{e}$ romana; vd. la rassegna delle laudes urbium da Platone al Medioevo in Classen 1980. Ritiene che i ripetuti elogi (da Opicino a Petrarca) indichino effettivamente che «la situazione climatica di Pavia dovette essere nel passato diversa dall'attuale» Cerri 1992, pp. $485-486$.

9 In generale, nell'ampia riflessione sull'uso del mito e della genealogia nella storiografia medievale e umanistica, vd. per tutti Bietenholz 1994, pp. 146-188; Spiegel 1997; Irace 2003, spec. pp. 13-75; Melville - Rehberg 2004; Bizzocchi 2009. Per il rapporto fra diritto e storiografia, rinvio ai classici Garin 1969, pp. 237-260; Dionisotti 1971, pp. 189-204 e Momigliano 1984, pp. 3-88.

10 Azario 1730, col. 293. Sull'Azario e la sua storiografia, vd. per tutti Cognasso, in Azario 1927, pp. I-XXXVIII; Nada Patrone 1962, pp. 740-742; Dale 2007, spec. pp. 178-195. Non è noto dove abbia compiuto gli studi giuridici.

11 La questione (già segnalata, ma non risolta dal Robolini 1836, V, 2, pp. 28-29; vd. anche Tiraboschi 1775, V, p. 59), merita un approfondimento. La data del 1361 viene indotta da quella - certa - del diploma di Carlo IV. Tuttavia, l'unico autore che riporti la lettera (ossia Azario 1730, cap. XII, col. $406=1927$, pp. 156-157) ne indica solo giorno e mese («XXVII Octobris») e poco prima annota di stare scrivendo nel 1362 («anno currente»), registrando la morte di papa Innocenzo VI avvenuta nel mese di ottobre di quell'anno (in realtà, 12 settembre): il che può suggerire che anche la lettera di Galeazzo II, riferita contestualmente e pure essa di ottobre, sia dello stesso 1362. La struttura non annalistica di questa parte del Liber gestorum, che offre un quadro delle imprese di Galeazzo Visconti, non esclude tuttavia che Azario - citando la lettera - stesse 
Galeazzo Visconti (signore) di Milano ecc., Vicario generale dell'Imperatore. Poiché abbiamo uno Studio nella città nostra di Pavia, tanto in Diritto canonico quanto in Diritto civile, nonché in Medicina e Fisica e Logica, e poiché abbiamo colà docenti idonei, vi incarichiamo di fare proclamare nelle vostre rispettive città, nei luoghi abituali, che ogni studente debba recarsi prontamente nella città nostra di Pavia, sotto pena da irrogare a nostro arbitrio. E se qualcuno si fosse recato in Studi altrui, lo si mandi a ricercare subito e sia obbligato a venire a Pavia. Infatti proprio nella città di Pavia abbiamo ottenuto i privilegi solenni di uno Studio Generale, con il potere e l'autorizzazione di tenere l'esame di dottorato in Decretali, in Leggi e in qualunque Facoltà. Milano, 27 ottobre. $^{12}$

L'attenzione che Azario rivolge al fatto - di cui è testimone oculare - s'iscrive nella sua visione del mondo, che era condizionata dall'essere osservatore delle campagne militari che devastavano il Nord dell'Italia, e alle quali il cronista non vedeva altra soluzione che il dominio dei Visconti. Quel che pensava di Pavia è racchiuso nella spiegazione che accoglieva del nome, «Papia parum pia dicta est»:.13 questa condizione etimologica di scarsa pietà caratterizzava la storia presente della città, facendo di Pavia un modello di decadenza che i Visconti dovevano risollevare attraverso la conquista, ch'era avvenuta due anni prima della fondazione dello Studium. ${ }^{14}$

fornendo una notizia retrospettiva (come fa Corio 1978, I, p. 805, che da lui dipende e dunque non vale come fonte autonoma per la datazione). Bisogna d'altra parte distinguere il problema della data della lettera di Galeazzo II dal problema dell'effettivo inizio dello Studio (aspetto, quest'ultimo, su cui si vedano le opinioni riferite da Belloni 1982, pp. 17-19). Anche se la lettera riportata dell'Azario fosse del 1362, nulla escluderebbe (ma niente confermerebbe) che l'inaugurazione fosse avvenuta l'anno precedente, ma che il cronista non disponesse dell'esemplare del primo avviso.

12 Azario 1730, cap. XIV, col. 406: «Galeaz Vicecomes Mediolani etc., Imperialis Vicarius generalis. Cum habeamus Studium in civitate nostra Papiae tam in iure canonico quam civili, et in Medicina, in physica et logica, habeamusque ibi doctores sufficientes, mandamus vobis quatenus proclamari faciatis in civitatibus vestris in locis consuetis, quod quilibet scholaris debeat ad Civitatem nostram Papiae statim accedere, sub pena nostro arbitrio auferenda. Et si quis ivisset ad aliena Studia, statim mittatur pro eis, et compellantur venire Papiam. In ipsa enim civitate Papiae aquisivimus privilegia solemnia Studii generalis, cum potestate et auctoritate dandi conventum in decretalibus, legibus, et qualibet facultate. Datum Mediolani, xxvii Octobris».

13 Azario 1730, cap. IX, col. $370=1927$, p. 112. L'attuale decadenza, che Azario attribuisce a disunione ed empietà, ben si accorda con la preminenza che egli assegna al ruolo antico di Pavia: Majocchi 2008, pp. 192-194.

14 Vd. Crotti 2012, pp. 219-228 e 240-246. Per la figura del frate Jacopo Bussolari, come esempio di religiosità "civica" studiata dal punto di vista della psicologia degli assediati, vd. Varanini 2007, pp. 12-13. 
Reinserita nello sfondo della narrazione dell'Azario, la lettera di Galeazzo II prende corpo: si rivela non documento che il cronachista s'accontenta di registrare, ma nucleo su cui converge la narrazione (e modello di tante future interpretazioni).

Innanzitutto, la scelta stessa di dare la parola a Galeazzo II attraverso la sua lettera lo rende protagonista e serve a sottolineare l'evergetismo del signore, che recluta docenti all'altezza («doctores sufficientes»): uno sforzo anche finanziario di cui s'era dubitato, ma che è stato confermato da ricerche recenti. ${ }^{15}$ La Cronaca dell'Azario offre poi un altro elemento di interpretazione: raramente Galeazzo II emetteva provvedimenti ad personam, mentre voleva che i podestà giudicassero attenendosi agli statuti e, nei casi non previsti, secondo il diritto romano. ${ }^{16} \mathrm{La}$ costruzione di uno stato regionale unitario, quale quello perseguito dai Visconti, passava per il rafforzamento della legalità. Il cronachista pare dunque suggerire che fu (anche) per formare quadri dirigenti dotati di cultura giuridica che i Visconti decisero un notevole investimento a carico delle casse pubbliche, secondo un modello - possiamo aggiungere - che differenziava perciò (anche) lo Studium pavese dall'Universitas delle origini bolognesi, che era invece aggregazione corporativa e privata di studenti che ingaggiavano il maestro.

Oltre a dispensare lusinghe, la lettera di Galeazzo II diramava un ordine perentorio: nessuno studente che abiti nello Stato visconteo può recarsi in altra Università, ad esempio a Bologna o a Padova, e se vi si fosse iscritto negli anni passati, deve immediatamente trasferirsi a Pavia,

15 Vd. gli importanti studi di Belloni 1982; Ead. 1985 e di Renzo Villata Massetto 2012, pp. 431-448. Cinquant'anni più tardi, Uberto Decembrio $(\dagger 1427)$ ne discorreva in modo analogo in un brano contenuto nel ms. Milano, Bibl. Ambr., B. 123 sup., trascritto da Hortis 1874 , p. 148, nt. 2. Il brano riporta lo Studium al comune denominatore della fabbrica e dell'ornamento della città, alla quale le vie e gli edifici voluti da Galeazzo II danno nuovo decoro. Lo Studium a sua volta viene «edificato» e illustrato da dottori che Galeazzo ha «procurati da ogni luogo», come fossero pezzi pregiati, perché fungano a loro volta da «fondamento» del nuovo Studium solemne; anche l'aggettivo solemne, che sostituisce il più consueto generale, asseconda l'idea dello sfarzo, in prospettiva appunto evergetica. Del Decembrio resta anche una breve descrizione dell'Università di Praga, con occhio più asciutto: vd. Šmahel 2007, p. 127.

16 Azario 1730, cap. XI, col. $403=1927$, p. 153. Sul "caso" visconteo, volto a utilizzare lo strumento statutario anche per coordinare una «costellazione di realtà territoriali diverse»: Varanini 1986, spec. pp. 703 ss.; importante anche Storti Storchi 2007, spec. pp. 85-113. 
sotto pene discrezionali. È un ordine che contraddice la libertà quasi girovaga dei clerici vagantes. Anche per questa disposizione, lo storico del XIV secolo (e, alla fine del successivo, il Corio che da lui dipende) offre una spiegazione plausibile: Galeazzo II chiama gli studenti a Pavia per riempire un vuoto. ${ }^{17} \mathrm{La}$ città è ancora desolata per l'assedio e anche per la recrudescenza della peste nera, cui accenna persino il diploma imperiale di Carlo IV («odiosa pestilentiae rabies»): $:^{18}$ muoiono sette su dieci, è il macabro conto dell'Azario. Il tempo sembra si sia fermato: le case vuote aspettano nuovi abitatori, i campi attendono chi colga le messi abbondanti. «Hisce consideratis», avendo considerato le caratteristiche ideali della città per accogliere uno Studium, Galeazzo chiama doctores e giovani per riempirla ancora di vita, non lesinando incentivi e senza disdegnare il protezionismo. ${ }^{19}$

Insomma, la lettera del 27 ottobre è incastonata dall'Azario in un'interpretazione degli scopi della fondazione, destinata a imporsi come modello. Abbiamo già avuto modo di accennare a Bernardino Corio, che al principio del ' 500 raccolse in fedele sintesi questa lettura. ${ }^{20} \mathrm{Ma}$ non saranno sfuggiti i molti punti in comune anche con il ritratto del Gymnasium Ticinense steso duecentocinquanta anni dopo dal Costa, da cui abbiamo preso avvio: le favorevoli condizioni ambientali offerte dalla città; $\mathrm{i}$ «doctores sufficientes» del 1361 che corrispondono alla «interpretum praestantia» del 1610; i «privilegia» imperiali (che nel resoconto aggiornato del Costa sono anche quelli papali).

17 Azario 1730, cap. XI, col. $403=1927$, p. 153. Tutta l'interpretazione dell'Azario è ripresa e bene intesa da Corio 1978, I, p. 805: «per le continue guerre essendo la cità vacua de habitatori, Galeazo quanto puotte misse l'animo in ogni studio farla de studenti habundante». Sul Corio, che pubblica nel 1503 la Patria Historia che era stata patrocinata da Ludovico il Moro, vd. Ianziti 1988, pp. 236-238; Meschini 1995; Fiaschi 2012.

18 Vd. il testo edito da Fugazza 2012a, pp. 229-232.

19 Sul divieto di frequentare altri Studia, costantemente ripetuto, vd. Crotti 2012, pp. 244-246.

20 In generale, il racconto dell'Azario fu «molto sfruttato nel secolo XV»: Nada Patrone 1962, p. 742. Per un esempio settecentesco, vd. infra a proposito dell'uso in Antonio Gatti (che la leggeva la cronaca in manoscritto). Un tramite, prima dell'edizione muratoriana del 1730, fu proprio l'opera del Corio (che ne fa menzione esplicita, anche se problematica, ad annum 1385): egli lo conobbe tuttavia attraverso una compilazione conservata a Novara («el Valison»): vd. Cognasso, in Azario 1927, pp. XXVIIIXXIX. 
L'incidenza del modello azariano va anche più in profondità. All'alba del Seicento, il Costa glorificava soprattutto l'antiquitas, l'origine che traguardava il 1361 e rimontava fino a Carlo Magno. Ma già Azario, introducendo e sintetizzando la lettera di Galeazzo II, apriva una vertiginosa retrospettiva: «dominus Galeaz curavit habere universa Studia in Civitate Papiae, in qua antiquitus fuisse dicuntur», ossia «Il signore Galeazzo curò di avere insegnamenti universitari in tutte le materie nella città di Pavia, nella quale si dice che vi fossero nei tempi antichi». ${ }^{21}$ Fin da quando lo Studium fu aperto correva voce che a Pavia vi fosse una tradizione di molto precedente. ${ }^{22}$

Azario, tuttavia, si limitava ad alludere, senza spiegare. Molti, oggi, leggendo le sue parole pensano a Lotario e al Capitolare dell'825, ma è un anacronismo: vedremo più avanti che quello di Lotario è un mito di fondazione "inventato" nel XX secolo; basti dire che il Capitolare da lui emanato a Corte Olona fu edito - e dunque se ne tornò ad avere conoscenza - solo nel $1725 .{ }^{23}$ Un candidato apparentemente più quotato è Carlo Magno. Pensava a lui l'Azario, come più tardi il Costa? Non si può escludere. ${ }^{24}$ Tuttavia, la fondazione carolingia è in concorrenza con un'altra versione, che l'attribuiva ai

21 Azario 1730, cap. XII, col. $406=1927$, pp. 156-157. La frase che segue «et certe de iure bene stat» etc. significa «e certamente, a buon diritto, (lo Studio) sta bene (a Pavia)». È la frase cruciale che segna il passaggio argomentativo; il richiamo ideologico al passato lascia il posto alla spiegazione della bontà della scelta nel presente, basata sulle caratteristiche logistiche della città prese in considerazione da Galeazzo: «Nam ipsa civitas...» etc. «Hisce consideratis curavit habere Doctores...» etc. Contro interpretazioni erronee, vd. infra, nt. 260; per un parallelo, vd. la frase riferita a Galeazzo: «non bene sanus de persona stat»: Id. 1730, cap. XI, col. $403=1927$, p. 153.

22 Secondo Barbieri 2011, p. 25, la frase di Carlo IV «Nos attendentes multiplicia merita probitatis, quibus antedicti Papienses nos et Sacrum Romanum Imperium retroacto iam tempore exquisita diligentia studiis honorarunt» potrebbe alludere alla «precedente tradizione di studi nella città». Non si può escludere; tuttavia, «merita probitatis» esprime un concetto politico e dunque l'impressione è che nella successiva frase relativa - che ne è l'esplicazione - «studiis» abbia valore generico di «sforzi» (e non quello tecnico di «studii»), con valore modale analogo a «exquisita diligentia» cui è collegato asindeticamente.

23 Vd. infra. Nel pubblicarlo, il Muratori 1725, p. 151, nt. 3, faceva notare che era norma solo temporanea, perciò omessa da molti manoscritti. Sulla tradizione manoscritta dei capitolari, vd. analiticamente Bougard 1995, pp. 30-43; 49-52.

24 Lo pensava ad es. il Villa 1782, p. 4, pure fra i più fieri avversari dell'origine carolingia dello Studium (vd. infra). 
Longobardi. Seguiremo ora le tracce di queste due versioni, in un tragitto che ci porterà anche a seguire, di riflesso, alcune tappe della vita dello Studium.

\section{GiAn GALEAZZO VisCONTI E I LONGOBARDI STUDIOSI}

Nei quarant'anni trascorsi dal 1361, lo Studium Papiense plasmò la sua identità, imbastendola intorno a riti (come il conventus di laurea, con le sue gerarchie sancite dalle precedenze), simboli (le insegne dottorali), rappresentazioni di ingresso e di commiato (orazioni inaugurali ed esequie), culto di santi protettori, assetti di insegnamenti, stili didattici. ${ }^{25}$ Nell'iscrizione del 1391 che commemora la dedica di una cappella a santa Caterina nella chiesa di San Tommaso, tutte le corporazioni, i due Collegia dei doctores e le due Universitates degli studenti, si definiscono complessivamente cetus Studii Papiensis, unità che trascende i corpi costitutivi. I proemi degli statuti - stesi dal 1395 al 1409 - sono invece deludenti, per chi vi cercasse tracce di autocoscienza storica, impegnati come sono ad assecondare una logica di contenimento e controllo: del resto, furono composti con l'assenso del dominus Gian Galeazzo e del figlio Filippo Maria. ${ }^{26}$ Tuttavia, di per sé gli statuti, aspirando a essere «regula sempiterna» della vita universitaria, ponevano un'ipoteca di continuità sul futuro e s'inserivano in una linea che aveva già un passato, evocato da «ordinamenta antiqua» che i riformatori dicono di avere tenuto presenti nel redigere i nuovi; un passato che in alcuni casi aveva accumulato ai loro occhi già la lunga densità di una «consuetudine finora osservata» («consuetudo actenus observata»). ${ }^{27}$ Ad aggiornarci sulle condizioni dello Studium all'epoca di Gian Galeazzo Visconti, figlio e successore del fondatore, soccorre un biografo (seppure distante d'oltre un secolo), Paolo Giovio, che a Pavia si

25 Particolarmente importante, sotto l'ultimo profilo, la testimonianza di uno studente resa in calce alle recollectae di Albertino da Salso, Super I Canone Avicenne, nel ms. Parma, Bibl. Palatina, 1041, c. 201v. Il testo è stato edito da Belloni 1982, p. 45.

26 Si distingue l'elogio della Medicina nel proemio dello statuto dei doctores medici, peraltro convenzionale calco dei motivi della contesa fra le arti: Codice diplomatico, I, doc. 183, p. 111. Sul tema dell'autocoscienza professionale dei giuristi, in generale, vd. Meyer-Holz 1989; Brundage 2008; Mazzilli Savini 2011.

27 Statuto dell'Università giurista: Codice diplomatico, I, doc. 46, p. 292, cap. XCV. 
laureò in Filosofia e Medicina nel 1511 e vide Giasone del Maino parlare da pari al re di Francia Luigi XII. ${ }^{28}$ Benché il Giovio non sfugga alla tentazione di presentare il Gymnasium Ticinense come riflesso della personalità del duca benefattore e virtuoso, che «ricreava l'animo co' i ragionamenti de gli uomini dotti, et con lo spesso leggere», la sua descrizione non manca di realismo, a cominciare dalla rinuncia a cercare origini anteriori al 1361:

Haveva condotto con liberali stipendi professori di tutte le scienze, i quali insegnassero alla gioventù nello studio di Pavia fondato da suo padre. [...] Erano allhora huomini singolarissimi dell'ordine de' lettori, i quali hanno lasciato memorie d'ingegno ai posteri, in ragion civile Baldo, e i due Rafaelli, il Fulgoso, e '1 Comasco, ${ }^{29}$ et Signorolo Amadio. In Philosophia Ugo Sanese, et Biagio Pelacane da Parma, di cui ci sono anchora sottilissime questioni nelle cose d'Astronomia, et nella disciplina di Prospettiva, et tre Medici anchora, Marsilio da Santa Sophia, Sillano Negro, et Antonio Vacca, i libri de i quali hoggi si leggono nelle Scuole. Fioriva parimente dell'insegnare i precetti della lingua Greca Emanouello Chrisolora Costantinopolitano. Ma sopra i dottori di tutte le discipline era singolarissimo Pietro Philargo di Candia, interprete delle Sacre lettere, il quale fu poi fatto Papa, et chiamossi Alessandro Quinto. ${ }^{30}$

La schiera ragionata dei doctores è l'indicatore del successo culturale dello Studium fra Tre e Quattrocento, che il Giovio osserva anche nelle sue propaggini attraverso la persistente circolazione, fino ai giorni suoi, delle opere scritte da quei maestri. Il suo schizzo si congiunge a quello dell'Azario (e del Decembrio), ${ }^{31}$ a certificare il successo riscosso dallo Studium Papiense nei suoi primi quattro decenni di vita. $^{32}$

Non aveva bisogno, il Giovio, di nobilitare le origini con richiami

28 Sul Giovio, Price Zimmermann 1995; per l'aneddoto, vd. infra, nt. 106.

29 S'intendono Raffaele Fulgosio e Raffaele Raimondi, su cui Necchi 1994, pp. $215-222$

30 Giovio 1549, c. 99r. L'effettivo insegnamento del Filarge è discusso: vd. Corbellini 1915, pp. 355-365.

31 Vd. supra, nt. 15.

32 Queste testimonianze rendono ancora più probabile che il momentaneo trasferimento a Piacenza (su cui Fugazza 2012b), fra la fine del 1398 e il 1402, non avesse a che fare con una crisi (bensì con un'epidemia) o che, se crisi vi fu, dipese semmai dal successo, con conseguenti problemi di compatibilità con la città. 
all'antichità, perché il suo intento era di descrivere le imprese dei Visconti (o il loro carattere attraverso le imprese), in coerenza con il suo credo storico:

Coloro che ambitiosamente s'ingegnano derivare l'antichità della nobilissima famiglia de Visconti dall'altissima origine de Cesari Romani, et da i Re Longobardi per lunga successione, pare che quasi la involgano in favolosi principij. Ma io seguirò cose più fresche et più chiare $[\ldots] .^{33}$

A involgersi in «favolosi principij», in quelle genealogie incredibili da cui il Giovio dichiarava di astenersi, erano stati tuttavia fin dalla prima metà del Trecento gli stessi Visconti, ovviamente allo scopo di accreditare la legittimità del loro potere..$^{34}$ Emblematico, sotto questo profilo, è il discorso tenuto da Pietro da Castelletto, frate eremitano di Sant'Agostino in Pavia, alle esequie di Gian Galeazzo, morto inopinatamente il 3 settembre $1402::^{35}$ parte dell'orazione è una litania onomastica che lega il defunto ai re longobardi e risale fino al capostipite Anglo figlio di Ascanio figlio di Enea. Il preteso lignaggio longobardo dei Visconti si coordinava funzionalmente con il ripristino della memoria di Pavia come capitale altomedievale del regno: si tratta di un'elaborazione ormai ben chiarita sul piano generale (il «sogno regio dei Visconti»), ${ }^{36}$ che qui occorre approfondire con riguardo specifico allo Studium.

Documento privilegiato di quest'operazione politico-ideologica è il Codice dal Verme, una raccolta di testi prodotta, nel suo principale e più antico nucleo, da ambienti prossimi alla cancelleria di Gian Galeazzo, il duca che «con spaventosa grandezza s'inalzò sopra gli altri Prencipi d'Italia» e che s'era dotato di uffici che il Giovio giudicava «fondati con maraviglioso ordine» su un professionale uso della scrittura e dell'archiviazione. ${ }^{37}$ Gli opuscoli di questo centone composti da

33 Giovio 1549, c. 6r. La prima edizione, in latino, era apparsa sempre nel 1549 a Parigi; il termine latino che impiega è Gymnasium Ticinense.

34 Per le genealogie incredibili il riferimento classico è a Bizzocchi 2009. Altra bibliografia supra, nt. 9.

35 Majocchi 2008, pp. 217-218; il Sermo è edito in Pietro da Castelletto 1730, coll. 1038-1050: non vi sono accenni allo Studium.

36 Il riferimento è a Settia 1997, pp. 13-15; importante anche Majocchi 2008, spec. pp. 189-225.

37 Giovio 1549, risp. cc. 88r; 99v-100r. 
«mano cancelleresca dell'ultimo decennio del XIV secolo, integrate da altre mani (con minori caratteristiche notarili) del primo XV», sono legati dal filo dell'esaltazione di Pavia - delle sue origini, della sua religiosità e del ruolo di capitale altomedievale e soprattutto longobarda che si intreccia con l'encomio di Gian Galeazzo. ${ }^{38}$

Fra gli opuscoli del codice spicca il testo intitolato Instituta regalia et ministeria camere regum Longobardorum et honorancie civitatis Papie, che descrive l'organizzazione dei funzionari e le loro competenze, specialmente per quanto riguarda l'esercizio delle regalie, ossia i diritti regi alla percezione di tributi e dazi e alla monetazione. ${ }^{39}$ Secondo l'ipotesi più accreditata, gli Instituta furono scritti fra il 1000 e il 1020, probabilmente in varie tappe e servendosi di modelli più antichi, per mano di Gisulfo, già attivo come magister camerae fra il 991 e il 1004.

Nel Codice dal Verme, gli Instituta sono aperti da un prologo in lode della città, le Honorantie civitatis Papie. A partire dalla condizione di seconda Roma («Roma nominat Papiam et appellat filiam suam»), la città è presentata come luogo di incoronazione regia, sede di «regale palatium» al quale si devono recare «omnes principes Italie», dotato di «comites palatii», «missi regis» e «iudices»; insomma «Papia debet habere regem $\gg^{40}$

In quest'inno alla città sotto $\mathrm{i}$ «reges Longobardorum», si legge:

Omnes insuper iudices Italie debent quaestiones per sententiam iudicare, quia ex omnibus civitatibus Italie veniebant ad Generale Studium huius alme civitatis Papie studere in iure civili et leges adiscere; et maiores magisque honorati fuere iudices Papie

ossia: «inoltre tutti i giudici d'Italia devono giudicare le controversie mediante sentenza, poiché da tutte le città d'Italia venivano al generale Studium di questa alma città di Pavia a studiare in Diritto civile e imparare le leggi; e i giudici di Pavia furono maggiori e più onorati». ${ }^{41}$

38 Descrizione del contenuto del Codice dal Verme in Brühl - Violante 1983, pp. 1-6; Majocchi 2008, pp. 233-307, con trascrizione dei 19 testi databili fino alla metà del Quattrocento (ivi, p. 212 la citazione).

39 Ed. Brühl - Violante 1983, pp. 16-27; cfr. Majocchi 2008, pp. 278-283.

40 Ed. Brühl - Violante 1983, p. 17, rr. 1-27.

41 Ed. Brühl - Violante 1983, p. 17, rr. 19-22, con diversa punteggiatura: il testo instaura un nesso causale fra il fatto che a Pavia vi fosse un insegnamento e il ruolo dei 
Se la frase fosse stata scritta da Gisulfo, intorno all'anno Mille, sarebbe prova certa della presenza a Pavia dello Studium ben prima della fondazione nel 1361. In questo senso, ad esempio, queste parole furono intese nel 1587 da Alessandro da Rho, professore di Diritto, in una delle rare menzioni delle Honorantie durante il percorso carsico che le ha quasi completamente nascoste prima della trascrizione del Moiraghi alla fine del XIX secolo. ${ }^{42}$ Si ritiene oggi, giustamente, che questo brano - insieme ad altri - sia un'interpolazione, un'aggiunta posteriore alla fondazione del generale Studium nel 1361, effettuata con ogni probabilità alla fine del XIV secolo, più o meno nello stesso momento in cui il testo veniva copiato nel Codice dal Verme. ${ }^{43}$ Le Honorantie perdono così valore come prova dell'origine altomedievale. Esse tuttavia diventano - non mi pare sia stato notato - prezioso documento della coscienza storica del loro estensore, poiché questa interpolazione è la prima traccia della versione longobarda del mito di fondazione (ed è al tempo stesso testimonianza più generale del valore che si annetteva all'antiquitas per legittimare lo Studium).44

Questa interpolazione, che legava ai reges Longobardorum anche lo Studium - non solo la città e i Visconti - è certamente consona all'ideologia sviluppata intorno alla cancelleria di Gian Galeazzo Visconti. Non è possibile andare oltre, affermare cioè se chi ha scritto quelle righe sia stato anche l'autore (o comunque il portavoce) di questo mito di fondazione, sorto perciò alla fine del Trecento, oppure se egli abbia approfittato della cornice offerta dagli Instituta e dalle Honorantie per dare veste autorevole a un'idea che già circolava. Si ritorna così a qualche decennio prima, alla cronaca di Pietro Azario: «in qua antiquitus fuisse dicitur». Era forse la presenza dello Studium all'epoca dei Longobardi la diceria che circolava al suo tempo?

«iudices Italie», anche se non è possibile precisarlo. Nel seguito, si dice parallelamente «ex omnibus civitatibus Italie extiterunt episcopi Papie» (rr. 22-23).

42 Vd. infra, nt. 137.

43 Brühl - Violante 1983, p. 77, propendono per l'idea che l'elogio con cui si aprono gli Instituta ampliasse uno testo antico; tuttavia, ritengono che l'intera frase relativa allo Studium sia aggiunta di fine XIV secolo.

44 Inversamente, l'aggiunta significa che lo Studium era visto come elemento nobilitante della città, da retroproiettare. 
Qui - senza avanzare una risposta - vale la pena di notare che il motivo della dignità regia di Pavia longobarda, quando lo si colleghi alla fondazione del generale Studium, rivela una particolare densità, un valore quasi giuridico. ${ }^{45}$ Una costituzione di Giustiniano del 533 d.C. (ovviamente considerata vigente dai medievali) stabiliva che l'insegnamento del Diritto potesse svolgersi esclusivamente nelle città regie, «in regiis urbibus» (c. Omnem, $\$ 7$ ). Pene severe erano comminate a chi insegnasse altrove (con l'unica eccezione di Berito). Naturalmente, questa norma creava un grave problema agli interpreti medievali, che dovevano giustificare la fioritura di sedi altrove, da Bologna in poi. Le strade percorse per aggirare il divieto furono molte: un apposito privilegio imperiale o papale, oppure una consuetudine lunghissima («cum talis consuetudo sit similis privilegio»).46

Un'altra strada - quella che ci interessa - fu di appigliarsi alla locuzione «in regiis urbibus», con cui Giustiniano intendeva ovviamente Roma e Costantinopoli, capitali della sua regalità, ma che per i medievali poteva estendersi anche ad «altre città fondate da re e principi». ${ }^{47}$ Pavia, dicendosi urbs regia, rafforzava perciò la propria pretesa di avere un generale Studium, perché il privilegio imperiale - che Galeazzo II domandava a Carlo IV - avrebbe confermato ciò che, in un certo senso, le spettava già in virtù di questa sua natura. ${ }^{48}$ Può dunque

45 Vale la pena di precisare che la dignità regia della città è aspetto diverso dalla presenza dello Studium; possono tuttavia essere collegati, come nella spiegazione che segue.

46 Così la postilla dell'imolese Alessandro Tartagni (1424-1477) al commento di Bartolo da Sassoferrato (1314-1357), ad c. Omnem, $\$ 7$, gl. Haec autem tria (ed. 1537, c. 7 r), da cui anche la successiva citazione. L'assimilazione fra consuetudine e privilegio contiene in nuce l'equiparazione fra storia e diritto nella mentalità medievale. Per tutti Nardi 1992, pp. 47-78; Id. 2007, pp. 49-59.

47 Già la glossa di Accursio alla c. Omnem $\$ 7$, all'inizio del XIII secolo, sosteneva che Bologna fosse stata riedificata da Teodosio I: per tutti Nardi 1992, p. 49.

48 Naturalmente, oltre che per dare certezza al titolo giuridico, l'autorizzazione dell'imperatore (o del papa) era comunque desiderabile per dare al dottorato il valore di licentia ubique docendi e per conferire uno status privilegiato a docenti e studenti, ponendoli sotto la propria tutela politica. Sono obiettivi ben presenti all'Azario 1730, cap. XII, col. $406=1927$, pp. 156-157: Galeazzo «curavit habere privilegia et facultatem conventandi». Vd. Frova 2012, pp. 18-28 e Crotti 2012, pp. 237-246, che sottolinea maggiormente le motivazioni proprie di Carlo IV nel perseguire una politica di moltiplicazione degli Studia in Europa. 
essere plausibile che già nella richiesta inviata a Carlo IV si alludesse all'antica qualità regia dell'urbs e alla presenza di uno Studium.

Occorre fermarsi a quest'ipotesi per quanto riguarda Galeazzo II, avendo in ogni caso acquisito come punto fermo l'esistenza di questa idea al più tardi qualche decennio dopo il 1361, al volgere del Trecento, nel Codice dal Verme.

Prima di abbandonare il testo delle Honorantie conviene rilevare - per cogliervi il riflesso delle idee viscontee - che il mito di fondazione vi è inserito in modo tale da glorificare sì lo Studium in virtù della sua antichità, ma anche da additarne il ruolo funzionale all'istruzione dei giudici, a servizio di una corretta amministrazione della giustizia (che corrisponde in fondo allo scopo che Azario attribuiva alla reale fondazione dello Studium da parte di Galeazzo II).

Con la morte di Gian Galeazzo, nel 1402, si apre un decennio di grande incertezza, che l'Università - che ci è apparsa fiorente nel quadro retrospettivo di Giovio - patì fino al collasso. Il 16 maggio 1412 Giovanni Maria Visconti veniva assassinato a Milano; poche ore dopo, a quanto pare, Facino Cane moriva a Pavia, la città che due anni prima aveva saccheggiato: duca diventava Filippo Maria, il più giovane dei figli di Gian Galeazzo. Da subito, il duca rivolse il suo pensiero allo Studium che «era rimasto sospeso ed è sospeso, trascorsi parecchi anni, mentre si moltiplicavano i dissidi delle guerre, da cui questa patria Lombardia - che pena! - è stata a lungo oppressa e vessata».49 Ora anelava a un rilancio, che sarebbe stato insieme rilancio della città («ut [...] civitas haec restauretur pariter et augmentetur»). Era tempo di annunciarlo, diffondendo agli ufficiali una lettera che ricorda nella sua tessitura quella con cui Galeazzo II aveva dato il primo annuncio nel 1361 (o 1362).

Innanzitutto, il duca assicura che Pavia è ricca di vitto («frugum nunc et omnium victualium satis ubertate repleta»), sufficiente per «tutti quelli - Lombardi, Italiani e tutti quanti anche al di qua e al di là delle Alpi - che desiderano ottenere la conoscenza» («omnibus Logombardis et Italicis etiamque tam Citramontanis quam Ultramontanis, scienciam adipisci cupientibus»). A ciascuno, anche non suddito del ducato, viene garantito di accedere alla città «con i suoi libri, robe e beni, e di rimanerci a suo piacimento, liberamente e impu-

49 Codice diplomatico, II.1, doc. 195, p. 124, da cui anche le citazioni successive. Per la probabile datazione all'ottobre 1412, vd. nt. seguente. 
nemente». Questi motivi saranno ben messi in evidenza dagli ufficiali che nei giorni seguenti diffonderanno l'annuncio ducale, in forma di proclama, a Tortona, Novara, Alessandria e Asti. Nella sua lettera, tuttavia, accanto a queste notizie pratiche che trovarono la strada del pubblico, il duca aveva esibito anche un erudito richiamo all'alta antichità dello Studio (che i podestà non riprodussero):

infatti nella predetta città regia [di Pavia] lo Studio generale fiorì ai tempi di re Liutprando - figlio del re Asprando - che morì a Pavia nell'anno del Signore 741 e giace nella chiesa di Sant'Agostino, che eresse sotto il titolo di San Pietro in Ciel d'Oro; e di Desiderio, ultimo re dei Longobardi, che regnò a Pavia per ventisei anni, dall'anno del Signore 754; re Longobardi che per la maggior parte sono sepolti qui nelle chiese da loro stessi edificate. Trascorsi molti anni lo Studio generale fiorì nella predetta città iniziando da Berengario primo, che imperò dall'anno del Signore 914 ecc., unico in Italia, fino a Ottone III, figlio di Ottone II, nell'anno del Signore 972, come è più ampiamente descritto ne Gli Istituti regii, e gli uffici dei Longobardi e Le Onoranze della città di Pavia. ${ }^{50}$

La citazione esplicita non lascia dubbi: nella cancelleria ducale circolava il testo degli Instituta e delle connesse Honorantie e il figlio di Gian Galeazzo si sentiva ancora in sintonia con la valorizzazione longobarda di cui il testo era portatore. ${ }^{51}$

50 Ivi: «Studium enim generale floruit in dicta regia civitate temporibus Regum Liutprandi, filii Regis Asprandi, qui obiit in Papia anno Domini DCCXLJ, et iacet in Ecclesia Sancti Augustini, quam sub vocabulo Sancti Petri in celo aureo fabricavit; et Desiderii, ultimi regis Logombardorum, qui regnavit in Papia annis XXVJ, anno Domini DCCLIIIJ; qui quidem Reges Logombardorum iacent, pro maiori parte, in ecclesiis hic per eos constructis. Etiam revolutis plurimis annis, Studium generale floruit in dicta civitate, incipiendo a Berengario primo, qui imperavit anno Domini DCCCCXIII etc. solus in Italia, usque ad Octonem tercium, Octoni secundi filium, anno Domini DCCCCLXXIJ, prout in Institutis Regalibus et Ministeriis Regum Logombardorum et honoranciis civitatis sepedictae lacius continetur». Per le città in cui fu diffuso il proclama, vd. Codice diplomatico, II.1, doc. 196, p. 125 (12 ottobre 1412), che garantisce la datazione di poco anteriore della lettera ducale. Un accenno alla presenza dello studio «priscis temporibus» dei re longobardi è già nelle credenziali degli ambasciatori inviati dal Comune di Pavia al duca il 25 agosto 1412 (ivi, doc. 189, p. 120); è possibile dipenda dalle Honorantie.

51 Hofmeister, in MGH SS 30,2 (1934), p. 1445; Brühl - Violante 1983, pp. 7 e 85, nt. 134; Majocchi 2008, p. 220, dove è anche discusso se la cancelleria disponesse, com'è probabile, proprio del Codice dal Verme. 
Se nell'interpolazione eseguita qualche anno prima quella frase aveva un valore solo ideologico-politico, adesso, incorporata in una lettera ducale, l'origine longobarda diventa con Filippo Maria la versione per così dire ufficiale dell'origo dello Studium. Un uso della storia in funzione di legittimazione che, come s'è accennato, pareva peraltro più facilmente spendibile all'interno del circuito dei funzionari che da esibire al largo pubblico.

Un punto resta da approfondire. L'affermazione di Filippo Maria, che lo Studium fiorisse già al tempo di Liutprando e di Desiderio, non è ricavabile dal testo degli Instituta e delle Honorantie, dove i due re non sono citati. Le Honorantie - nella frase che, come sappiamo, fu aggiunta per interpolazione al tempo di Gian Galeazzo - affermano senza precisazioni cronologiche che «ex omnibus civitatibus Italie veniebant ad Generale Studium huius alme civitatis Papie studere in iure civili et leges adiscere». Si deve pensare che Filippo Maria fosse invece a conoscenza di una tradizione che faceva risalire più precisamente a Liutprando la fondazione dello Studium?

In realtà, se seguiamo la pista dei testi, arriviamo alla sicura conclusione che Filippo Maria - o meglio i suoi segretari - abbiano compiuto una semplice operazione di assemblaggio, attingendo ad altri opuscoli raccolti nel medesimo manoscritto (il Codice dal Verme). È fin troppo evidente che così sia avvenuto per i nomi dei due sovrani postcarolingi, che sono tratti dal catalogo che nel codice segue immediatamente gli Instituta e che si apre appunto con Berengario I e si chiude con Ottone III; anche le coincidenze testuali (per esempio il «solus in Italia» riferito a Berengario) garantiscono la derivazione. ${ }^{52}$ I nomi dei due re longobardi furono ricavati, a loro volta, da due altri elenchi che nel Codice dal Verme precedono gli Instituta, ossia la cronaca dei re longobardi che inizia con il lemma «Infrascripti reges Longobardorum» nonché il «Sumarium regum Longobardorum, qui iacent in Papia in ecclesiis infrascriptis». ${ }^{53} \mathrm{Il}$ primo elenco offriva a

52 MGH SS 30,2 (1934), pp. 1459-1460; vd. anche Hofmeister, ivi, p. 1445, ntt. 2 e 3; Majocchi 2008, pp. 284-285.

$53 \mathrm{Vd}$. la trascrizione dei due testi (risp. ms. dal Verme, cc. $8 r-12 r$; c. 13r) in Majocchi 2008, risp. pp. 253-259; 262. Se è esatta la mia ipotesi, si ha conferma che chi scrisse la lettera di Filippo Maria del 1412 aveva sotto gli occhi il Codice dal Verme o almeno un manoscritto che conteneva i medesimi opuscoli (e non leggeva invece una isolata edizione delle Honorantie). 
Filippo Maria le notizie relative alle sepolture e alla costruzione della chiesa di San Pietro in Ciel d'Oro; il secondo i dati cronologici. ${ }^{54}$

Svanisce, a mio parere, la possibile illazione che la cancelleria di Filippo Maria disponesse di (altre) notizie su un'origine longobarda. ${ }^{55}$ Nel redigere l'annuncio di riapertura dello Studium, s'ingegnò per dare contorni cronologici alla notizia delle Honorantie, attingendo ai cataloghi contenuti nello stesso manoscritto; il catalogo delle sepolture dei re longobardi si prestava inoltre a un'aggiuntiva valorizzazione della nobiltà politica e religiosa di Pavia. Anche il silenzio sulla fondazione carolingia - e soprattutto l'apparente dinamismo narrativo d'una fondazione longobarda, d'un intervallo e di una ripresa da Berengario a Ottone - sono solo il riflesso della combinazione dei due cataloghi.

Nei decenni che seguirono, e per tutto il Quattrocento, furono molte e varie le testimonianze del revival longobardo e della «riscoperta del ruolo di capitale del regno promossa nell'età di Gian Galeazzo».56 Non sembra, tuttavia, che il nesso stabilito fra Liutprando e lo Studium abbia avuto particolare fortuna ${ }^{57}$ almeno, non m'è riuscito di trovarne altra testimonianza fino al 1553, in un'orazione tenuta da Aldigieri Cornazzano quando ricevette la laurea in Giurisprudenza (e riedita nel secolo successivo da un discendente), contesto nel quale l'antiquitas serve a esaltare lo Studium e, implicitamente, il laureato. La citazione che ne fa il Cornazzano nella sua orazione di laurea attira l'attenzione per un'altra ragione, perché include nella preistoria favolosa del Gymnasium, dopo l'exordium attribuito al re longobardo Grimoaldo, anche un ampliamento dell'Università sotto Carlo Magno. ${ }^{58}$

54 Anche nella lettera, come nel catalogo (c. 12r; Majocchi 2008, p. 259) gli anni del regno di Desiderio sono per errore XXVI, invece di XVI.

55 A meno di leggerla nel criptico verso del ritmo celebrativo di Liutprando: «[...] Iohannes Galeaz. Is illum regem pium [ossia Liutprando] imitatur. Probat eius studium, quo et transformatur in Christi obsequium, ut factis affatur»; vd. la trascrizione del testo (ms. Dal Verme, c. 14r) in Majocchi 2008, p. 263.

56 Così Majocchi 2008, p. 220; ivi, pp. 219-225 le testimonianze.

57 Interessante, purtroppo generica, l'affermazione «Tenuisti [scil. Papia] perpetuo famosissimum Studium omnis sophie», espressa nel 1418 da Giuseppe Brivio, canonico ordinario del Capitolo del Duomo di Milano, in occasione della visita di Papa Martino V: Codice diplomatico, II.1, doc. 257, p. 175.

58 Cornazzano 1643, pp. [1-2]): «Agitur, Viri Papienses, millesimus prope annus, cum Vestrum hoc Gymnasium a clarae memoriae Longobardorum, et Italiae Rege, Grimoaldo, sumptis exordijs, longa vero post intervalla ab Augustissimo Carolo 
Quando scriveva il Cornazzano, a metà Cinquecento, accanto alla versione longobarda aveva in effetti preso quota, e s'era anzi rapidamente imposto, un diverso racconto di fondazione, che aveva il pregio di unire il prestigio di Carlo Magno alla sincronia fra la scuola di Pavia e quella di Parigi.

\section{I mercanti di sapienza: Carlo Magno, Parigi e Pavia}

Tutto nasce da una pagina di uno dei più antichi biografi di Carlo Magno, Notker Balbulus (†912). Notker apparteneva all'abbazia benedettina di San Gallo (Fürstabtei Sankt Gallen, oggi nell'omonimo cantone svizzero), fondazione carolingia della prima metà dell'VIII secolo, e scriveva su committenza dell'imperatore Carlo il Grosso, intorno all'884-887..$^{59}$ Proprio in apertura della sua opera, ai capitoli 1 e $2,^{60}$ Notker racconta di due monaci irlandesi («duos Scottos de Hibernia») venuti a visitare le coste della Gallia in compagnia di commercianti britannici; i due erano senza rivali nella conoscenza delle lettere sacre e profane, in un'epoca in cui cultura e (di conseguenza) fede ortodossa erano pressoché dimenticate nel regno di Carlo Magno («cum [...] studia litterarum ubique essent in oblivione ideoque verae deitatis cultura teperet»: da notare il nesso di consequenzialità). I due irlandesi, privi di merce da vendere, adottano una tecnica di comunicazione efficace. Al mercato, lanciano questo richiamo: «Se qualcuno è desideroso di sapienza, venga da noi e l'avrà; è in vendita presso di noi» («Qui cum nihil ostenderent venale, ad convenientes emendi gratia turbas clamare solebant: "Si quis sapientiae cupidus est, veniat ad nos et accipiat eam; nam venalis est apud nos"»). I due monaci sono consapevoli che il pub-

Magno auctum, eo demum vita functo, ac tota Francorum stirpe poenitus extincta [...] a Berengario deinceps III eius ex Longobardico sanguine nominis restitutum, et a Vicecomite Joanne Galeacio, Principe Regibus ipsis aequiparando, maxime fuit insignitum: ut propterea inter omnem Italica Gymnasia, Vestrum, Papienses, iure quidem claro, cunctis superemineat». La menzione di Grimoaldo (il re che ristabilì definitivamente Pavia come capitale) fa pensare che il Cornazzano attingesse a una versione diversa da quella delle Honorantie e della lettera di Filippo Maria Visconti del 1412.

59 Vd. Häfele, in Gesta Karoli Magni Imperatoris, MGH SS rer. Germ. n.s. 12 (1959), spec. pp. VII-XXVII; vd. anche Tremp 2009, pp. 15-40.

60 MGH SS rer. Germ. n.s. 12 (1959), pp. 1-4. 
blico non apprezza quel che può avere gratuitamente, ma solo ciò che occorre pagare; d'altra parte, confidano che il messaggio inconsueto attirerà comunque l'attenzione («Quam tamen iccirco venalem se habere professi sunt, quia populum non gratuita sed venalia mercari viderunt, ut sic vel sapientiae sicut caeteris rebus coemendis eos incitarent vel, sicut sequentia comprobant, per tale praeconium in admirationem verterent et stuporem»).

La fama dei due irlandesi mercanti di sapienza arrivò, in effetti, alle orecchie di Carlo Magno («semper amatoris et cupidissimi sapientiae»), che li convocò e poi li trattenne offrendo loro quel che avevano chiesto, cioè ben poco per sé - un modico sostentamento - ma aule e soprattutto allievi meritevoli («Qui cum inquisisset ab illis, quid pro ipsa peterent, responderunt: "Loca tantum oportuna et animos ingeniosos et, sine quibus peregrinatio transigi non potest, alimenta et quibus tegamur"»): condizioni che fanno dei due monaci il simbolo, in ogni tempo, di un disinteressato amore per la trasmissione della cultura. Il re fu però ben presto assorbito da incombenze militari, perciò stabilì uno degli intellettuali - di nome Clemens ${ }^{61}$ - in Gallia, affidandogli l'istruzione di un gran numero di giovani, di tutti i ceti sociali. Il secondo - il cui nome è sfortunatamente caduto vittima di un guasto nella tradizione manoscritta - fu invece inviato da Carlo Magno a Pavia, a capo del monastero di Sant'Agostino a Ticinum, perché qui si potessero recare a lui quanti desiderassero istruirsi ${ }^{62}$ («alterum vero nomine $<\ldots\rangle$ in Italiam direxit, cui et monasterium Sancti Augustini iuxta Ticinensem urbem delegavit, ut illuc ad eum qui voluissent ad discendum congregari potuissent»).

Grazie al memorabile racconto dei due monaci irlandesi disinteressati mercanti di sapienza arruolati da Carlo Magno, Ticinum-Pavia faceva dunque irruzione nell'orizzonte europeo dei centri d'alta cultura: un racconto - andrà sempre tenuto presente, quand'anche se ne rilevi il carattere aneddotico e apologetico (secondo il monaco sangallense, Carlo Magno risollevò l'istruzione per risvegliare «verae dei-

61 Forse da identificare con il Clemens Scotus autore di una grammatica in forma di dialogo, su cui Lexicon des Mittel Alters, II, 1983, c. 2149 (G. Silagi).

62 Parte dei mss. reca la lezione «Albinum», che però pare ricavato dalla successiva menzione di questo personaggio al cap. 2: vd. l'apparato critico in MGH SS rer. Germ.n.s. 12 (1959), p. 3, r. 17. 
tatis cultura») e se ne constatino le posteriori strumentalizzazioni che difficilmente avrebbe potuto formarsi all'altezza del IX secolo d.C. se il monastero di Sant'Agostino «apud Ticinensem urbem» non avesse avuto già allora un saliente rilievo culturale. ${ }^{63}$ Con l'ingresso in scena dei monaci e di Carlo Magno, anche il nostro racconto sulle origini dello Studium si deve trasferire ora in un orizzonte più vasto di quello locale o lombardo in cui s'è fin qui svolto, da Azario a Filippo Maria Visconti: simbolo della funzione che l'Università ha spesso svolto nel collegare Pavia - ben al di là del suo ruolo politico - alla storia europea.

Il racconto di Notker fu raccolto fra i primi dal domenicano francese Vincenzo di Beauvais (ca. 1190-1264) nella sua cronaca universale, lo Speculum bistoriale, in un capitolo dedicato ad Alcuino, presentato come colui che trasferì il «sapientiae studium» da Roma a Parigi (come in precedenza era passato dalla Grecia), dove fondò lo Studium insieme ad altri tre fundatores allievi come lui di Beda, ossia Rabanus, Claudius e Iohannes Scotus. ${ }^{64}$ E dopo avere offerto questa notizia che Vincenzo di Beauvais riporta per intero la pagina di Notker (il cui nome non compare), affermando di trovarla in una (perduta) cronaca della città di Arles. ${ }^{65} \mathrm{La}$ connessione fra i due monaci irlandesi (Clemens lasciato in Gallia e l'anonimo inviato a Pavia) e i quattro fundatores è operata nel disegno di Vincenzo di Beauvais grazie a un minimo ritocco della fonte. ${ }^{66}$ Notker proseguiva affermando che Albino («discipulus doctissimi Bedae»), cioè Alcuino, venuto a sapere della sensibilità con cui Carlo Magno aveva accolto i due monaci, si era recato presso di lui (con

63 Così, equanimemente, un pur fiero avversario dell'origine carolingia come il Villa 1782, p. 36.

64 Speculum bistoriale, XXIII, cap. 173 (ed. Strassburg, ca. 1473, III, c. 201v). Sul Bellovacensis, vd. Paulmier-Foucart 2004; sulla sua circolazione e fortuna fino all'età contemporanea vd. Schneider 1997, pp. 21-46.

65 «In Cronicis metropolis Arelatensis». La pagina che risale a Notker - e dunque la menzione di Pavia - è omessa dal Chronicon Pontificum et Imperatorum di Martino Polono (Oppaviensis), morto nel 1278 (in MGH SS 20,1 [1872], pp. 426-427), che per quanto riguarda il ruolo di Alcuino coincide invece con Vincenzo di Beauvais (che anzi menziona fra le proprie fonti). Per la genesi e la fortuna della notizia relativa ai quattro fundatores dello Studium parigino, vd. D'Onofrio 2009, pp. 439-441, che mette in luce come si basi su di essa l'idea moderna di una continuità istituzionale fra la scuola palatina di Carlo Magno e la Sorbona.

66 Sempre che non si trovasse già nella perduta cronaca di Arles. 
il suo incoraggiamento, «concessa navi»); Vincenzo di Beauvais aggiunge - non mi pare sia stato notato - che Alcuino vi si recò «cum sociis», insieme ai compagni, insomma gli altri tre fundatores. In questo modo, le due tradizioni sono raccordate. ${ }^{67}$

Gli itinerari attraverso cui si diffuse la pagina di Notker - direttamente o tramite la mediazione dell'enciclopedia medievale di Vincenzo di Beauvais o di altri lettori - sono di una tortuosità che non si può qui tentare di seguire. ${ }^{68}$ Un nome che s'incontra in una testimonianza alle soglie del Quattrocento, più o meno coeva al momento in cui nella corte di Gian Galeazzo si diffondeva (e forse si creava) la tradizione relativa all'origine longobarda dello Studium Papiense, ci apre tuttavia un prezioso spiraglio.

La testimonianza è di Francesco Zabarella, nel commento alla Clementina De magistris $(\mathrm{V}, 1,1)$, ossia il decreto di Papa Clemente V risalente al concilio di Vienne del 1311, che istituiva insegnamenti di lingua ebraica, araba e caldea nella curia romana e «in Parisiensi et Oxoniensi, Bononiensi et Salamantino studiis», con l'obiettivo di formare cristiani capaci di propagare la fede in Oriente. La menzione di queste sedi universitarie diede occasione, ai giuristi che commentarono la Clementina, per apporvi notizie storiche. Ė quanto fece appunto il cardinale Zabarella (1360-1417), canonista laureato a Padova dove fu a lungo professore (oltre a svolgere un ruolo di primo piano nella soluzione dello scisma d'Occidente), che glossando la costituzione e incontrandovi appunto lo Studium parigino si propose di stabilire «quomodo incepit et quando» (e analogamente fece per gli altri

67 L'aggiunta è lasciata cadere da Heinrich von Herford (ca. 1300-1370), nel Liber de rebus memorabilioribus sive Chronicon, che pure attinge esplicitamente a Vincenzo di Beauvais (ed. Potthast 1859, p. 34). A un altro versante della «complicata storia di intrecci tra la leggenda dei mercanti di sapienza e quella dei fondatori di Parigi» fa riferimento D’Onofrio 2009, p. 441, nt. 6, che individua Gaguin († 1501) tra «i primi testimoni moderni della fusione tra i due filoni», anche se è soprattutto a partire dalle «informazioni parallele» di Trithème $(\dagger 1516)$ e di Bale $(\dagger 1563)$ (riportati da Brennan 1986, risp. p. 449, nr. 30 e pp. 450-453, nr. 32) che l'identità dei due personaggi citati da Notker si definisce e si iscrive nel gruppo dei fundatores (Claudio Clemente e Giovanni).

68 Sulla circolazione dell'opera vd. Häfele, in Gesta Karoli Magni Imperatoris, MGH SS rer. Germ.n.s. 12 (1959), pp. XXIII-XXVII; D’Onofrio 2009, p. 440, nt. 4. Un'analisi della circolazione potrebbe contribuire a chiarire se le voci cui allude Pietro Azario si riferissero o meno alla fondazione carolingia. 
Studia menzionati: fra i quali come sappiamo non c'è Pavia) ${ }^{69} \mathrm{La}$ risposta che diede lo Zabarella è precisamente quella già approntata da Vincenzo di Beauvais, ossia il racconto dei quattro fundatores (il quarto incongruamente qui chiamato Hestorus) unito a quello dei due monaci irlandesi mercanti di sapienza, uno dei quali inviato a Pavia.

Il canonista patavino stendeva questo commento fra la fine del '300 e l'inizio del '400. Qual era la sua fonte? Piuttosto che leggere Vincenzo di Beauvais, Zabarella trascriveva pressoché alla lettera il commento di Stephanus Hugoneti (Hugonet): un commento che, dopo avere riscosso ampio successo nelle generazioni immediatamente successive, s'inabissò ed è da pochi decenni tornato alla luce in un manoscritto conservato a Philadelphia (Penn.).

Quest'aggancio - in un percorso di testi indispensabile per seguire le idee - ci aiuta a stendere un filo quasi sull'intero arco del Trecento. L'Hugoneti era un provenzale (forse di Narbonne) che dal $1330 \mathrm{fu}$ vescovo di Bologna fino alla morte nel $1332^{70}$ ed ebbe un ruolo attivo nelle vicende politiche della Lombardia, come segretario del legato papale, Bertrand du Poujet (in questa qualità pronunciò anzi la scomunica di Matteo Visconti e dei figli nel 1320). Scritta fra il 1324 e il 1330, mentre era a Parma, la glossa dell'Hugoneti alla clementina De magistris - dove a proposito di Parigi riporta integralmente il racconto dei mercanti compreso l'invio di uno di essi a Pavia - poté dunque essere veicolo di diffusione della notizia, prima della fondazione dello Studium generale nel $1361 .^{71}$

69 Ad Cle. 5.1 .1 v. Inter, q. 5: consulto l'ed. Zabarella 1602, cc. 166v-167r. Fu verosimilmente composta fra la metà degli anni ' 80 e il 1402; sullo Zabarella, Girgensohn 1993, pp. 232-277; p. 250 per la data.

70 La sua glossa si legge nel ms. Philadelphia, Univ. Penn. Library, Codex 643, c. $53 r$-v. L'Hugoneti (a differenza di Zabarella) riporta i nomi dei quattro fundatores dello Studio parigino così come nella tradizione di Vincenzo di Beauvais (e menziona anch'egli la cronaca di Arles come fonte del racconto dei monaci mercanti di sapienza). Da segnalare anche l'attualizzazione toponomastica apposta dall'Hugoneti («apud Ticinensem urbem quae hodie appellatur Papia»), che manca in Vincenzo di Beauvais (e poi ad esempio in sant'Antonino), ma è ripresa sinteticamente da Zabarella (che omette invece l'originario «Ticinensem urbem»). Sull'Apparatus super constitutionibus Concilii Viennensis e sull'Hugoneti - in corrispondenza con papa Giovanni XXII - vd. Zacour 1957, pp. 456-462.

71 Benché non sembri esservi relazione, va segnalata la versione della fondazione di Pavia da parte di un Papirio/Papieno paladino di Carlo Magno, diffusa all'inizio 
Ma la constatazione aiuta a mettere in prospettiva le testimonianze che abbiamo fin qui raccolto, mettendoci in guardia da un anacronismo. Fin dal XIII secolo, in Vincenzo di Beauvais, le notizie relative ad Alcuino e agli altri discepoli di Beda nonché la pagina di Notker sui due monaci irlandesi erano deliberatamente poste in linea di continuità istituzionale con lo Studium parigino; lo stesso non avveniva, invece, per Pavia. La «Ticinensis urbs» è per così dire ospite non invitata in questi bozzetti storici dedicati a Parigi, senza che la notizia dell'invio di uno dei due monaci mercanti di sapienza al monastero di Sant'Agostino desse adito a stabilire un nesso di continuità con un attuale Studium Papiense. ${ }^{72}$ Che questo non avvenisse prima del 1361 è comprensibile (e può anzi essere una conferma del fatto che fra il XIII e il XIV secolo un generale Studium qui non esistesse). ${ }^{73} \mathrm{Ma}$ un accostamento del genere non si trova nemmeno nello Zabarella, che scrive verso il 1400, quando lo Studium visconteo è già florido.

La trasfigurazione della notizia dei due mercanti in leggenda di fondazione dello Studium Papiense non si trova nemmeno - per passare a un genere letterario diverso da quello giuridico - nell'influente Summa bistorialis di Antoninus Florentinus (1389-1459), cioè il frate domenicano Antonino Pierozzi, arcivescovo di Firenze dal 1446 (canonizzato nel 1523). Nella secunda pars della narrazione parenetica della storia dell'umanità dalle origini fino al suo tempo, e precisamente nel contesto delle vicende di Carlo Magno, sant'Antonino inserisce un apposito paragrafo «De studio Parisiensi quomodo sit institutus» e vi ripete per intero - sulla scorta della cronaca di Arles usata da Vincenzo di Beauvais e poi perduta - la pagina di Notker Balbulus. ${ }^{74} \mathrm{Il}$ sincronismo con la fondazione di Parigi e insieme la credibilità del racconto

del Duecento dalla cronaca di Pietro Codagnello e di lì ripresa ancora all'inizio del secolo successivo: vd. Majocchi 2008, pp. 151-161 e 247-248.

72 Ciò, ovviamente, a mia conoscenza. A volte, il racconto veniva abbreviato, e cadeva anche la menzione di Pavia: così nel commento di poco successivo a quello dello Zabarella steso da Giovanni da Imola 1525, c. 126v, Ad Cle. 5.1 .1 v. Inter, n. 5.

73 V'era però, almeno dal XIV secolo, un ben strutturato Studium domenicano (nel quale saranno stati letti anche i commenti alle Clementinae), di cui dà notizia sicura, autobiografica, Galvano Fiamma (1283-1344) nel Chronicon maius (Fiamma 1869, p. 771 ed. Ceruti).

74 Sant'Antonino 1527, Pars II, Tit. XIV, cap. IV, $₫$ XII, c. $125 r-v$. Su di lui, Howard 1995. 
risalente al monaco di San Gallo uscirono sicuramente rafforzati dall'autorità di cui godette la cronaca di sant'Antonino presso i lettori quattrocenteschi, ma ancora non si vede emergere un collegamento fra il racconto di Notker e l'origo dello Studium Papiense.

Il nesso manca a maggior ragione anche nella storia dei Franchi di Robert Gaguin (1433-1501), dove si ricorda (in un contesto analogo al pastiche di Vincenzo di Beauvais) che «Carolus [...] Iohannem vero Papiam misit», ma la sorte del centro di studi pavese non riceve altra attenzione; anzi, la mente del teologo e umanista francese, professore alla Sorbona, corre subito a un'altra sede, ossia a Bologna, la cui maggiore antichità - secondo il falso privilegio di Teodosio - metteva a rischio il primato parigino («Bononiensis schola quae Theodosio imperante initium cepit est aetate superior»): un rischio scongiurato dal Gaguin osservando che tuttavia Bologna era di gran lunga inferiore quanto a docenti. ${ }^{75}$

Qualche parola in più è spesa, nel medesimo giro d'anni, dal Sabellico (ca. 1476-1506), nella sua «rapsodia» di storia universale ( Parisiis divinae humanaeque sapientiae Gymnasium instituit, et in Italia Ticini alterum, ut quibusdam placere video»), ma si tratta ancora di un ragionamento tutto interno alle imprese di Carlo Magno, basato sulle due consuete tradizioni - quella dei due monaci e quella di Alcuino e i tre compagni, di cui Sabellico nota peraltro la difficile compatibilità - senza che la fondazione carolingia sia posta in diretta linea di continuità con quella visconteo-sforzesca: va però segnalato che l'uso del termine Gymnasium lascia almeno trapelare l'idea. ${ }^{76}$

Qualche decennio più tardi, nel 1572, quando il teologo cattolico olandese Jakob Middendorp (1537-1611) scrisse quello che si può con-

75 Gaguin 1504, 1. IV, c. 28v. Su di lui, Collard 1996.

76 Sabellico 1528, 1. IX, c. 198v; su Marc'Antonio Coccio, vd. per tutti Cochrane 1985, pp. 83-86. La sua notizia è utilizzata in collegamento con l'Academia Papiensis nello scarno Catalogus Academiarum Orbis Chirstiani pubblicato ad Ausburg dal Rustenius 1550, s.v. Italia, nr. 6; vd. anche Sacco 1993, p. 300 . È da menzionare anche l'Anglicana Historia di Polidoro Vergilio 1570, 1. V, p. 106 e l. IV, ibid. pp. 91-92: «Achaius [princeps Scotorum] [...] ad Carolum, qui novas conditurus academias, ad se usque $\mathrm{ab}$ extremis orbis partibus eruditos viros advocabat, Clementem et Ioannem homines doctissimos misit, per quos Carolus postea et Lutetiae et Ticini bonas artes cunctos mortales docendos curavit». La prima edizione dell'Historia del poligrafo urbinate è del 1534; cfr. Ruggeri 2000. 
siderare il primo trattato sulle Università su scala europea - seppure in chiave apologetica - la credenza della fondazione dello Studio di Pavia da parte di Carlo Magno era invece ormai diffusa: «Papiensis sive Ticinensis Academia a Carolo Magno non diu post Parisiensem erecta creditur». ${ }^{77}$ (viceversa, nessuna parola era spesa a proposito dei Longobardi). Lo conferma un'opera scritta nello stesso periodo, anche se destinata a pubblicazione postuma (e a grande successo), il De claris legum interpretibus del reggiano Guido Panciroli (1523-1599): «Academia Ticinensis a Carolo Magno circa anno DCCC instituta». ${ }^{78}$

Questo percorso ci conduce dunque a un interrogativo. Per non cadere in una sorta di illusione ottica, vedendo nelle testimonianze di XIII-XV secolo una leggenda d'origine che ancora non aveva preso forma, occorre chiedersi chi e quando abbia instaurato esplicitamente la connessione, che all'inizio del Cinquecento non era stabilita e settant'anni dopo era una credenza diffusa. Un'indagine capillare, specialmente fra gli scrittori più vicini allo Studium Papiense, che possono essere stati indotti, per chauvinismo, a compiere il collegamento, potrebbe chiarirlo al di là d'ogni dubbio: ${ }^{79}$ indagine che dovrebbe tenere conto del ruolo che venne assumendo, anche in sede locale, il richiamo politico alla tradizione carolingia, a discapito di quella longobarda. ${ }^{80}$ Si può tuttavia già avanzare un'ipotesi, pronta a essere smentita da altre

77 Middendorp 1572, pp. 213-214; cfr. Id. 1602, pp. 45-49. La storia delle accademie era inserita in una più generale riflessione pedagogica, svolta in un trattato sull'Officium scholasticum, ispirata al nesso fra sapientia e religio quali fondamenti essenziali della respublica Christiana («sublata eversaque sapientia, inermis et nuda religio facilius opprimatur»); la dimostrazione stava nella resistenza opposta al luteranesimo dalle città della Germania «ubi Academiae probataeque scholae et bonarum artium studia extabant»: Id. 1583, ep. ded., c. 3v. Cfr. Benz 2002, pp. 105-130; sul superamento della storiografia universitaria del Middendorp da parte del Conring, vd. Zedelmaier 2003, pp. 74 ss.

78 Panciroli 1637, p. 495; vd. anche p. 210.

79 Per fare un solo esempio, esso manca nell'orazione tenuta da Baldassare Rasini ( $†$ 1468), che pure ne avrebbe dato spunto perché tocca il tema della nobilitazione dello Studium («quibus radijs haec vera nostra achademia illustrata sit»: ed. Hammer 1940, p. 143.

80 Sulla figura di Carlo Magno nelle tradizioni medievali italiane, fondamentale Fasoli 1974, pp. 891-916, ma senza riferimento a questo caso; da ultimo, in particolare sul ruolo della leggenda di Carlo Magno in rapporto alla Prima Crociata, vd. Gabriele 2011, con altra bibliografia. Per la questione longobarda, vd. ad es. Mantovani 2005, pp. XI-LXV; Artifoni 2007, pp. 297-304; Majocchi 2008, pp. 117-225. 
letture, ma che ha, mi pare, alcuni seri argomenti dalla sua parte.

Nel 1502, nel quadro di un forte afflusso di studenti francesi seguito all'occupazione nel 1499 del ducato di Milano da parte del re di Francia Luigi XII - che appoggiava la forza delle armi alla pretesa legittimazione dinastica - si laureò a Pavia in utroque Iure il borgognone Barthélemy de Chasseneuz (1480-1541). Il Chasseneuz (Cassaneus nella latinizzazione letteraria) si trattenne anzi dopo la laurea per alcuni anni nel ducato di Milano - iscritto nel Collegio dei doctores - al servizio di Charles II d'Amboise de Chaumont, muovendo i primi passi di una notevole carriera che, al rientro in Francia, ad Autun, nel 1506, lo portò fino alla presidenza del Parlamento di Provenza nel 1532. Morì, a quanto pare, avvelenato da un mazzo di fiori. ${ }^{81}$

Il Chasseneuz fu giureconsulto innovatore, estensore nel $1517 \mathrm{di}$ quello che viene considerato il primo commento sistematico al droit coutumier francese (consuetudines ducatus Burgundiae). Nel 1529 diede alle stampe un'opera d'altro tipo, e di fama universale. Fra i molti modi di dare un senso al mondo - oltre che attraverso il diritto - c'è anche quello di ordinarlo gerarchicamente secondo l'onore, le lodi, il decoro che spettano a ciascuna cosa o persona: è questo il punto di vista adottato dal Chasseneuz nel Catalogus gloriae mundi. «Nulla è più utile nella vita - sosteneva l'autore - che conoscere l'ordine e il perimetro della propria condizione»; infatti «sebbene tutti siamo per natura uguali, tuttavia a causa della colpa alcuni sono superiori, altri inferiori [...] così che i superiori possano punire le colpe, e conducano i sudditi alla concordia». ${ }^{82}$ Il Catalogus gloriae mundi, la cui prima edizione è del 1529, rispondeva insomma a un intento moraleggiante e conservatore, proiezione, sul piano intellettuale, di una società fittamente parcellizzata e stratificata per ceti (cui cerca di dare sistemazione filosofico-giuridica), e di un assetto assolutistico del potere. Da giurista, il Chasseneuz sapeva anche che il suo Catalogus avrebbe avuto grande utilità pratica

81 Sulla sua biografia, resta fondamentale Pignot 1970, spec. pp. 14-33 per Pavia e l'esperienza italiana; cfr. Picot 1915, pp. 45-46. Sull'ideologia giuridica e politica, vd. per tutti Randall 2008, pp. 148-168; Goria 2010, pp. 19-21, nega giustamente che sia stato allievo di Claude de Seyssel. Sul valore umanistico della gloria, Varotti 1998.

82 Chasseneuz 1529, I, praef., c. $2 v$ : «nihil utilius in vita comperiet quisquam, quam noscere ordinem et fines sui status»; ivi, c. $3 r$ : «licet omnes natura simus aequales, culpa tamen exigente, alii sunt superiores, alii inferiores [...] ut superiores culpas puniant, et subditos ad concordiam ducant». 
dato che frequenti e assidue strepitano contese sull'onore, le precedenze, la maggiorità, la superiorità, e sul titolo delle dignità e nobiltà, o riguardo all'ordine delle sedute, nei consigli, nelle congregazioni, nei collegi, nei convivi, nel foro giudiziario, e in altri luoghi, persino quando si cammina per le strade. ${ }^{83}$

«Persino quando si cammina per le strade»: la memoria letteraria torna facilmente al fatale incontro che avrebbe cambiato la vita di fra Cristoforo.

Un dilemma che poteva porsi, fra gli infiniti, è chi fra due doctores dovesse precedere ad esempio in un corteo o in una seduta di laurea. $\mathrm{Di}$ questo si occupava, fra l'altro, la decima parte del Catalogus del Chasseneuz ${ }^{84}$ Un criterio per stabilire la posizione poteva fornirlo la disciplina: così fra i letterati erano preferiti i teologi, fra i giuristi i canonisti, secondo una graduatoria che non faceva che protrarre, in cifra minore, le ben note contese fra le arti, già conosciute dall'antichità e rinverdite dall'Umanesimo, a cominciare dal Petrarca ${ }^{85} \mathrm{Ma}$ fra due doctores di una stessa scienza, come stabilire il ranking? La casistica allineata da Chasseneuz era infinita: i docenti precedono quelli che non insegnano; fra i docenti, prevalgono quelli con stipendio maggiore; quelli che hanno scritto libri; il più anziano precede il giovane, ma - onore al merito - se il più giovane è «in scientia excellens» viene preferito al più anziano. In una società che conosceva il valore legale del titolo (la «licentia ubique docendi») si teneva conto anche del valore dell'Università, perciò «doctor creatus in maiori, famosiori et dignori Universitate sit aliis praeferendus». ${ }^{86}$ Quest'ultimo criterio tuttavia riapre il problema, perché impone di stilare una classifica delle Università. Il Chasseneuz non si sottraeva, adden-

$83 I v i$, c. $4 r$ : «materia huius operis est multum commendabilis, necessaria, usitata, et practicata, cum frequens et assidua circumstrepit quaestio de honoribus, praecedentijs, maioritate, superioritate, ac titulo dignitatum aut nobilitatum, aut super ordine sessionum: tam in concilijs, congregationibus, collegijs, convivijs, in foro praetorio, et in alijs locis: etiam per vicos deambulando».

$84 I v i$, c. $4 v$ : «dignitates, praestantias, et praeeminentias scientiarum, tam inter se, quam ad alias res, et ordinem praecedendi inter viros literatos enucleabit». Sulla logica che presiede a questa decima pars, vd. Füssel 2011, pp. 84-85. Sulle questioni d'onore, vd. per tutti Bellabarba 1999, pp. 225-250; per la trattatistica sui duelli, forma estrema di soluzione, vd. Cavina 2003.

85 Vd. per tutti Garin 1947; Avellini 1990.

86 Chasseneuz 1529, X, cc. 19r-22v: è la trigesimasecunda consideratio della decima pars; la cit. ivi, c. $19 r$. 
trandosi in una comparazione che è per noi di grande importanza. Il punto di partenza era fuori discussione: i dottori in Teologia, Diritto canonico e Medicina dell'Università di Parigi erano da preferire a coloro che avessero conseguito altrove la laurea nelle medesime discipline. L'Università di Parigi era infatti «maior, dignior et antiquior» rispetto a tutte le altre. Siccome la storia - sotto forma di origo - è un titolo di grande valore, Chasseneuz s'appellava alla fondazione della scuola parigina a opera di Carlo Magno, avvalendosi della testimonianza di Notker Balbulus (che gli era accessibile proprio tramite gli autori che siamo venuti fin qui conoscendo, lo Zabarella, Antonino Fiorentino e il Gaguin). È a questo punto che la sua penna si volge a Pavia, dove lo scrittore si era laureato appena ventiduenne: ${ }^{87}$

Non voglio tacere che in quello stesso tempo ([in cui fu fondata la Scuola di Parigi] secondo le affermazioni di Gaguin, di Antonino Fiorentino nella sua Storia e anche di Zabarella nei luoghi sopra citati, ebbe invero inizio la Scuola, Università o Ginnasio di Pavia, e perciò può rivaleggiare con l'Università di Parigi quanto alla fondazione avvenuta nello stesso tempo; questo vale per la sincronia. Dico queste cose perché fui alunno e studente pavese studiando sotto il fu professore Giasone del Maino, di venerata memoria, mio precettore eccellentissimo, e ivi ho conseguito il dottorato, con lui, con i professori Franceschino Corti nonché Filippo Decio, e messer Rocco Corti, che furono lì miei precettori esimi per un quinquennio. E perciò non ho voluto tacere l'elogio di quell'Università di Pavia, che mi nutrì, educò, e formò in Diritto canonico e civile. ${ }^{88}$

Queste righe ci fanno entrare direttamente nell'officina del mito, che prende forma, se non sbaglio, sotto i nostri occhi. Come sappiamo,

87 Passò le Alpi nel 1497, dopo avere iniziato gli studi a Dole e a Poitiers, fermandosi dapprima a Torino; con l'occupazione del ducato da parte di Luigi XII si spostò a Pavia. Ancora studente, ventenne, fu nominato magister ordinarius requestarum del luogotenente del re e assessore del capitano di giustizia Robert de Pradines (esperienza cui fa riferimento in Chasseneuz 1529, X, c. $19 v$ e c. $18 v$, ove è attestata l'età in cui conseguì la laurea a Pavia - insieme all'incarico di assessore del capitano di giustizia, che già ne segnalava l'eccellenza - proprio perché è anch'essa un criterio di precedenza). Nel 1506 la peste lo spinse a rimpatriare.

88 Chasseneuz 1529, X, c. 19v: «Nolo praetermittere, quod hoc etiam tempore ex dictis Gaguini, Anto. Florentini in sua hystoria et etiam Zabarellae in locis praeallegatis, schola, universitas seu Gymnasium Papiense habuit saltem initium, ex quo contendere potest cum universitate Parisiensi de institutione eodem tempore facta, et sic 
i due autori quattrocenteschi citati dal Chasseneuz, e il più vicino Gaguin, non avevano sostenuto che Pavia fosse stata fondata da Carlo Magno tramite uno dei monaci irlandesi: essi si interessavano solo di Parigi. Dunque, il Chasseneuz recupera da essi solo la pagina di Notker Balbulus, ma è egli stesso a trarne le conseguenze, nell'ottica del suo Catalogus: quel che vale per Parigi deve valere anche per Pavia, che perciò sono pari almeno per quanto riguarda l'età («ex quo contendere potest cum Universitate Parisiensi de institutione eodem tempore facta, et sic de paritate temporis»).

$\mathrm{Fu}$ il Chasseneuz a fare il passo che mancava negli auctores da lui citati: se l'ipotesi è giusta, abbiamo dunque incontrato - caso non frequente - l' "inventore" di una tradizione. Che a dare forma al mito carolingio di fondazione dello Studium Papiense sia stato un alumnus dello stesso Studium, che in questo modo acquistava un titolo di preminenza sui doctores di altre Università nell'ottica del Catalogus, offre anche un movente soggettivo all'operazione.$^{89} \mathrm{Su}$ un piano più generale, si noterà che il collegamento con il re dei Franchi è compiuto da un borgognone, dopo che il ducato di Milano era entrato nell'orbita della Francia, mentre ancora le grandi potenze europee non avevano cessato di combattere per il possesso dell'Italia, all'indomani della battaglia di Pavia..$^{90}$ Vale la pena di ricordare, simmetricamente, che proprio nel 1529, al

de paritate temporis. Et haec dico eo, quia fui alumnus, et scholaris Papiensis sub quondam recolendae memoriae domino meo Iasone de Mayno praeceptore meo excellentissimo, et ibi doctoratum accepi sub eo, et domino Francisquino de Curte necnon domino Philippo Decio, ac domino Rocho de Curte, qui fuerunt ibidem praeceptores mei eximij per quinquennium. Et ideo nolui tacere laudem illius Universitatis Papiensis, quae me enutrivit, educavit, et aluit in iure Canonico, et civili»; il testo prosegue: «postquam tamen habuerim prima cunabula in Universitatibus Dolana et Pictavensi de quibus infra dicitur». Le citazioni esplicite ai luoghi degli autori citati sono nel passo precedente, riferito a Parigi.

89 La conclusione generale si legge in Chasseneuz 1529, X, c. $21 r$ : «in iure civili magis aestimantur doctores iurium creati in Italia, maxime Papiae, Bononiae, Paduae, quam creati in Gallia, nisi sint creati in Universitate Aurelianensi [cioè di Orléans], ubi maiori rigore examinis examinantur quam alibi». Diverso l'elenco di un analogo trattato di circa un secolo posteriore, steso sempre da un giurista, Jakob Andreas Crusius (Hannover 1636-1680), che fra le italiane inserisce Padova al primo posto, seguita da Bologna, quindi Pavia, Pisa, Siena, Perugia, e menziona la fondazione carolingia solo a proposito di Parigi. Vd. Crusius 1665, pp. 835-836; sulla sua opera, Füssel 2011, p. 85.

90 Sugli studenti stranieri, in particolare borgognoni, vd. Rosso 2012, pp. 402-408. 
ritorno dalla prigionia spagnola seguita al rovescio, Francesco I prese la decisione di istituire i primi «lecteurs royaux», cioè fondava il Collège de France, realizzando il progetto coltivato da Guillaume Budé: un altro sincronismo, in questo caso frutto culturale di quell'avvicinamento francese all'Umanesimo che è un risvolto delle guerre d'Italia. ${ }^{91}$

L'elogio di Pavia contenuto nel Catalogus - come vedremo subito - ebbe immediata eco, diffusa dal successo europeo dell'enciclopedia moraleggiante del Chasseneuz, continuamente ristampata e propagata dal Middendorp, nell'altrettanto celebre rassegna delle più celebri Accademie europee. ${ }^{22}$ Passò con essa anche la datazione - incongrua fissata dal Chasseneuz al 740: «sic ergo ex praedicti constat, quod hae universitates scilicet Parisiensis et Papiensis habuerunt saltem initium tempore Caroli Magni Francorum Regis circa anno Domini 740».93

Naturalmente, non si può escludere che altri prima dell'alumnus Papiensis Chasseneuz avessero compiuto l'accostamento. Tuttavia, varrà la pena di tornare a un testo già citato, quello del giurista Giovan Battista Costa edito nel 1610, dove sosteneva che la «gloria» - il termine è già significativo - dell'Università di Pavia dipendeva dalla sua antichità: anche il Costa, con la capacità diagnostica tipica del giurista che soppesa le prove, leggeva allo stesso modo il testo del Chasseneuz, distinguendo le sue fonti dal suo personale apporto alla instaurazione del mito.

\section{InTERMEZZO CINQUECENTESCO: DALL'EUROPA A PAVIA}

Il Cinquecento pavese è un secolo spezzato in due dalle vicende della guerra franco-spagnola, culminate nel sacco del 1527. La storia politica e la storia culturale tuttavia non coincidono necessariamente; Pavia conoscerà in questo stesso tempo alcuni dei suoi

91 Sulla storia del Collège de France, vd. la Garanderie 2006, pp. 125-142; Tuilier 2006c, pp. 145-163: i corsi iniziarono nel marzo 1530; in particolare sui rapporti con l'Umanesimo, italiano, ma anche spagnolo, vd. Tuilier 2006b, pp. 7-52.

92 Chasseneuz è citato, per l'origine carolingia, ad es. da Alessandro da Rho 1586, Index, s.v. Gymn. Tic. e da Costa 1610, p. 299. Dipendono invece dal Middendorp, Andrés Mendo 1655, p. 5 e Johann Friedrich Heckel (1640-1715 ca.), nelle note apposte alla riedizione dell'Atlante universale del Clüver 1697, 1. III, cap. XXV, p. 244.

93 Chasseneuz 1529, X, c. 19r. Lo segue nella datazione Costa 1610, p. 299. 
maggiori scienziati, dall'Alciato al Cardano. Vero è tuttavia che un'organizzazione «leggera», anzi volatile, com'era uno Studium, aggregazione di docenti e studenti, era in balia delle condizioni circostanti; pativa immediatamente le ristrettezze delle risorse destinate agli scopi bellici e il venir meno delle condizioni di sicurezza che garantivano le peregrinationes.

La crescente incertezza degli anni che precedono la battaglia di Pavia si riflette vivacemente in una commedia incompiuta dell'Ariosto - I Studenti - stesa verosimilmente nel 1518-1525. ${ }^{94}$ Claudio, studente veronese che ha ascoltato a Pavia le lezioni di Messer Lazzaro («che vi leggea la sera l'ordinaria»), si è innamorato della figlia del maestro presso il quale dimorava, che perciò l'ha allontanato da casa (con la falsa imputazione d'un omicidio occorso «in le pratiche del Rettor»). ${ }^{95}$ Trasferitosi a Ferrara, il giovane Claudio riceve da un amico (che a sua volta aveva avuto l'informazione dal padre Bartolo, nome che rivela in modo trasparente l'ambientazione nella Facoltà giuridica) la notizia che proprio nella città estense sta per trasferirsi il suo antico professore Lazzaro, con famiglia e - quel che più conta - figlia al seguito:

[...] egli mi avisò che messer Lazzaro, poi ch'a Pavia levato era 'l salario alli dottor, né più si facea Studio per le guerre che più ogni dì augumentano, avea tramato, pel mezzo di Bartolo, suo padre d'esser condotto qui a leggere e che l'avea ottenuto; et era in ordine con tutta la famiglia per venirsene. ${ }^{96}$

94 Vd. Coluccia 2001, pp. 27; 131 ss.; sarà completata con il titolo La Scolastica dal fratello Gabriele e con il titolo L'imperfetta dal figlio Virginio. Ai vv. 396-398 riferimento alla caduta di Ludovico il Moro avvenuta vent'anni prima.

95 La turbolenza della vita notturna di Pavia nell'ultima età sforzesca («latrociniis et cedibus ea urbs plena erat, quibus scholares et etiam Germani inmixti putabantur») e la sua instabilità politica («fluxa et mutans [...] Papie respublica»), ma anche il prestigio dei suoi docenti e la maggiore semplicità dei modi, rispetto al più formale $\mathrm{e}$ altrettanto valido Studium padovano («minor vestium pompa»), ove erano anche più aspre le contese per il rettorato, sono rievocati proprio all'inizio del 1500 da un professore di Diritto canonico a Ingolstadt, Sixtus Tucher in una notevole epistola a Anton Kress, che s'accingeva a scegliere fra le due sedi, riprodotta e commentata da Walther 2008, pp. 275-276.

96 I Studenti, Atto I, s. 1, vv. 110-115: Ariosto 1974, pp. 627-697, ed. Casella. 
I versi ariosteschi descrivono con precisione quel che aveva indotto Messer Lazzaro a lasciare Pavia e a cercare altrove una condotta: con il crescere delle guerre, il salario non viene più garantito e lo Studio è infine interrotto. Del resto, proprio nel giro d'anni in cui l'Ariosto scriveva la commedia, precisamente nel 1524, s'arrivò a fondere lo scettro d'argento del rettore per coniare il denaro onde pagare i soldati che difendevano la città sotto assedio. ${ }^{97}$ Narrerà Arnoul Le Ferron nelle Res gestae Gallorum che i pavesi sotto assedio costruirono macine di fortuna in tutti i luoghi della città,

persino nell'Accademia del Diritto civile e pontificio. Perciò le urla rozze dei mugnai strepitavano in un luogo nobilissimo dove pochi anni prima erano state ascoltate le voci di Giasone, dei Deci, dei Corti, dei Pichi, e se ne era ammirato l'acume. ${ }^{98}$

Se le guerre avevano svuotato le aule (e attratto incidentalmente sullo Studium l'attenzione degli storici), la nuova sistemazione del ducato sotto il dominio spagnolo portò con sé la ripresa: già nel 1545 si poteva pensare a ricostruire lo scettro rettorale. ${ }^{99}$ Nei decenni che seguono, le politiche culturali della Controriforma individueranno anzi in Pavia un centro in cui investire, accentuando la rifioritura.

97 Le Ferron 1550, 1. VII, c. 96r-v colloca l'evento alla fine dell'anno o all'inizio del 1525: nella sua narrazione, il sacrificio degli scettri rettorali (al plurale: «argenteos baculos») rappresenta il culmine di una requisizione che ha già coinvolto persino gli arredi sacri. Vd. anche Parodi 1753, p. 48, che trae la notizia dal Ballada. Una descrizione di tono diverso della città, che ancora nel 1523 «pare una Roma», è nella lettera, forse edulcorata a uso della destinataria, del segretario di Baldassarre Castiglione edita da Poletti 2009, pp. 327-333.

98 Le Ferron 1550, 1. VII, c. 95r: «trusatiles molas [...] in ipsa academia civilis pontificiique iuris. Ita pinsorum voces inconditae nobilissimo loco strepebant, ubi paucis ante annis Iasonis, Deciorum, Curtiorum, Picorum, cum admiratione acuminis auditae fuerant». Franceschino Corti junior registra i fatti nella dedica di un commento al Codice di Giustiniano: «cum igitur sic fatis impellentibus proh dolor evenerit Ticinense Gymnasium simul cum urbe maximum naufragium fecerit, in quo et captivitatem cum tota familia et maximam iacturam bonorum passus sum [...]»: Corti iun. 1533, praef. Sul Corti, che passò a insegnare a Padova, vd. Piovan 2007, pp. 79-88, che documenta le conseguenze economiche affrontate per il pagamento del riscatto, in cui fu sovvenuto dal fratello Matteo, docente di Medicina teorica.

99 La lettera di Carlo V del 24 novembre 1545 al vicario del vescovo di Pavia è riportata integralmente da Informatio 1611-1617, pp. 14-15; cfr. Parodi 1753, p. 56. 
Di questo parla proprio la scheda che Jakob Middendorp dedicò a Pavia nella già citata rassegna delle celebriores Academiae Orbis Christiani, uscita per la prima volta nel 1572 e che segnò una svolta nella conoscenza comparata delle Università. La scheda si apre appunto con la credenza della fondazione carolingia: «Papiensis sive Ticinensis Academia a Carolo Magno non diu post Parisiensem erecta creditur». Come già sappiamo, Middendorp scrive dopo il Chasseneuz. ${ }^{100}$ Un collage di espressioni adulatorie spigolate dalle epistole dedicatorie premesse a opere di docenti pavesi, come Giasone del Maino e l'allievo Rocco Corti, serve al Middendorp a indicare l'acme del prestigio. Di lì, la storia recente prende il segno della decadenza, determinata dalla guerra franco-spagnola (con la fusione degli scettri rettorali che Middendorp attinge dal Ferron), che ha comportato anche la dispersione della «egregia bibliotheca» del Castello. La curva negativa si chiude, all'altezza del 1572, con una nota - apologetica - di ottimismo: «per munificenza e generosità in parte dei pontefici Romani, in parte di altri principi si è risollevata: infatti Papa Pio IV ha istituito a Pavia un bellissimo Collegio», s'intende il Borromeo. ${ }^{101}$ Suggello della parabola è la paretimologia «Papiam quasi piorum virorum patriam dicas» (che, potremmo dire, realizza l'auspicio dell'Azario che lo Studium contribuisse a riscattare la città «parum pia»)..$^{102}$

Fra le sue fonti pavesi, il Middendorp cita Bernardo Sacco (1497-1579), ${ }^{103}$ dai cui libri De Italicarum rerum varietate et elegantia, editi poco prima, nel 1565, trasse l'etimologia di Papia, una notizia topica sul clima che favorisce l'ingegno e una su san Siro. La citazione del Sacco da parte del teologo olandese ci spinge a rientrare dal-

100 In prospettiva apologetica, Middendorp sottolinea che la scuola pavese era stata da Carlo destinata «ad conservandam propagandamque veram doctrinam Christianae religionis». Ė rivelatore dell'asimmetria della leggenda di fondazione, che per Pavia ci si accontenti si rinviare all'esposizione delle fonti e del racconto svolto a proposito di Parigi: Middendorp 1572, pp. 332-334; del resto le fonti qui citate sono quelle tradizionalmente incentrate sulla scuola parigina.

101 «Sed partim Pontificum romanorum, partim aliorum principum munificentia et liberalitate iterum constitit. Pius enim PP.IV pulcherrimum Papiae collegium instituit».

102 Middendorp 1572, p. 214; per l'Azario, vd. supra.

103 Sacco 1587; traduzione di Domenico Magnino in Sacco 1993. Sull'autore e l'opera, Pasi Testa 1980, pp. 229-250; Magnino 1993, pp. 5-6; Gabba 2000, pp. 17-27; 35-37. 
l'orbita europea, in cui eravamo entrati seguendo il Chasseneuz, per fare ritorno a Pavia e verificare a che punto stesse l'elaborazione del discorso sull'origo.

In effetti, il Cinquecento è, per Pavia, il primo secolo della storiografia cittadina, che si apre con il Papie Sanctuarium di Jacopo Gualla (1505) e si chiude con l'Historia dei vescovi di Pavia e dei «fatti notabili occorsi in tutto l'universo» di Antonio Maria Spelta (1597). Lo Studium non è tuttavia tematizzato direttamente e anche di traverso riceve modesta attenzione. Le prime prove della storiografia pavese seguono disegni precisi, ben chiariti da Emilio Gabba, nei quali l'istruzione pubblica non entra. ${ }^{104} \mathrm{E}$ vero che, a una lettura meno cursoria, il Middendorp avrebbe potuto trovare nel Sacco qualche notizia più specifica, come l'episodio al quale lo storico pavese ricordava d'avere assistito «da ragazzo», quando

Ludovico re dei Francesi e duca di Milano, mosso dalla fama di Giason del Maino, si fermò a Pavia per sentire una lezione di Diritto di un uomo così famoso. Entrato nell'aula, con la mano fece silenzio e impedì che i giovani studenti gli facessero posto. Si sedette dunque, egli che era re, come fosse uno di loro, alla presenza di cento nobilissimi maggiorenti. Ascoltato Giasone lo elogiò, e all'uscita lo invitò, a titolo d'onore, a stargli a fianco a capo coperto, poi lo onorò con il dono di una toga dorata, cioè intessuta di fili d'oro. ${ }^{105}$

L'aneddoto rivela tuttavia nel Sacco un interesse per la dignità sociale che deriva dal dottorato, piuttosto che per il Gymnasium Ticinense e per le sue vicende. ${ }^{106}$ Le origini, su di lui, sembrano eserci-

104 Gabba 2000, pp. 11-32;33-37; sullo Spelta in particolare, Cavagna 1980, pp. 251-263.

105 Sacco 1993, 1. VIII, cap. 7, pp. 228-229 (trad. Magnino); lo scrittore, nato nel 1497 , non potè essere fra gli ascoltatori. Curiosa l'annotazione che segue, apparentemente riferita alla didattica: «si potrà opportunamente apprendere il diritto per filo $\mathrm{e}$ per segno se si ha cura di scrivere: ma di questo parlerò forse più opportunamente in altro luogo».

106 Lo stesso episodio è narrato con verve da Giovio 1553, p. 125, testimone addirittura di uno scambio di battute: «io udii un tratto Lodovico Re di Francia che l'addimandò, per qual cagione ei non havea mai preso moglie; a cui egli rispose: acciò che raccomandandomi tu a Papa Giulio d'un cappello, ei sappia, che ho impedimento alcuno al Cardinalato. Et fu quel giorno medesimo, che vestito d'una roba di broccato d'oro lesse la sua lettione», della quale il Giovio riporta l'argomento. 
tare più fascino del progresso: la versione ormai corrente, che vuole Carlo Magno fondatore, è accolta anche dallo storico pavese. ${ }^{107}$

Di poco maggiore è lo spazio che riserva all'Università Stefano Breventano (1501-1572) nella Istoria della antichità, nobilità, et delle cose notabili della città di Pavia edita nel 1570, in quanto «cosa degna di farne memoria» nel «dilettevole et maraviglioso spettacolo» offerto dalla città. ${ }^{108} \mathrm{Il}$ Breventano tocca tre punti: ${ }^{109}$ si sofferma sull'origine, che fa anch'egli risalire a Carlo Magno sulla scorta del racconto di Notker Balbulus, conosciuto apparentemente attraverso il Gaguin e che Breventano infioretta di dettagli senza riscontro (il monaco Giovanni Scoto fu sepolto in San Pietro in Ciel d'Oro e teneva le lezioni «vicino alla chiesa di S. Maria Perrone», che è invece uno dei luoghi dove si svolgevano le lezioni dello Studium visconteo); descrive poi finalmente testimonianza di prima mano - l'aspetto attuale delle scuole «fatte molto magnifiche dalli Duchi Sforceschi», ossia l'edificio a due cortili costruito da Ludovico il Moro intorno al 1488 in Strada Nuova; ${ }^{110}$ infine, presenta lo svolgimento di una giornata di lezioni, scandita dalle materie che si succedono nelle scuole «de Legisti» e in quelle «de gli Artisti».

La selezione dei temi compiuta dal Breventano è emblematica: da una parte, il lungo affondo rivolto alle mitiche origini; dall'altra, un fotogramma che fissa il presente, l'edificio che era sotto gli occhi di tutti in Strada Nuova e la quotidianità delle lezioni. Manca qualsiasi riflessione sulle vicende dal 1361 in avanti, sui rapporti con la città, sui contenuti dei corsi. Al paragone dà maggior rilievo - propiziato dall'esserne il Bidello - all'Accademia degli Affidati, fondata «pochi anni sono da alquanti nobili cittadini per honore della patria [...] la quale fino al presente con gran fama e riputatione persevera». ${ }^{111}$

In realtà, il Sacco (e il Breventano che da lui in molta parte dipende) scriveva in un contesto fortemente influenzato da una contesa scoppiata alcuni anni prima fra Pavia e Cremona per ragioni di precedenza, di quelle, per intenderci, alle quali il Chasseneuz aveva dedicato il suo

107 Sacco 1993, 1. X, cap. 18, p. 300. La fonte tuttavia è il Sabellico; da notare che la fondazione a Pavia viene attribuita a un desiderio di onorare i Longobardi.

108 Breventano 1570, risp. cc. 10r; $12 v$.

109 Ivi, cc. $12 v-13 r$.

110 Vd. Mantovani 2012.

111 Sugli Affidati, vd. Pissavino 2013 e Repossi 2013. 
Catalogus gloriae mundi. ${ }^{12}$ Le loro opere ricorrevano perciò quasi programmaticamente alla storia, anche la più antica, in funzione di titolo di nobiltà (anche se, nel Sacco, questa finalità era inserita in un disegno ideologico più ampio, imperniato sulla centralità della Roma cristiana). ${ }^{113}$ Casus belli era stata la visita a Milano di Filippo II nel 1548, ospite di numerose cerimonie cui erano invitati i rappresentanti delle varie città; di qui le agitazioni per ottenere il migliore posto nei cortei, nelle sedute, nei discorsi. La contesa fra le due città padane raggiunse l'acme nel 1550, quando a sostenere le rispettive ragioni scesero in campo due scrittori di diversa autorevolezza ed età, ma entrambi di forte tempra: Cremona reclutò il celebre poeta e umanista Marco Gerolamo Vida, vescovo di Alba (1480-1566), autore fra l'altro di un poemetto sul gioco degli scacchi celebre in Europa; ${ }^{114}$ Pavia si affidò a Giulio Salerno (ca. 1524-1554), giovanissimo docente di Istituzioni nello Studium, che meritò poi d'essere chiamato alla cattedra di Diritto civile a Padova, prodromo d'una auspicata carriera preclusa dalla morte. ${ }^{115}$

Anche se alimentata da concreti interessi del presente (il criterio per deciderla cui s'appellavano più volentieri le autorità spagnole era la quota del gettito dovuto al Fisco, sulla base della ripartizione del 1546), la vertenza - le cui sequenze sono state tratteggiate un secolo fa da Ezio Levi, e che si protrasse in varie forme fino al $1785^{116}$ - fu combattuta dagli oratori delle due parti ricercando credenziali soprattutto nel passato.

È a questo proposito che compare il nome di Carlo Magno. Il

112 Per la polemica contro il Vida, principale oratore dei cremonesi, vd. ad. es. Sacco 1993, 1. III, capp. 7-9, pp. 92-101; i riferimenti sono continui: vd. Levi 1905b, pp. 160-165.

113 Gabba 2000, p. 19.

114 Vd., con notizie biografiche, l'edizione del Ludus Scacchiae (Vida 1975) e del De arte poetica (Vida 1982).

115 Levi 1905b, pp. 147-152, con un giudizio non favorevole sulla qualità letteraria dei discorsi ricalcati «troppo servilmente» su quelli del Vida, anche se non disconosce «una grande diligenza nella discussione delle testimonianze arrecate, una non ispregevole erudizione», giustamente notata già dal Capsoni 1785, II, pp. XIII-XIV.

116 Vd. Levi 1904; Id. 1905a; Id. 1905b, anche per lo sfondo socio-economico che premeva sotto alle questioni di forma; importante anche Politi 2002, pp. 122 ss.; Meschini 2001, p. 122, nt. 65, rievoca la polemica seguita alle accuse rivolte dal Vida contro l'attendibilità di Bernardino Corio, citato dai pavesi a sostegno delle proprie pretese. I discorsi del Vida - mandati a stampa a differenza di quelli del Salerno (Vida 1550) - furono addirittura banditi dal governatore Ferrante Gonzaga, con l'obbligo per chiunque le detenesse di consegnarle; pari sorte subì una Responsio Ticinensium: Levi 1905a, pp. 4 22. I discorsi del Salerno, incaricato fin dal 1550, furono stesi nell'estate del 1551. 
Salerno - che possiamo fare qui parlare nella traduzione allestita nel 1798 da Giuseppe de' Necchi Aquila ${ }^{117}$ - riteneva ovviamente che la presenza dello Studium fosse «il più grande giudizio [emanato] da sommi principi sulla dignità» di Pavia. I cremonesi, consapevoli dell'importanza del tema, replicavano con puntiglio, rinfacciando agli avversari innanzitutto che il finanziamento gravava sull'intera fiscalità dello Stato di Milano (al che replicava in prospettiva tutta campanilistica il Salerno: «che importa allo splendore ed alla fama di una Città il rilevare onde traggano le loro mercedi i Professori?» $)^{118}$ e poi ritorcevano la fondazione a «biasimo e contumelia» dei pavesi, in quanto l'Università sarebbe stata fondata in mezzo a loro «ad ammollirne la ferità» essendo «uomini atroci e crudeli». Per rispondere il Salerno poteva usare a nuovi scopi la leggenda d'origine: «Dite, per vostra fede: allorché in Parigi egualmente ed in Pavia Carlo Magno le pubbliche scuole in pari tempo istituiva, rinfacciava egli forse, siccome a noi, anche ai Parigini, la povertà, la barbarie?»

Questi stralci sono sufficienti a dare la misura del raggio ancora limitato entro cui, ancora a metà Cinquecento, il passato dell'Università riusciva a essere tematizzato nella storiografia locale, anzi di come esso rimanesse spesso bloccato nella morsa del campanilismo e della giuridicizzazione. D'altra parte, si ha qui la testimonianza della ricezione in sede locale del mito «inventato» (o almeno portato a compimento) una ventina d'anni prima dal Chasseneuz, compreso il sincronismo con Parigi. Ricezione ovviamente favorita, nel clima della Controriforma, dal fatto che Carlo Magno era l'imperatore chiamato dal papa a scacciare proprio i Longobardi dall'Italia. ${ }^{119}$

117 Vd. il ms. Orazioni di Giulio Salerno Patrizio Pavese dette in Senato in difesa della sua Patria contro le tre invettive di Mons. Vida Cremonese, Tradotte da G. de' Necchi Aquila, l'anno 1798 (BUPv, Ticinesi, 434). La traduzione rimasta inedita era preordinata a un'edizione commentata, il cui piano è riepilogato in una lettera a Siro Comi, da Milano, 11 settembre 1799 (ivi); il testo latino e italiano a fronte sarebbe stato corredato di «note storiche, e letterarie: Gianorini intorno a queste mi prometteva molte notizie circa ai soggetti che furono professori nella nostra Università, e voi mi avreste favorito circa agli altri». Il de' Necchi (nato nel 1755), rimasto sempre estraneo all'Università e con incarichi amministrativi per lo più a Milano e Lodi, morì già nel 1800: preciso ritratto in Volpi 2000, pp. 407-412.

118 Questa e le seguenti citazioni sono tratte dalle Orazioni di Giulio Salerno tradotte 1798, III, pp. 22-26 = Oratio latina III, pp. 144-148.

119 Sacco 1993, spec. 1. X, cap. XVIII, pp. 297-301, è documento importante dell'imbarazzo nel giustificare il ruolo avuto da Pavia come capitale longobarda. 
È ancora un riflesso della contesa la decisione di ristampare l'opera del Sacco presa dal governo cittadino nel 1587, aggiungendovi un encomio dedicato specificamente all'Università. ${ }^{20}$ Autore ne era un giurista e letterato di fama, originario di Liegi, Enrico Farnese (Henri Du Four), docente di Retorica in Università dal 1580 alla morte nel 1613, fra i più prolifici protagonisti della cultura pavese a cavallo dei due secoli, curatore, fra l'altro, dell'edizione rimasta canonica degli Statuta

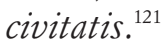

Al di sotto del ridondante fraseggio latino, l'orazione sviluppa un ragionamento di un certo interesse, rivendicando un ruolo politico allo Studium e perciò alla città. Farnese insiste nell'attribuire all'struzione universitaria il ruolo di promozione della concordia sociale e di sostegno al potere politico e al buon governo: «quis non videt reipublicae fundamentum in literarum et virtutum institutione esse positum?». ${ }^{122}$ Se Atene con la sua libertà di discussione aveva suscitato dissidi, il modello pavese è quello di una scienza ispirata dalla rivelazione cristiana, sotto il controllo del vescovo (in quel momento Ippolito de' Rossi, che era stato capace di opporsi alla pretesa di san Carlo Borromeo di sottomettere la sede di Pavia alla potestà metropolitica dell'arcidiocesi di Milano). La funzione che svolge è del resto iscritta nella sua genesi: sono imperatori e papi, con i loro privilegi, i veri costruttori dell'Università, ben più importanti degli architetti che ne hanno fabbricato gli edifici. ${ }^{123}$ La rivendicazione della centralità politica dello Studium pavese, che spicca nel panorama nazionale, e verso cui confluiscono Francia, Germania e Italia, arriva a fare dire al Farnese che la «dignitas» del re Filippo II diventa visibile - s'intende nel suo spettacolo multietnico - nei «cortili delle virtù» dell'Università ticinese come forse in nessun altro luogo al mondo («vix ullo orbis loco gloriosius, quam in his vestris virtutum claustris»).124 Qui «confluiscono tutte le nazioni come ad un emporio delle ottime arti»,

120 Encomium De Singulari et eximia Papiensis Academiae praestantia, in Sacco 1587, pp. 261-272; la ristampa fu inoltre corredata - nella medesima prospettiva di esibire i titoli di merito della città - da un opuscolo dello stesso Sacco sulla dignità della Chiesa pavese e dai privilegi concessi dai pontefici ai vescovi di Pavia.

121 Sull'ideologia e la presenza culturale del Farnese, vd. soprattutto Pissavino 1986, pp. 83-106; censimento delle opere in Cavagna 1981, p. 83.

122 Farnese, in Sacco 1587, p. 261.

123 Ibid.

124 Ivi, p. 265. 
significativo paragone - che riattiva la figura dei monaci mercanti di sapienza - fra mercato e Università. ${ }^{125}$

Il Farnese, nel suo realismo, stila un inventario anche delle risorse materiali che assicurano l'eminenza di Pavia: oltre ai benefici del clima (che con ardita eziologia connette all'appellativo di Ciel d'Oro: «Papiae ita est tenue et salutare, ut caelum aureum a summa eius praestantia sit nominatum») l'oratore passa in rassegna i Collegi allora attivi, Castiglioni, Griffi, Marliani, dedicando un occhio di riguardo al Collegio Borromeo (non un Collegio, ma addirittura un mondo «tanta arte et regio apparatu») e al Ghislieri, nonché al Bossi, che si distingue non per opulenza, ma per i meriti degli studenti: il che consente al Farnese, con accortezza politica, di elogiarne il presidente, che era allora Simone Bossi senatore milanese. ${ }^{126}$

Anche l'ormai classico riferimento a Carlo Magno viene colorato di una nuova tinta: la sua scelta - commenta il Farnese - fu dettata dalla consapevolezza che l'istruzione è necessaria al governo, «magistra per quam reges regnant». ${ }^{127}$ Carlo Magno trasse anzi più prestigio dall'Università, di quanto l'Università dal suo fondatore: anche la maggior longevità dello Studium rispetto al transeunte potere del re franco lo dimostra. Il corollario, per quanto quasi provocatorio, è esplicito: «se il maggior presidio del regno sta nella cittadella della scienza, e se la scienza del governo discende dall'istruzione, quanto valore deve attribuire Filippo, re dei re, a Pavia, vera madre della virtù e della cultura?». ${ }^{128}$ Insomma, Pavia rivendica la propria centralità nello Stato di Milano, e anzi con una proiezione addirittura sull'intero sistema imperiale spagnolo: centralità non di forza militare o economica, ma ideologica, centro di irradiazione di una cultura funzionale agli assetti di governo, concepita quasi come potere sullo stesso piano di quello sovrano: «est Papia quasi quoddam tabernaculum omnium scientiarum, Regum atque Imperatorum socia, Principum magistra, Senatorum procreatrix». ${ }^{129}$

125 Ivi, pp. 265-266: «ad quod tamquam ad optimarum artium mercaturam confluunt nationes omnes et provinciae».

126 Ivi, pp. 266-267.

127 Ivi, p. 269.

128 Ivi, p. 268: «si in doctrinae arce summa regni praesidia sunt posita, atque ars omnis reipublicae administrandae alumna est rei literariae, quanti ab ipso regum rege Philippo fieri Papia, cuius vera germanaque suboles virtus est et res literaria?».

129 Ivi, p. 270. 
L'ambizione politica di quest'encomio del Farnese ${ }^{130}$ si misura anche a paragone di altri discorsi epidittici pressoché coevi, come ad esempio l'orazione di laurea tenuta nel 1553 da Aldigeri Cornazzano, già ricordata per il legame che stabilisce fra la tradizione longobarda e quella carolingia. ${ }^{131}$

Secolo della storiografia pavese, il Cinquecento si chiude mostrando la difficoltà a rapportarsi con l'effettivo passato dello Studium in termini anche solo elementari. Ne dà prova il già citato Alessandro da Rho, lettore peraltro ben radicato nell'Università, in cui si era laureato (nel $1569)^{132}$ e dove tenne la lectura Iuris civilis ordinaria vespertina, salvo un intervallo a Pisa, dove Ferdinando de' Medici gli elargì la precedenza fra i lettori. ${ }^{133}$ Quando nel Tractatus de analogis, univocis et aequivocis (edito nel 1587), in un obiter dictum, elencò i primi professori di Diritto dello Studium, il da Rho incorse in una serie d'abbagli cronologici. Secondo il suo calcolo, Cristoforo Castiglioni, Uberto Lampugnani, Baldo e Raffaele Fulgosio avevano insegnato «ab hinc annos 290, sicque fuerunt primi qui legerunt post erectionem huius Gymnasij factam a Comite virtutum

130 Varie orazioni accademiche furono raccolte dal Farnese nel De simulacro reipublicae, trattato di teoria politica (sulla cui ideologia vd. Pissavino 1986, pp. 83-106) organizzato per panegirici, ciascuno dedicato a una istituzione (es. principe, città, senato, magistrati), a una virtù (es. clemenza, verità, religione, pietà), a un'attività (es. agricoltura, commercio), infine alla famiglia e ai suoi componenti (incluse le singole arti e professioni). Ogni panegirico svolge il tema da angolature ricorrenti, incominciando dalle insegne d'una famiglia illustre e appoggiandosi via via a motti, simboli, allegorie e riti, che rendono memorabile ciascuno dei concetti esaminati. La studiata architettura è un recipiente in cui trovano posto anche alcune prolusioni accademiche, collegate tematicamente a singoli panegirici. Esse insistono piuttosto convenzionalmente sui contenuti delle singole discipline, con intonazione parenetica piuttosto che politica: si veda ad es. De ignorantiae detestatione. Oratio Ticini, pro felicibus studiorum auspicijs, babita, in Farnese 1594, 1. II, pp. 101-107; collegata significativamente al tema dell'oboedientia) oppure De dignitate Eloquentiae, et Praestantia oratio, pro felicibus studiorum auspicijs Ticini habita (ivi, 1. III, pp. 118-127, collegata al tema dell'honor), nella quale peraltro non manca qualche eco della funzione politica dell'istruzione.

131 Cornazzano 1643, pp. [1-2]; fu Accademico degli Affidati: vd. Regali 1999, p. 233. L'edizione dell'oratio un secolo dopo, da parte di Angelo Cornazzano, è segno di un'idea di continuità intergenerazionale.

132 Arese Lucini 2008, p. 209: senatore dal 1574 al 1590. I dettagli sulla cerimonia di dottorato, su promotori e praeceptores in Alessandro da Rho 1587, p. 389, nr. 354-355; Id. 1595, cons. 22, c. $213 r$.

133 Memorie e Documenti, I, p. 83. 
Vicario Imperialis, et ab Urbano Pontifice approbatam, anno 1291». Nessun papa Urbano era sul soglio nel 1291, ma il computo di 290 anni trascorsi (che porta al 1581) conferma che Alessandro da Rho era convinto che proprio quella fosse la data di istituzione (che del resto sarà ripetuta da autori posteriori). ${ }^{134}$

L'infortunio non è però il sintomo di un disinteresse. ${ }^{135}$ In una postilla all'indice della medesima opera, sotto la voce Gymnasium Ticinense (che rimanda al luogo poco sopra riportato), Alessandro da Rho cita la fondazione di Carlo Magno (attribuita anche qui a un impossibile «anno a partu Virginis $740 »)^{136}$ e aggiunge:

quo tempore in eo explicabantur leges consuetudines ac constitutiones Longobardorum, ut retulit mihi Illu(strissimus) et Clariss(imus) Iurisconsultus affinis meus D. Co(mes) Alphonso Beccariae constare ex antiquissimo libro manuscripto penes ipsum existente, monacorum Cassianensium Divi Salvatoris Papiae, tractante de institutis, et Regalibus legum Longobardorum. ${ }^{137}$

Il riferimento, come già sappiamo, è al manoscritto delle Honorantie civitatis Papie, che qui riemergono dopo un silenzio di quasi due secoli dopo lo stralcio riprodotto da Filippo Maria Visconti nel decreto di riapertura dello Studium nel $1412 .{ }^{138}$ Questa postilla, pur gra-

134 Non meno impreciso il suo allievo, Melchiorre Alciato 1600, pp. 29-30, nr. 60-66, che accoglie egli pure la fondazione carolingia; il trattato De praecedentia è per vari aspetti istruttivo sull'uso giuridico della storia.

135 Alessandro da Rho e l'udinese Nicola Graziano, professore di Civile ordinario dal 1566 al 1588, intrapresero una controversia contro il Collegio dei giudici di Pavia, che li escludeva in quanto non cittadini, con conseguenze sulla loro partecipazione alle attività accademiche. Gli atti della causa rivelano una messe di conoscenze sulla prassi e la normativa, secondo quanto segnala Zorzoli 1986, p. 182, nt. 96. Per una sua orazione, convenzionale, De praestantia civitatis Gymnasiique Ticinensis, vd. Alessandro da Rho 1592.

136 Alessandro da Rho 1586, Index, s.v. Gymn. Tic., afferma di trarre la notizia dal De consuetudinibus del Cassaneus, ma in realtà la ricava dal Catalogus gloriae mundi.

137 Trad.: «In quel tempo in esso [nel Gymnasium] si spiegavano le leggi, consuetudini e costituzioni dei Longobardi come risulta - secondo quanto mi ha riferito il mio congiunto, l'illustre e chiarissimo giurista Conte Alfonso Beccaria - da un antichissimo libro manoscritto che è presso di lui, appartenente ai monaci Cassinesi di San Salvatore a Pavia».

138 Dubbi a mio parere ingiustificati in Brühl - Violante 1983, p. 8, che affermano che il Raudense non entra minimamente nel contenuto; in realtà «institutis, et Regalibus legum longobardorum» è proprio la citazione dell' incipit delle Honorantie, con la lezione legum per regum. Ivi, p. 7, per una possibile precedente citazione nel Gualla. 
vata d'errori, annoda la fondazione carolingia escogitata dal Chasseneuz alla presenza di una scuola di Diritto longobardo (che l'interpolazione delle Honorantie lasciava intendere). Alessandro da Rho anticipava così, quasi prodigiosamente, uno schema che, come vedremo, dovrà attendere il XIX secolo e la riscoperta dell'Expositio ad Librum Papiense per essere messo a punto. D'altra parte, il riferimento a un manoscritto - quello dei monaci di San Salvatore posseduto da Alfonso Beccaria, lo stesso che aveva esortato il Farnese a pronunciare l'encomio dell'Università - è il presagio di una nuova generazione di studiosi, che inizierà a cercare negli archivi il passato dello Studium, come Gerolamo Bossi ${ }^{139}$ e Pietro Francesco Scarabelli: ${ }^{140}$ un passato inteso però non (solo) come origo, bensì come progressio, come tempo reale dopo (e non prima) il 1361.

Queste interessanti «prove di storia»-adumbratae historiae per usare parole dello Scarabelli - sono segnali del nuovo atteggiamento verso le fonti che matura nel corso del Seicento, di cui sono emblema i padri benedettini di Saint Maur: occorre perciò ora fare un passo avanti e considerare la sorte dei miti di fondazione esposti alla «lucerna» della critica ${ }^{141}$.

139 Notizie biografiche sul Bossi (1588-1646), in Castronovo 1971, pp. 305-307; preciso profilo culturale in Repossi 1995, pp. 712-713. Fra le sue carte, fondamentale per le notizie d'archivio raccolte sono le schede riunite nel ms. Studio (BUPv, Ticinesi, 181), che rivelano anche un disegno complessivo di storia dell'Università. I suoi scavi archivistici erano ammirati (e utilizzati) già da Capsoni 1785, II, p. XV; basti dire che l'elenco dei rettori stilato dal Bossi (ms. Studio, cc. 8-15) fu recepito dal Robolini 1836, V, 2 pp. 272-283 (fino al 1447) ed è quello che si legge, ricontrollato dal Prelini, in Memorie e Documenti, I, pp. 5-15. Per compilarlo, il Bossi s'avvalse per il XVI secolo specialmente dell'Informatio pro Bilio Episcopo 1611-1617, sulla quale vd. nt. seguente.

${ }^{140}$ Lo Scarabelli, lettore di Medicina Teorica e preposto dal Senato di Milano all'archivio universitario (notizie su di lui nella scheda di Gianorini, in BUPv, Ticinesi, 527), aveva progettato una Historia Gymnasii ticinensis, che rimase però allo stadio di progetto («adumbrata historia» l'autodefinisce), conservato in ASMI, Studi p.a., 158 (sono grato a Alessandra Ferraresi per la segnalazione). Il progetto era destinato a ricondurre la prassi dello Studium in conformità alle norme, e fallì. Rinvio ad altra sede per una trattazione complessiva, segnalando qui che il Seicento si caratterizza anche per alcune importanti raccolte di norme a fini giudiziari, mandate a stampa, segno di una nuova sensibilità archivistica: vd. in particolare Informatio pro Bilio Episcopo 1611-1617 e Decreta et Rescripta 1618, in connessione con le controversie fra podestà e vescovo in merito alla competenza sull'Università, e Responsa, et Instructiones 1612, in tema di immunità.

141 Per tutti, Raimondi 1989 e Frova 2012, pp. 14-16. 


\section{I «GENTILISSIMI ARTIGLI» DI ANTONIO GATTI}

L'idea vulgata che il Seicento non sia stato altro che declino è da rivedere, anche per il Gymnasium Ticinense. Non c'è dubbio, tuttavia, che in quel secolo l'Università sia stata all'attenzione del Senato di Milano più per ragioni di ordine pubblico che di indirizzo culturale. La facilità con cui si portavano le armi, le contese di precedenza e il punto d'onore, le proteste per il pagamento degli stipendi e le richieste di immunità monopolizzano i carteggi e di conseguenza assurgono alla ribalta della documentazione storica, rubando la scena ai segni della affaticata, ma pur costante vitalità del Gymnasium Ticinense. Non è da sottovalutare che per un ventennio, in fine secolo, ne sia stato sopraintendente un fine intellettuale come Carlo Maria Maggi, segretario del Senato di Milano e perno della vita letteraria milanese. ${ }^{142}$

Sullo scorcio del secolo, mentre muoveva i suoi primi passi proprio nella cerchia del Maggi, il giurista Antonio Gatti si volse a illustrare «antiquissimi huius Gymnasii gloria, sua vetustate non leviter fatiscens», segnando una tappa importante nella riflessione sul passato dell'Ateneo, sia pure entro i limiti che vedremo.

Quella del Gatti è una figura complessa, che conviene brevemente tratteggiare, per quel che la sua personalità può rivelare dell'opera. ${ }^{143}$ Nato nel 1671 a Garbagna, presso Tortona (dove morirà nel 1752), ${ }^{144} \mathrm{da}$ famiglia distinta e con una tradizione nell'avvocatura, assunse il ministero sacerdotale e compì anche gli studi giuridici, addottorandosi a Pavia nel 1696. Da subito sospeso in un difficile equilibrio intellettuale e sociale fra avvocatura, erudizione e ambizione, entrò nella cerchia letteraria che si riuniva a Milano intorno al Maggi e nell'Accademia dei Faticosi e annodò un fitto rapporto personale ed epistolare con il pressoché coetaneo Muratori (allora dottore della Biblioteca Ambrosiana). ${ }^{145}$ Proprio la

142 Cipollini 1900, pp. 305-318; cfr. Bufacchi 2006, p. 330.

143 Sul Gatti, De Bernardi 1936, p. 7; fondamentale Pecorella 1964, pp. 53-65; approfondimenti in Zorzoli 2000, pp. 359-390; vd. anche Mantovani 2004, pp. 248-257; sintesi in Dezza 2008, pp. 791-814.

144 Cfr. Parodi, Syllabus: PAVIA - ARChIVIO DI STATO ASPV, Università, Studium Ticinense, cart. 17, vol. I, s.v.

145 Rispetto all'ed. Càmpori, di cui si poteva avvalere Pecorella 1964, p. 64, nt. 37 , si dispone ora di vari volumi dell'Edizione Nazionale (non ancora di quello relativo al Gatti). Ferraglio 2010, pp. 151-168, dà notizia della dispersione delle lettere muratoriane al Gatti appartenute a Vincenzo Poggi e già edite dal Càmpori e calcola in un centinaio le lettere del tortonese presso la Bibl. Estense. 
parola «ambizione» ricorre subito nel carteggio con il Vignolese. ${ }^{146}$

L'abate Gatti cercò anche la legittimazione universitaria, adattandosi a tenere gratuitamente a Pavia dal 1701, ormai trentenne, la lettura De actionibus (cioè sul processo) basata sulle Istituzioni di Giustiniano. ${ }^{147}$ Fu quindi chiamato, nel 1703, alla lettura straordinaria delle Istituzioni, per approdare, nel 1707, alla cattedra di Pandette - vacante da oltre 40 anni, perché non essenziale - lungo una linea che lo qualifica dedito agli aspetti storici del diritto piuttosto che a quelli applicativi: una linea che era congeniale alla sua ambizione di fare parte della «repubblica letteraria d'Italia» chiamata a raccolta dal Muratori. ${ }^{148}$

Un rapporto, quello con la repubblica letteraria, in cui Gatti si fece presto conoscere per i suoi «gentilissimi artigli» e che lo vide incappare in ripetuti incidenti. ${ }^{149}$ Già nel 1703 s'era distinto per una pomposa contesa con un altro docente pavese per una questione di preceden$\mathrm{za}^{150}$ e s'era sentito rispondere a tono, come commentava il pur amico

146 Muratori a Gatti, 7 ottobre 1700 (Càmpori 1901, p. 469, nr. 420): «Cominciano a deporsi i pensieri filosofici, e si prendono quei delle Corti, dopo che l'esempio mio ha fatto grande invidia alla vostra ambizione». Il tortonese ambiva a una promozione a Vicario Generale, per la quale aveva chiesto il sostegno del Muratori.

147 Non mi è noto se il Gatti sia stato agevolato dal Maggi per la chiamata, avvenuta però dopo la morte del sopraintendente. Compare fra i membri dell'Accademia istituita in casa Borromeo - fra i quali il governatore Henriquez de Cabrera, Alessandro Litta e il Muratori - che si radunarono il 23 aprile 1699 per commemorare in prose e versi italiani e latini la morte del Maggi (Cipollini 1900, p. 318).

148 All'orientamento umanistico dell'insegnamento il Gatti si dichiarò fedele, pur ammettendo la tiepida accoglienza riservata dagli studenti ai campioni della giurisprudenza culta, con delusione proporzionale alla sua ambizione di affermazione culturale: Gatti 1704, dedicatio, p. [a3r] = 1722, p. [2].

149 L'espressione è di Francesco Arisi (l'autore della Cremona literata) al Muratori (27 luglio 1697), in Marcocchi 1975, p. 59, nr. 64; la missiva segue quella del 24 luglio 1697 all'Arisi (ma destinata ad essere mostrata anche al Gatti), nella quale il Muratori annuncia di «aver preso la spada contro de' Pavesi» i quali «si stimano ingiuriati ch'abbia nominato quella città con l'autorità di S. Ennodio lor vescovo angustia oppidi Ticinensis» (ivi, p. 58, nr. 63). È appunto a proposito del De antiquo Iure Metropolitae Mediolanensis in Episcopum Ticinensem (che confuta la pretesa originaria libertas della Chiesa pavese dal vescovo di Milano: «hanc unam crebro cantilenam Ticinenses Scriptores canunt») che il Gatti si riprometteva di «mortificare» il Muratori sfoderando quelli che l'Arisi chiama appunto i suoi «gentilissimi artigli».

150 Con il professore di Prassi criminale, Juan Angelo de Apezteguias: vd. la Diatriba pubblicata dal Gatti 1703, su cui Pecorella 1964, p. 54; Zorzoli 2000, pp. 359-366. 
Francesco Arisi al Muratori: «Il nostro dott. Gatti con una diatriba stampata in Pavia in occasione di precedenza con un altro lettore, ha egli attaccato i sonagli alla gatta havendosi tirato addosso la risposta assai pungente». ${ }^{151}$

Ben più serio l'incidente che accadde nel 1709 , per il coinvolgimento del Gatti (finora sfuggito ai suoi biografi, proprio perché già al tempo si cercò di negarlo) in una rovente polemica, fra fede ed erudizione, scoppiata in seguito all'edizione, nel 1707, dei presunti Acta SS. Martyrum Crescii et Sociorum da parte del faentino padre Giacomo Laderchi (uno dei continuatori degli Annales del Baronio). Quando il clero fiorentino chiese alla Sacra Congregazione di Propaganda di poter celebrare la festa dei santi Cresci e compagni - venerati in un oratorio restaurato in Mugello dal granduca Cosimo III - il padre Gerardo Capassi, fiorentino anch'egli, mise in guardia dalla probabile falsità degli Atti e trasse dalla sua parte eruditi del calibro di Leandro Porzia, Giusto Fontanini (che ne parlò come di una «frottola commentata») e persino Benedetto Bacchini. Dopo schermaglie, nel 1709 uscì a stampa un opuscolo preciso nelle critiche all'edizione degli Acta, ma anche colmo di sarcasmo fin dal titolo, Nugae Laderchianae. ${ }^{152}$ Il rumore fu enorme, non solo per la rabbia che scatenò nell'offeso Laderchi: ${ }^{153}$ Cosimo III, punto nel vivo, comandò addirittura che l'opuscolo fosse pubblicamente bruciato (e non mancò chi ne raccolse e spacciò le ceneri quasi miracolose). ${ }^{154} \mathrm{Il}$ Capassi, ritenuto l'autore delle Nugae (che pur non recavano il suo nome), dovette ritirarsi a Roma. ${ }^{155}$

Il punto è che l'opuscolo figurava edito a Genova «accurante $\mathrm{M}$. Antonio Gatto I.C.» e, da quel che emerge da circospette lettere di

151 Arisi a Muratori, Cremona, 10 agosto 1703: Marcocchi 1975, p. 148, nr. 186. Altra volta il Muratori lo redarguiva per l'eccessiva «libertà di vivere, e di parlare»: Modena, 9 dicembre 1700, ed. Càmpori 1901, p. 486, nr. 435.

152 Gatti 1709a.

153 Il Fontanini (26 luglio 1709) scrive: «Il p. Laderchi marchia per Roma con un servitore armato, minacciando vendetta, se escono altre Centurie delle Nugae Laderchianae qui generalmente aggradite. Il Gatti però scrive da Genova, di volerci dare in breve la seconda Centuria» (ed. Clarorum Venetorum ad Ant. Magliabechium Epistulae, 1745, p. 292, nt. 1.

154 Esprime il proprio dispiacere per la pena «obbrobriosa» Muratori a Orsi, Modena, 14 settembre 1709: Cottignoli 1984, p. 441, nr. 560.

155 La vicenda è sintetizzata ad es. da Fabbroni 1781, pp. 235-242, cui pare non fosse noto il ruolo del Gatti. Sul Capassi, vd. anche Dal Pino 1975, pp. 387-391. 
Giuseppe Gioseffo Orsi (cui l'opuscolo era stato a sua insaputa dedicato), di Anton Francesco Marmi, del Muratori e del Fontanini - cioè di una parte eminente della «letteraria lega» cui il Gatti aspirava d'appartenere - egli non era completamente estraneo alla stampa e s'era fatto dunque partecipe di un'operazione avventata. ${ }^{156}$ Come commentò l'Orsi: «l'autore delle Nugae [...] s'allontana egli e s'allontanano i suoi parziali dalle regole di convenienza verso gli amici onorati»; ${ }^{157}$ Gatti, se non l'autore, era fra questi «parziali».

Gli amici si adoperarono faticosamente per trovare al Gatti una via d'uscita ${ }^{158}$ e alla fine fu spinto «a dimostrare con giuramento la sua innocenza». ${ }^{159} \mathrm{Ma}$ anche di questa difesa - accompagnata da un "Manifesto" a stampa - il Muratori non fu soddisfatto. ${ }^{160}$

Sta di fatto che sotto il nome del Gatti le Nugae Laderchianae finirono addirittura nell'Index Librorum Probibitorum. ${ }^{161}$

Non bastò tuttavia questo episodio ad allontanare del tutto il

156 Come sembra ricavarsi dalla lettera citata sopra in nt. 153, il Fontanini aveva saputo dal Gatti che egli era effettivamente curatore della prima Centuria e s'apprestava a farne uscire una seconda. Quanto al contenuto dello scritto, vd. Orsi a Muratori, Villanova, 24 ottobre 1709 (Cottignoli 1984, p. 446, nr. 565): «Le Nugae non son certamente di Garofalo. Ognun le crede, ed io pur le credo, dello stesso Capasso». Le attribuisce al Capassi (con interventi di Filippo Buonarroti) il dizionario delle opere pseudoepigrafe del Melzi 1848, p. 441. Questo gioco delle parti non è certo isolato nella società letteraria del tempo: per un altro caso in cui si dubitò del ruolo di Gatti, vd. Muratori a Marmi, Modena, 25 marzo 1707 (Viola 1999, p. 238, nr. 23).

157 Orsi a Muratori, Villanova, 23 settembre 1709: Cottignoli 1984, p. 443, nr. 562.

158 Le fasi dell' «accomodamento» sono disperse nei rivoli di molti epistolari. Vd. ad es. la lettera di Orsi a Muratori, Villanova, 18 luglio 1709 (Cottignoli 1984, p. 434, nr. 549) e di Marmi a Muratori, Firenze, 3 agosto 1709 (Viola 1999, p. 255, nr. 39) a proposito della «centuria del Gatti, o pseudo-Gatti».

159 Inghirami 1843, p. 377. Il Gatti produsse una «giurata testimonianza» e «ha renduto pubblico colle stampe questo attestato»: ne dà notizia Orsi al conte Vincenzo Ranuzzi, 29 settembre 1709: Cottignoli 1984, p. 445, nr. 564. Vd. anche l'esortazione rivolta da Orsi a Muratori, Bologna, 11 novembre 1709, a elogiare pubblicamente «la dichiarazione fatta dal Gatti, e supporla cagionata prima dal motivo di non apparir autore d'un'opera ignominiosa, poi da quello di non apparir verso di me mal creato col dedicarmela senza mia saputa»: Cottignoli 1984, p. 447, nr. 567.

160 Muratori a Marmi, Modena, 6 dicembre 1709: Viola 1999, p. 264, nr. 47. Il Muratori (parlando delle Nugae allo stesso interlocutore) s'era già detto «stomacato» dalla «lettura di quella satira non ingegnosa, ma furiosa».

161 Index librorum probibitorum 1852, p. 195. Sulla condanna, cenno in Stella 2008, pp. 81-82, ma senza menzione del Gatti. 
Muratori dal Gatti. ${ }^{162}$ La corrispondenza muratoriana rivela tracce del loro rapporto più in là di quel 1710 in cui vengono meno loro scambi epistolari. ${ }^{163}$ In attesa che sia pubblicato il volume dell'Edizione Nazionale dell'Epistolario muratoriano dedicato al Gatti (comprensivo anche delle sue missive), si può già segnalare, ad esempio, che in una lettera del 1716 indirizzata al già citato Francesco Arisi, comune amico cremonese, il Muratori ne parla ancora come del «nostro Gatti» e gli fa pervenire (almeno indirettamente) chiarimenti eruditi. ${ }^{164}$

Proprio il 1710 sembra segnare tuttavia un turning point nella vita dell'abate tortonese, meno attivo sul fronte culturale e più su quello dell'avvocatura. Da quell'anno chiede più volte di essere esonerato dalla cattedra di Pandette e dal 1712, pur conservando nominalmente l'incarico, viene sostituito da Ferdinando Innocenzo Civalerio, un nobile milanese con interessi letterari, attivo anche come ripetitore presso il Collegio Borromeo. ${ }^{165}$ La sua attività sarà da qui in poi dispiegata, con altalenante successo, in controversie politico-giuridiche, soprattutto nell'interesse della Repubblica di Genova, spesso condotte a colpi di indagini storico-diplomatiche. ${ }^{166} \mathrm{La}$ coincidenza temporale di questa svolta nella vita del Gatti con l'incidente delle Nugae Laderchianae date alle fiamme e infine messe all'Indice è difficile da calcolare, ma anche da sottovalutare.

Il breve profilo del Gatti aiuta a situare le Gymnasii Ticinensis

162 Irritazione sembra gli abbia procurato anche l'anonima orazione De recte instituenda iuris Academia, che sospettava del Gatti (1709b) che vi si (auto)elogiava: cfr. Soli Muratori 1773, p. 26 e lettera di Muratori a Orsi, Spezzano, 26 agosto 1709: Cottignoli 1984, p. 438, nr. 555. Lo scritto del Gatti, senza dichiararlo, era una rielaborazione della Epistula di Duareno sul metodo di insegnamento del Diritto (come ho segnalato in Mantovani 2004, pp. 249-251): questa disinvoltura assume maggior rilievo alla luce del gioco delle parti in cui abbiamo visto invischiarsi il Gatti.

163 Così Ferraglio 2010, p. 156; pongono l'interruzione nel 1707 Pecorella 1964 p. 64, nt. 37; Dezza 2008, p. 798.

164 Muratori ad Arisi, Modena, 5 novembre 1716: Marcocchi 1975, p. 190, nr. 264; la richiesta verte sulle origini di Casalmaggiore. Per altri riferimenti al Gatti si vedano ad es. Arisi a Muratori, Cremona, 5 gennaio 1713 (ivi, p. 176, nr. 232); Muratori ad Arisi, Modena, 9 novembre 1713 (ivi, p. 178, nr. 237).

165 Nel 1723 salirà alla cattedra di Pandette Giacomo Parodi: Memorie e Documenti, I, p. 94. Nel De Praerogativis, et praestantia Lecturae Pandectarum, Parodi 1753, s.n.p. [3], non menziona l'interim di Civalerio.

166 Dezza 2008, pp. 809-812. 
Historia, et Vindiciae A Saeculo V ad finem XV, opera apparsa nel 1704. La genesi è rivelatrice (tanto più alla luce dell'incidente che sarebbe capitato nel 1709): pungolo fu la lettura, da parte del Gatti fresco di laurea, di un opuscolo del giurista melfitano Carlo Antonio De Luca - destinato a una certa risonanza $-{ }^{167}$ che fra tante Università più o meno celebri non citava quella di Pavia: «exarsit statim in animo desiderium vindicandi ab oblivione», dal che il titolo Vindiciae. ${ }^{168}$ Vale la pena di segnalare che nel 1695, proprio mentre il Gatti concludeva i suoi studi legali, erano state ritrovate in San Pietro in Ciel d'Oro reliquie subito attribuite a sant'Agostino. Alla diligente indagine pubblicata dal Ghisoni, seguì un accanito dibattito, che attrasse a Pavia nel 1698 persino il padre maurino Bernard de Montfauçon, allievo del Mabillon, e in cui presero la parola anche Giusto Fontanini e il Muratori: è la temperie in cui matura la decisione del Gatti di dedicarsi alle antichità pavesi. ${ }^{169}$

Sul piano del metodo, il Gatti non si avvalse di documenti di archivio, se non in minima parte: pur essendosi sforzato di «monumenta Universitatis undique perquirere», lamentava che gli archivi pubblici pavesi fossero vuoti in seguito alla guerra franco-asburgica e al sacco oppure che i documenti fossero custoditi troppo gelosamente da privati. ${ }^{170}$ Sono giustificazioni che - se si tiene conto delle acquisizioni che più di mezzo secolo prima era stato in grado di compiere Gerolamo Bossi ${ }^{171}$ e se si pensa alla messe che avrebbe raccolto qualche decennio più tardi il suo successore sulla cattedra di Pandette, Giacomo Parodi ${ }^{172}$ - rivelano i limiti dell'impresa.

Le Vindiciae, in realtà, furono scritte lontano da Pavia, durante un soggiorno di meno di quattro mesi a Roma, in un anno compreso fra il

167 Si tratta del De praestantia laureae doctoralis iuristarum: De Luca 1689. Secondo Rovito 1990, pp. 333-334, quest'opera manifesta l'adesione ideologica del De Luca «alla res publica dei togati» e «rappresentò la più completa ed esplicita teorizzazione di un indirizzo che consentì ai giuristi napoletani di contendere vittoriosamente all'antica aristocrazia di spada l'egemonia politica e costituzionale».

168 Gatti 1704, praefatio, p. [a7v] = 1722, p. [3].

169 Il dibattito erudito suscitato dal ritrovamento delle ossa di sant'Agostino è ricostruito brillantemente da Stone 2002, spec. pp. 48-89. Il Gatti 1704, pp. 64-69= 1722 , pp. 20-22, in un capitolo dedicato al monastero come sede della scuola pubblica carolingia, si tiene prudentemente alla larga dalla controversia.

170 Gatti 1704, praefatio, p. [a8r] = 1722, p. [3].

171 Vd. supra, nt. 139.

172 Vd. infra, $\$$ successivo. 
1701 e il $1704,{ }^{173}$ sulla scorta di opere storiche e letterarie cui Gatti ebbe accesso grazie a uomini di cultura come il già ricordato Fontanini (1666-1736) e dell'allievo di questi Domenico Passionei (1682-1761), destinato a diventare cardinale e prefetto della Biblioteca Vaticana e che già aveva gettato le basi della sua celebre biblioteca privata. ${ }^{174}$

Dei diciassette capitoli in cui l'opera si articola, ${ }^{175} \mathrm{i}$ primi tre sono dedicati non all'Università, ma alla città, nobilitata attraverso una raccolta di testimonianze che spaziano dalle origini ai fasti di capitale longobarda: ${ }^{176}$ prologo che fu scintilla per una diatriba con il padre somasco Giovanni Paolo Mazzucchelli (1672-1714). ${ }^{177}$

Dal capitolo 4, il Gatti s'inoltra nella vera e propria questione universitaria, formulando uno dei capisaldi della sua ricostruzione, ossia che già sotto i Goti all'inizio del VI secolo, al tempo di Boezio e di Ennodio, a Pavia fiorirono scuole che, per natura e uditorio (non solo ecclesiastico) e spettro disciplinare («in quibus non solum de Philosophia, et Grammatica, sed de Divina pagina et de Jure Civili agi videtur») avevano già le caratteristiche di Università pubblica; scuole che il Gatti ipotizza addirittura fossero state istituite con privilegio regio di Teoderico. ${ }^{178}$

Il modus procedendi del Gatti è tutto in questo esordio: attribuire alle proto-scuole pavesi, fin dall'ultima romanità, le caratteristiche organizzative e disciplinari proprie dello Studium visconteo. Questi due elementi sono poi inseriti in un filo diacronico: dal VI al XIV secolo, anche nelle fasi di maggiore difficoltà politica, le scuole pavesi non cessarono

173 Gatti afferma che vi si recò dopo che aveva assunto la cattedra di Azioni, nel 1701; il 1704 è l'anno di pubblicazione dell'opera, già diffusa in una sinossi a stampa presentata al papa Clemente XI (che era salito al soglio nel 1700).

174 Di questi contatti dà conto Gatti 1704 , praefatio, p. $[1 r-2 v]=1722$, pp. [34]. Per l'ambiente, anche con riferimento all'accoglienza riservata al Gatti, vd. Caracciolo 1968, p. 33.

175 Importante lettura in Gabba 2000, pp. 37-42.

176 Gatti 1704 , pp. 1-26 = 1722, pp. 1-9. A questa parte soprattutto fa riferimento, nel sottotitolo dell'opera, «et plura de eiusdem Urbis nobilitate».

177 Il Mazzuchelli gli rimproverava di attribuire a Pavia - invece che a Milano l'appellativo di «Roma secunda» che si leggeva su un'epigrafe presso la Porta del Ponte: «Dic prope, qui transis, qui Portae limina tangis I Roma secunda vale, Mundi caput imperiale | Tu bello Thebas, tu sensu vincis Atenas». La lapide, collocata intorno al 1370, era copia di una lapide di fine XII secolo in Porta San Vito (entrambe ora ai Musei Civici di Pavia): vd. Majocchi 2008, pp. 137-139.

178 Gatti 1704, p. $37=$ 1722, p. 12. Sulla cultura tardoantica a Pavia, vd. Gasti 2012. 
mai. Pubblicità, ampiezza disciplinare, continuità sono dunque i tre cardini delle Vindiciae. L'impresa ulteriore del Gatti è raccogliere le testimonianze più disparate che suffraghino l'assunto. Questa impostazione era destinata a imporsi come modello di discussione (al di là ovviamente delle infinite varianti interpretative): l'opera di Arrigo Solmi, La persistenza della Scuola di Pavia nel Medioevo fino alla fondazione dello Studio Generale, che costituisce per così dire il manifesto scientifico delle celebrazioni per gli Undecima Saecularia del 1925, ne è la riproposizione più evidente, e non l'unica. ${ }^{179}$

Una disamina analitica, qui fuori luogo, metterebbe in luce i meriti del Gatti anche nella raccolta delle testimonianze, da allora rimaste all'ordine del giorno (anche se via via arricchite). Basti segnalare la valorizzazione della rimembranza di Alcuino d'avere assistito adolescente (forse intorno al 767) in Papia regali civitate, tappa d'alcuni giorni durante un viaggio verso Roma, a una disputa fra l'ebreo Lullo e un magister Pietro (Pisano), lo stesso che poi acquistò fama come istruttore di Carlo Magno. ${ }^{180}$ Importante l'enfasi posta su Lanfranco di Canterbury come indicatore del livello mantenuto dagli studi nella patria pavese, dove s'era formato al principio dell'XI secolo. Addirittura - secondo Gatti - Lanfranco «dopo avere insegnato le scienze a Pavia, si recò a Parigi, ove riportò e illustrò, secondo maestro pavese, le lettere che vi erano state portate sotto Carlo Magno da Pietro Pisano maestro pavese». ${ }^{181}$ Insomma, Pavia non era debitrice di Parigi

179 Vd. Solmi 1925, pp. 17-42 e specialmente poi Bassi Costa 1951, pp. 5-47; sui Saecularia Undecima del 1925, vd. infra. Il quadro di riferimento critico sulla questione resta il dittico composto dai due studi di Gualazzini 1974, pp. 35-73 e Pagnin 1974, pp. 75-106, in prospettive che accentuano rispettivamente la discontinuità e la continuità.

180 Ep. 172: ed. MGH Epp. 4 (1895), p. 285 (l'epistola è del 799); Gatti 1704, p. $39=1722$, p. 13, legge, con altri mss., Iulius invece che Lullus. Sulla cultura pavese, nel quadro dell'organizzazione scolastica del Regnum italicum in età carolingia, vd. Gavinelli 2012, nonché Cau - Casagrande Mazzoli 1987, pp. 177-217.

181 Gatti 1704, p. 91 = 1722, p. 28: «postquam Papiae scientias docuerat, ivit Parisios, eoque literas sub Karolo Magno a Petro Pisano Papiensi magistro delatas, alter Papiensis magister revocavit, illustravitque». Un brano dove non sfuggirà l'accostamento suggestivo degli aggettivi "pisano" e "pavese". In questo modello, Gatti non si sofferma su quale sia stata la sede dell'insegnamento che, secondo Eginardo (Vita Caroli c. 25: MGH, SS rer. Germ. 25 [1911], p. 30), il diacono Pietro Pisano impartì a Carlo Magno. Sulla formazione di Lanfranco e sulle scuole dell'Italia settentrionale che frequentò, vd. ora per tutti Gibson 1989, pp. 3-17; D’Onofrio 1993; Collins 2007, pp. 20-22; Álvarez de las Asturias 2008, pp. 65-71. 
più di quanto lo fosse Parigi di Pavia: Gatti portava a perfezione il semplice sincronismo del Chasseneuz, la cui mancata citazione, in un'opera tanto dotta, non può che attirare l'attenzione. Su un piano più generale, il Gatti sembra anticipare, sotto questo profilo, la discussione a distanza che avrebbe diviso il Muratori (e il Denina) dal Tiraboschi quanto al ruolo da assegnare, nel Medioevo italiano, alla cultura autoctona oppure agli apporti stranieri. ${ }^{182}$

Il parallelismo fra Pavia e Parigi era metodico, nella sua trattazione: perciò poteva sfruttare il trattato De Parisiensi Universitate scritto una quarantina d'anni prima da César-Egasse Du Boulay (ca. 16101678), ${ }^{183}$ ricavandone fra l'altro il capitolo XII del concilium Parisiense dell'829, nel quale i vescovi esortano a Ludovico il Pio e Lotario a costituire scuole pubbliche almeno in «tribus congruentissimis imperii [...] locis». ${ }^{184}$ In questi tre luoghi - chiosa Gatti - tutti riconoscono Parigi, Pavia e Bologna (anzi, se le prime due sedi sono certe per tutti, alcuni sostituiscono a Bologna Padova oppure Osnabrück, in Bassa Sassonia). E poiché i vescovi chiedono di imitare «morem paternum», cioè l'esempio di Carlo Magno, si avrebbe conferma che la scuola di Pavia era stata fondata per sua iniziativa. ${ }^{185}$

In questo punto, il Gatti sembra fare suo il modo in cui l'Azario - la cui cronaca allora inedita vide in manoscritto alla Biblioteca Ambrosiana - spiegava la nascita dello Studium nel $1361 .{ }^{186}$ Come sosteneva l'Azario a proposito dei Visconti, così, secondo il Gatti, Carlo

182 Vd. infra, $\$$ successivo.

183 Du Boulay 1665, I, pp. 96-97; vd. anche pp. 156-161. È noto il caustico giudizio sul Du Boulay, ma non sul suo libro, espresso da Rashdall 1936, I, p. 269.

184 MGH Conc. 2,2 (1908), p. 675 (è il cap. 79 dell'intero concilium, e fa parte di quei «capitula quae - dicono i vescovi agli imperatori - a vestra pietate adimpleri flagitamus»): «ut morem paternum sequentes saltim in tribus congruentissimis imperii vestri locis scolae publicae ex vestra auctoritate fiant, ut labor patris vestri et vester per incuriam, quod absit, labefactando non depereat, quoniam ex hoc facto et magna utilitas et honor sanctae Dei ecclesiae et vobis magnum mercedis emolumentum et memoria sempiterna adcrescet».

185 Gatti 1704 , pp. $71-75=1722$, pp. 22-25.

186 Gatti 1704, p. 127 = 1722, p. 39: «ut testatur Synchronus Petrus Azarius Novariensis in Chronicis MS. Bibliothecae Ambrosianae dono datis ab erudito Lazaro Augustino Cotta». Sul codice ambrosiano D. 269 inf., vd. Cognasso, in Azario 1927, pp. XXV-XXVII: tramite al Muratori fu il Beretti (su cui infra). Il Tiraboschi (1775, V, p. 59), che leggeva l'edizione muratoriana, contesta che nell'Azario si faccia «spesso menzione 
Magno scelse Pavia perché era città regia e per le favorevoli condizioni del luogo; se nel 1361 fu istituito uno Studium generale, nel IX secolo furono istituite Scholae publicae et universales; nel ruolo di Carlo IV il Gatti fa subentrare ovviamente Carlo Magno e compare persino un'anticipazione di Bonifacio IX nella figura di papa Leone III, quasi a suggellare la partecipazione di entrambe le autorità universali. Per finire, se nel 1361 si legittimava il nuovo Studium riannodandolo - come accenna Azario - a precedenti (longobardi o carolingi), il Gatti risale fino ai Goti per trovare un precedente a Carlo Magno: ogni origine, insomma, ha bisogno di un preludio ancora più arretrato che la legittimi.

Tuttavia, si vede bene il progresso segnato dal Gatti - grazie a un fecondo contatto con la trattatistica secentesca - rispetto al modo in cui fra il XIII e il XVI secolo era sorto il mito della fondazione carolingia dell'Università di Pavia, imperniato, in definitiva sul solo racconto dei due monaci mercanti di sapienza tramandato (anonimamente) dai tempi di Vincenzo di Beauvais. Naturalmente, anche il Gatti riserva il posto d'onore a questo racconto, che tuttavia discute - è significativo attingendo direttamente alla fonte, cioè a Notker Balbulus, il cui Cbronicon nel frattempo era stato edito dal canonista olandese Hendrik de Hondt (Henricus Canisius) nelle sue Antiquae Lectiones del 1601. ${ }^{187}$ Il trattamento è per un altro verso rivelatore: la macchina argomentativa del Gatti supera di slancio le difficoltà opposte dal testo. Il nome del monaco irlandese inviato a Pavia in alcuni manoscritti dei Gesta Karoli Magni è caduto, in altri è «Albinus», ${ }^{188}$ secondo altri autori è «Ioannes»? Gatti non si perde d'animo e conclude che doveva chiamarsi... Giovanni Albino. ${ }^{189}$ Soluzione conciliativa adotta anche per le

dell'affollato numero di scolari, che prima dell'anno 1362 a quella Università concorreva»; ma il Comi 1783, pp. 159-161 e il Robolini 1836, V, 2, p. 27, nt. 1, individueranno il passo visto dal Gatti, pur dandone - il Robolini - una diversa lettura. Il Tiraboschi prosegue a proposito dell'Azario: «la maniera con cui egli ne raconta la fondazione, ci mostra ch'egli era bensì persuaso che ne' tempi antichi fosse stato in Pavia uno studio generale, ma che già da gran tempo esso era interamente cessato»: una lettura, questa, su cui si deve consentire.

187 Canisius 1601, I, pp. 358-428; ivi, p. 361, accoglie il nome «Albinus» per il monaco inviato a Pavia.

188 Si vd. l'apparato critico in MGH SS rer. Germ. n.s. 12 (1959), p. 3, r. 17. L'editore Häfele sceglie di indicare la lacuna: «Alterum vero nomine... in Italiam direxit».

189 Gatti 1704, p. $49=1722$, p. 15 . Accettarle entrambe ha il vantaggio di ribadire, nell'andirivieni del monaco, l'intreccio fra le scuole di Lutetia e di Ticinum. 
varie ipotesi sull'anno della venuta a Pavia (780 o 792), che risolve con un duplice viaggio del monaco. ${ }^{190}$

Superando tutti gli ostacoli - ad esempio il ritardo con cui a Pavia era stato studiato il diritto (perché «praticamente perduto» prima del XII secolo) ${ }^{191}$ oppure il silenzio serbato da Federico Barbarossa su Pavia nella constitutio Habita ${ }^{192}$ - Gatti conclude il percorso: l'Università di Pavia non fu istituita, bensì fu restaurata da Carlo IV nel 1361 ( «Scholas Papienses a Karolo IV non institutas, sed restauratas, ex ipso Caesareo diplomate probatur»). ${ }^{193}$

Prova regina è l'espressione contenuta nel diploma del 13 aprile 1361: i pavesi chiedono all'imperatore la grazia «de instaurando generali Studio», e «instaurare» non è «instituere». ${ }^{194}$ Appiglio lessicale infinitesimale - tanto più che nello stesso diploma si dice poi «errigatur» - ma al quale, vedremo, la discussione tornò molte volte ad aggrapparsi. ${ }^{195}$

L'opera del Gatti di cui abbiamo indicato metodo e principali passaggi - una vera e propria "rivendica", una memoria giudiziale più che un trattato storico - incontrò subito, come vedremo, la critica del Muratori (e di un altro corrispondente del Vignolese, anch'egli professore a Pavia, il somasco Giovanni Gaspare Beretti, che lasciò i suoi dubbi su un esemplare postillato delle Vindiciae ${ }^{196}$ reiterata, alla fine del Settecento, dal Tiraboschi.

190 I principali autori a sostegno delle due opinioni citati da Gatti (1704, p. 53 = 1722, p. 17) sono rispettivamente Mabillon e Polidoro Vergilio.

191 Il Gatti 1704, p. $92=1722$, p. 28, accetta la spiegazione secondo cui il Digesto era stato ritrovato dai pisani ad Amalfi durante una spedizione compiuta in aiuto di Lotario II il Sassone (1125-1137), il quale l'aveva donato ai propri alleati e con uno o due editti aveva poi imposto l'uso del Digesto giustinianeo nei tribunali e nelle scuole (sulla diffusione ancora nel XVIII secolo di questa spiegazione elaborata dal Sigonio, importante Marrara 1984, pp. 227-330). Ciò gli consente di giustificare l'assenza di tracce di insegnamento giuridico a Pavia prima del XII secolo.

192 Gatti 1704, p. $108=1722$, p. 33. Sui tre atti imperiali che dal 1164 riconoscono a Pavia autonomia giurisdizionale e politico-amministrativa, vd. Dezza 1992, pp. 413-414 e Majocchi 2012, pp. 97-103

193 Gatti 1704 , p. $127=1722$, p. 39.

194 Gatti 1704, pp. $127-134=1722$, pp. 39-41.

195 Il Tiraboschi 1775, V, p. 59, annota ironicamente: «Il Gatti sembra trionfare sopra una parola di questo diploma»; l'argomento del Gatti è tuttavia ribadito da Nova 1859, pp. $145-146$.

196 BUPv, Ticinesi, 12. Il volume pervenne a Siro Comi nel 1794, in un passaggio di testimone emblematico del piccolo mondo erudito in cui si tramanda l'attenzione 
Tuttavia, come s'accennava, le Vindiciae hanno costituito un modello interpretativo che è sopravvissuto alle critiche di dettaglio ed è stato via via riempito di contenuti. Alle sparse notizie d'una attività culturale raccolte dall'abate tortonese altre ne sono state aggiunte; soprattutto, come vedremo, la riscoperta della scuola di Diritto longobardo attiva nell'XI secolo, insieme al Capitolare di Lotario, ha dato nuova linfa alla sua tesi.

Lo stesso impianto della più recente Storia dello Studium Papiense potrebbe definirsi tributario dell'impostazione del Gatti, visto che risale all'alto Medioevo: la differenza di fondo - che questa premessa storiografica vorrebbe contribuire a chiarire - è la consapevolezza che non si tratta di cercare un'origo, ma più semplicemente di definire le condizioni culturali del Medioevo pavese e che occorre ragionare per differentiam, prima ancora che per analogia rispetto alla vita dello Studium visconteo. ${ }^{197}$

Un altro merito bisogna riconoscere al Gatti: benché già nel secolo precedente - con i più volte citati Bossi e Scarabelli - e negli stessi suoi anni con il Beretti fosse maturata l'idea di tematizzare l'Università di Pavia come oggetto storico, i precedenti erano rimasti allo stadio di progetti, incompiuti o comunque inediti. L'opera del Gatti diede per prima l'esempio che l'Università rappresenta per Pavia un tema capace di congiungere di nuovo - dopo i fasti lontani di capitale - la storia della città alla storia nazionale ed europea. La ristampa che l'opera del Gatti ottenne nel Thesaurus Antiquitatum et Historiarum Italiae del Graevius, nel 1722, ne aumentò l'efficacia.

Prima di concludere, si può tornare ad annodare l'opera alla biografia. Gatti lamentò di non avere ricevuto buona accoglienza dai pave-

all'Ateneo cittadino. Sul Beretti (1660-1736), vd. Bianchi 1964; Petrucci 1967, pp. 59-61; Tomea 1993, pp. 222-223; Stone 2002, p. 104. Da segnalare, sul piano della storiografia universitaria, l'inedita e incompiuta Scriptorum Academiae Ticinensis Collectanea. Binis dissertationibus, altera de bis Scholis, altera de hac Universitate, praemissis. Insubrum sacri Senatui. Eadem bumillime nuncupat D. Joh. Gasp. Beretta Benedictino-cassin. M. Regius Metaphysicus (BUPv, Ticinesi, 114): si tratta di un tentativo di storia letteraria relativa ai docenti pavesi, che prefigura il Syllabus (anch'esso inedito) del Parodi. Vd. anche Ticinesi, 304, per una più vasta storia letteraria, Pavia erudita.

197 Della Storia dell'Università di Pavia, nata in occasione delle celebrazioni per i 650 anni (per cui vd. anche Mantovani - Valeriani 2011) è stato pubblicato un primo volu$\mathrm{me}$, in due tomi dedicati rispettivamente alle Origini e fondazione dello Studium generale (Almum Studium Papiense, 1,I) e all'Età spagnola (Almum Studium Papiense, 1,II); il secondo volume - in uscita - sarà dedicato ai due secoli che vanno dall'età austriaca ai primi del Novecento. 
si. In coda a un resoconto elogiativo delle Gymnasii Ticinensis Historia et Vindiciae apparso nel Giornale dei Letterati del 1710 - anonimo, ma che non si fatica a credere di sua mano - il Gatti rivelava il disegno di dare alla luce un'opera De antiquitate Urbis Ticinensis, ormai ultima$\mathrm{ta}^{198}$. Tuttavia, disgustato per non avere ottenuto che se ne pagasse la stampa con i denari pubblici e non riuscendo a essere dispensato

dal grave incomodo di alloggiare certi cavalli nelle stanze, dove egli abita, benché ognuno sapesse, quant'egli, benché straniero, più d'ogni altro de' medesimi cittadini aveva operato per gloria di quella città, spinto da afflizione, e da dispetto diede al fuoco questo suo parto, e poi raccolte le ceneri, vi scrisse sopra: Papiensium gloria cineribus restituta. ${ }^{199}$

Non può non colpire l'analogia fra questo virtuale rogo che il Gatti inflisse alla propria opera su Pavia e quello effettivamente subito l'anno prima dalle Nugae Laderchianae sulla piazza di Firenze. L'anonimo bene informato - cioè il Gatti stesso - faceva leva sul «tragico fine di questo libro» De antiquitate Urbis Ticinensis per augurarsi che non dovesse soffrire lo stesso destino un'altra opera del Gatti, che avrebbe dovuto contenere «le Vite degli Uomini illustri» dell'Università. ${ }^{200}$ In effetti, di questa ulteriore storia universitaria del Gatti non ne è rimasta traccia, come di altre historiae solo adumbratae, sfumata probabilmente prima di cominciare.

\section{Muratori E TiRABOSCHI: LA STORIA NON È NELL'ORIGINE}

«Ora dunque tempo è di liberar la verità da tante favole»:201 con questa dichiarazione d'intenti, intrisa d'impazienza e insieme di consape-

198 [Gatti] 1710, pp. 225-252, con ampio riassunto delle Vindiciae. I sei capitoli De antiquitate Urbis di cui viene fornito il prospetto coincidono in realtà con i primi tre delle Vindiciae, con un riferimento aggiuntivo ai privilegi dello Studium. Può essere che il Gatti considerasse i primi tre capitoli editi come una sintesi di materiale più ampio.

199 [Gatti] 1710, p. 252. Prende alla lettera questo racconto Stone 2002, p. 58; più accortamente la crede una "millanteria" Capsoni 1785, II, p. XX, nt. 54: il parallelo con il rogo delle Nugae lo conferma.

200 A quest'opera fa riferimento anche in Gatti 1704, praefatio, p. b3r $r=1722$, p. [2], dove la paragona per impianto al trattato dedicato da Antonio Riccobono all'Università di Padova.

201 Muratori 1751, III, diss. XLIV, p. 2. 
volezza nel proprio magistero, Lodovico Antonio Muratori (1672-1750) aprì un nuovo capitolo nella storiografia sull'Università in Italia. ${ }^{202}$ Qui se ne devono osservare le conseguenze per Pavia, che in questa revisione era direttamente coinvolta.

Vent'anni dopo le Vindiciae del Gatti, nel 1725, il Muratori pubblicò per la prima volta il Capitolare di Lotario del maggio 825, traendolo dal «Codice delle Leggi» dell'Archivio del Capitolo di Modena: ${ }^{203}$ un testo che oggi molti considerano il certificato di nascita dell'Università, ma che per Muratori era invece la pietra tombale sulla leggenda di fondazione carolingia.

Il Gatti (definito dal Muratori «doctissimus», ma senza attestazioni d'amicizia) era direttamente chiamato in causa nelle note apposte all'edizione del Capitolare, quasi che il Muratori volesse sottrargli subito l'illusione che il nuovo documento potesse confermare il castello delle sue ipotesi.

Com'era possibile credere - notava il Muratori - che il monaco irlandese mandato da Carlo Magno avesse impiantato qui un'Università, visto che qualche decennio più tardi il nipote Lotario apriva il suo Capitolare sostenendo che la «cultura è ovunque radicalmente estinta»? ${ }^{204} \mathrm{Ma}$ soprattutto: anche ammesso (e il Muratori era incline a prestare fede alla notizia di Notker Balbulus) che il monaco irlandese (come dopo di lui Dungalo) avesse tenuto effettivamente una scuola «apud Ticinensem urbem», una tal scuola "monocattedra" non avrebbe in alcun modo potuto, per struttura organizzativa e per materie, essere paragonata a una Università, nel senso che il termine assunse soltanto dopo Bologna. Sono dunque due le direttrici critiche, una culturale e una organizzativa, che il Muratori sviluppò poi nelle due Dissertazioni sulle Antiquitates Rerum Italicarum Medi Aevii. ${ }^{205}$

202 Vd. Frova 2012, pp. 14-16.

203 Muratori 1725, I,2, pp. 151-153, in appendice alle Leges Langobardicae. La datazione posta dal Muratori all' 823 , fu stabilita all' 825 da Georg Pertz e dimostrata dal Boretius 1864, pp. 156-157, sulla base della analitica ricostruzione degli spostamenti di Lotario. Sul ms. Modena, Archivio Capitolare, O.I.2, vd. ora l'ottimo commentario all'edizione in facsimile: Leges Salicae 2008.

204 Cito secondo l'ed. MGH Capit. 1 (1883), pp. 325-326: «De doctrina vero, quae ob nimiam incuriam atque ignaviam quorundam praepositorum cunctis in locis est funditus extincta».

205 Edite dapprima in latino nel 1740, furono volte in italiano, in abregé, e pubblicate postume: Muratori 1751, II, diss. XLIII, pp. 588-632; III, diss. XLIV, pp. 1-47. 
Nella dissertazione XLIV, proprio il libro del Gatti fu preso ad esempio di quelle «favole» da cui Muratori voleva «liberare la verità», che si riduceva, a suo avviso, a questo:

non mancò certamente Carlo Magno di promuovere lo studio delle Lettere tanto nella Gallia che nella Germania, ed anche in Pavia. Molto più fece, e l'abbiam già veduto, Lottario I Augusto in Italia, con avere stabilita scuola in varie città. Scuole eziandio v'erano ne' Palazzi Episcopali e ne' Monisterj; e queste si può credere, che non mancassero in Italia. Pure che furono mai sì fatte scuole? Certamente né pur ombra si vide allora di Università, quali oggidì abbiamo. Un solo maestro si contava in cadauna di quelle poche città, e questi anche non insegnava che l'Arti più basse. In Pavia il solo Dungalo, in Ivrea il solo vescovo insegnavano pubblicamente; e nell'altre città si praticò lo stesso. Adesso fin le castella hanno maestri di non men vaglia che quelli. ${ }^{206}$

La critica del Muratori fu ripresa - con toni più fastidiosi, non foss'altro perché veniva per secondo - da Girolamo Tiraboschi (17311794). ${ }^{207}$ Rispetto al Muratori, due sole, ma significative, divergenze. Innanzitutto, il gesuita attacca frontalmente il racconto «favoloso e romanzesco» di Notker Balbulus (le soluzioni che il Gatti proponeva per sanarne le incongruenze equivalevano a «immaginare, o a dir meglio, sognare personaggi e fatti, che non hanno alcun fondamento»). Quest'attacco serve in realtà a rovesciare l'interpretazione del Muratori circa il basso stato culturale dell'Italia, che per il Muratori sarebbe appunto provato dall'invio del monaco irlandese, e poi di Dungalo, in difetto di maestri locali. Per il Tiraboschi, al contrario, Carlo Magno «non mandò straniero alcuno in Italia a tenervi scuola», anzi fu lui stesso istruito da un italiano, cioè Pietro Pisano, e anzi «molti Italiani inviati furono in Francia a farvi risorgere gli studij» (in questo, a dire il vero, si trovava perfettamente in sintonia con il Gatti). Le linee del Muratori e del Tiraboschi - che l'ideologia tende a separare - tornano a unirsi sulla questione di metodo: le scuole che in età carolingia v'erano in Italia, e anche a Pavia in età carolingia non potevano in nessun caso prefigurare le Università intese come «un corpo di professori, che di tutte, o almeno delle principali scienze tengano scuola» muniti del privilegio di autorità sovrana. ${ }^{208}$

206 Muratori 1751, III, diss. XLIV, p. 2.

207 Sul Tiraboschi, per tutti Di Pietro Lombardi 1996; Mari 1999.

208 Tiraboschi 1773, 1. III, capo I, spec. pp. 124-146. Nella seconda edizione, 
Il Tiraboschi rimprovera anche altro al Gatti, ossia che dopo avere impiegato la maggior parte del suo libro a parlare dell'Università di Pavia «quando essa ancora non esisteva, appena giunto al tempo in cui ella fu veramente fondata, ne interrompe il racconto, e ci lascia quasi interamente digiuni delle vicende e delle glorie di quelle celebri scuole». Il Tiraboschi, com'è noto, era interessato alla storia dei centrì di istruzione, come quei quadri in cui si svolgeva l'ampia storia delle scienze in Italia che il gesuita veniva tracciando dall'antichità a tutto il Seicento. Sentiva perciò l'urgenza non solo di lasciare alle spalle la querelle sulle origini, ma anche di passare a una nuova dimensione, quella dedicata ai concreti svolgimenti delle Università, la dimensione cha abbiamo chiamato progressio. Per Pavia mancavano tuttavia le basi, cioè le carte d'archivio: né le aveva procurate - a dire del Tiraboschi Giacomo Parodi, che raccolse sì «grande serie di documenti», ma che «per lo più versano intorno ad alcune leggi pubblicate pel regolamento della Università», riguardo a questioni minute «e soprattutto a un certo onorario de' Capponi, che ad ogni tratto si nomina», senza dare un'idea dello stato culturale e didattico. Con questi limiti, il Tiraboschi tentò una seppur rapidissima storia dell'Università pavese dal 1361, esponendone i «cominciamenti» sotto Galeazzo II, il «fiore» in cui era sotto gli Sforza, quindi la caduta e ripresa nel Cinquecento, in un bozzetto talmente rapido che non supera quello schizzato duecento anni prima dal Middendorp..$^{209}$

Forse non sapeva, il Tiraboschi, che proprio Giacomo Parodi $(1693-1773)$ aveva in gran parte scritto una tale storia letteraria, che non aveva però avuto i mezzi per divulgare: una storia basata su documenti d'archivio, gli Acta - quelli che il Parodi per cinque anni, fra il 1747-48 e il 1753 , aveva pazientemente cercato e integralmente trascritto - dai quali aveva tratto l'elenco dei rettori e dei docenti, per poi sviluppare una «Litteraria historia singulorum Lectorum», composta di schede bio-bibliografiche. ${ }^{210}$ Di questo lavoro fu mandato alle stampe nel 1753 solo il nudo regesto dei documenti, il famoso Elenchus visto dal

aggiunse una nota per elogiare l'«elegante Operetta» del Villa, che aveva accolto la sua opinione, e per segnalare che un «valoroso Avversario», il Comi, era tornato alle posizione del Gatti, senza argomenti davvero nuovi; opinioni che, dunque, il Tiraboschi (1787, III, p. 169, nt. a), riteneva d'avere «già ribattute» nella prima edizione.

209 Tiraboschi 1775, V, pp. 58-63; Id. 1777, VII, 1, pp. 95-96. 
Tiraboschi, con il corredo di scarne liste nominative (Syllabus) di lettori e rettori (e segretari del Senato). Il Syllabus Lectorum, inteso invece come collezione di dettagliate schede bio-bibliografiche dedicate ai professori pavesi, rimase manoscritto, mentre una complessiva storia «ipsius Studii» restò un progetto. ${ }^{211}$

\section{Villa, CAPSONI E COMi: PROVE DI STORIA NELl'EtÀ DELle RIFORME}

Il rapporto con il passato non s'esprime necessariamente per iscritto. Quale rievocazione della storia dell'Università è più eloquente della collezione epigrafica allestita dagli architetti al servizio degli Asburgo, che apposero sulla parete orientale del palazzo universitario le effigi dei grandi docenti (e di qualche studente)? Fu probabilmente Leopoldo Pollack verso il 1790 a distribuire nei due cortili, secondo un canone per la verità estetico piuttosto che cronologico, i monumenti e le lastre tombali prelevate dalle chiese pavesi, dalle quali un'ordinanza del 1786 aveva prescritto si traslassero le sepolture per deporle nei cimiteri extraurbani. ${ }^{212}$

I bassorilievi e i frammenti epigrafici, proprio per la plasticità di talune delle rappresentazioni, fanno rivivere gli antichi maestri e i loro discepoli. Si tratta quasi di una riduzione della storia dello Studium (anzi dell'Archigymnasium, come allora era chiamato) alle sue figure più rappresentative, una continuazione per altra via dell'Historia litteraria di cui il Gatti aveva fatto un (metaforico) falò e che il Parodi aveva lasciato incompiuta (come altri prima e dopo di lui).213

${ }^{210}$ L'utimo eventuale obiettivo, una storia completa ipsius Studii, cioè dell'Università in quanto tale è quanto si ricava dalla prefazione di Parodi 1753, pp. [16], spec. p. [6], sotto forma di suppliche inviate al governo per chiedere sussidi, ottenuti in misura molto limitata. Sul Parodi, professore di Pandette - cioè di Diritto romano in prospettiva storica - vd. le note biografiche raccolte da Cesare Prelini, ristampate con aggiunte in Franchi 1925, pp. 357-362.

211 Trascrizione degli Acta: ASPv, Università, Studium Ticinense, cartt. 29-30; ivi, cart. 21-22 i Rotuli mss., dal 1374 al 1761; Syllabus Lectorum Studii Ticinensis, in ordine alfabetico (A-H; I-Z), che rappresenta la progettata «Litteraria Historia singulorum Lectorum»: ivi, cartt. 17-18.

212 Mazzilli Savini 2011; per la data, Erba 2011, spec. pp. 64-66.

213 Sul Bossi e il Beretti, vd. supra. Sul Villa e Comi, vd. infra. 
Il Parodi e i suoi antesignani avevano saputo distogliere lo sguardo dall'origo, per volgerlo finalmente alla progressio, cioè alla reale storia dopo il 1361. Del resto, il momento era maturo: i Fasti Gymnasii Patavini del Facciolati sono del 1757 e l'opera del Sarti De claris Archygimnasii Bononiensis professoribus a saec. XI usque ad saec. XIV uscirà fra il 1769 e 1772. Ma erano anche i tempi della politica, e non solo della cultura, a sollecitare il cambio di prospettiva. L'iniziativa del Parodi, in particolare, seguiva un'esortazione del presidente del Senato di Milano. Questa premura archivistica, di cui si hanno sentori almeno dal 1730, si manifesta verso il $1747-48^{214}$ e dunque precede l'incarico di riforma dell'Università affidato (e poi tolto) da Maria Teresa al Senato stesso nel 1753. ${ }^{215}$ Di sicuro, il lungo cammino della riforma asburgica dovette stimolare molti scandagli nella storia dell'Ateneo, sia a scopo informativo, sia per difendere l'esistente. Non mancò chi ricorse proprio al Capitolare di Lotario allora da poco edito dal Muratori, come alla prima di una serie di norme giuridiche ancora capace di fondare la pretesa pavese a essere centro di insegnamento: ${ }^{216}$ un uso che prefigura per molti versi l'impiego che del Capitolare sarà fatto nel 1925 in occasione della celebrazione degli undici secoli dalla presunta fondazione dell'Università, che - come vedremo - assumerà proprio la norma di Lotario dell' 825 come punto di riferimento.

Per tornare al Settecento, il rinnovato interesse per la storia (che nasceva all'incrocio fra l'indagine amministrativa e la rivendicazione di primato) era consono allo spirito con cui, a riforma anche edilizia realizzata, si disposero le lapidi dei maestri. D'altra parte, non era quella la prima volta che l'edificio universitario veniva adornato con le effigi dei grandi spiriti. Nel 1783, Siro Comi ricordava che in precedenza sulla facciata dell'Università («in scolastici huius theatri fron-

214 Parodi 1753, pref. n.num; cfr. la data delle due suppliche.

215 Sull'iniziativa del 1730, vd. Peroni 1925, pp. 123-134; sulla fase successiva Galeotti 1978; Zorzoli 1995, pp. 427-481.

216 Così Francesco Caimi nella memoria rivolta (forse del 1768) a Maria Teresa d'Austria per scongiurare il trasferimento dell'Università a Milano, di cui si vociferava (BUPv, Misc. Ticinensia, I 16). Sulla voce del possibile spostamento vd. per tutti Peroni 1925 , pp. 135 e nt. 2 e soprattutto Visconti 1925, pp. 209-216, che cita un documento del 1768 (apparentemente in italiano) che coincide largamente con quello latino firmato dal Caimi. 
te» $)^{217}$ erano stati dipinti personaggi come Baldo degli Ubaldi, Filippo Decio, Giasone del Maino, Raffaele Fulgosio, Giacomo Menochio. ${ }^{218}$ Proprio ragionando su questa galleria s'era accesa una disputa, cioè se fosse corretto includervi anche il ritratto di Francesco Filelfo: che il grande umanista di Tolentino fosse stato per un certo periodo di tempo al servizio di Filippo Maria Visconti nessuno dubitava, ma che avesse insegnato a Pavia era da dimostrare. ${ }^{219}$

All'impresa s'accinse appunto il Comi, allora poco più che quarantenne. ${ }^{220}$ Nato a Pavia nel 1741, da famiglia modesta, aveva seguito le lezioni di Siro Severino Capsoni al Collegio San Carlo, e ne aveva assorbito l'interesse per gli studi eruditi. Dopo avere conseguito la licenza, cioè il grado universitario di primo livello che consentiva l'esercizio della professione di notaio (1772), e avere assaporato un discreto successo come autore di componimenti poetici nell'Accademia degli Affidati, con il Franciscus Philelphus Archigymnasio Ticinensi vindicatus, dedicato nel 1783 a Johann Joseph von Wilzeck (1738-1819) da poco plenipotenziario, il Comi faceva il suo ingresso nel campo dell'erudizione municipale. ${ }^{221}$

Benché l'opera del Comi possa apparire, in ragione del tema prescelto, un passo avanti sulla via della storicizzazione, nella sua stessa struttura mostra visivamente con quanta fatica la nuova sensibilità sbocciasse dal corpo della vecchia o - rovesciando i termini - quanto la questione dell'origo continuasse ad annidarsi al centro della progressio. La docenza pavese del Filelfo è infatti quasi un pretesto, la

217 Comi 1783, p. 9.

218 Si potrebbe ipotizzare che queste immagini fossero le stesse dipinte nel 1765 in occasione della visita a Pavia di Maria Luigia, quando sulla facciata «tra l'una e l'altra delle finestre si effigiarono altrettanti busti di statura maggiore della naturale rappresentanti li più insigni maestri, che illustrata aveano la stessa Università»: il brano, del Giardini, è citato da Erba 2011, p. 58.

219 Nel merito, Maiocchi 1900 , p. 17, che recupera un documento (trascritto già dal Parodi) dell'8 novembre 1441 (ora in Codice diplomatico, II.2, doc. 571, pp. 436-437) in cui Filippo Visconti spiega che il Filelfo «nequaquam sit ad legendum in Studio illo constrictus». L'effettiva docenza del Filelfo è ora dimostrata da Cortesi 2012, p. 535.

220 Su di lui Bossi 1822; De Bernardi 1936, pp. 35-62; Cabrini 1982, pp. $565-$ 567; Regali 1999, pp. 167-178; Ead. 2000, pp. 401-405.

221 Comi 1783. Un esemplare interfogliato e postillato dal Comi è segnalato da Regali 1999, p. 170, nt. 13. 
stretta cornice in cui è incastonata una ben più ampia discussione sull'origine carolingia dello Studium ${ }^{222}$ che oppone il Comi ad Angelo Teodoro Villa, titolare della cattedra di Eloquenza greca e latina e di Storia italiana e lombarda, dalla quale avrebbe professato fino alla morte nel 1794.223

Nato a Milano nel 1723, attento alla poesia sia come autore sia come traduttore e antologizzatore in collaborazione con Filippo Argelati, il Villa nel Prodromus uscito nel 1782 non aveva fatto molto più che ampliare in un elegante latino le critiche mosse contro il libro di Antonio Gatti dal Muratori e soprattutto quelle rilanciate dal Tiraboschi dieci anni prima. Le tracce di scuole altomedievali - ripete il Villa - in nessun modo possono essere poste in continuità con lo Studium visconteo. Erano scuole ecclesiastiche e da cui esulavano molte materie, come l'eloquenza, la Filosofia, il Diritto, la Medicina: una limitazione alla formazione culturale del clero che il Villa sentiva di dover biasimare. ${ }^{224}$

Gli argomenti pubblicati dal Comi ${ }^{225}$ l'anno successivo sono ovviamente speculari, aderenti a quelli del Gatti, corroborati con un surplus di ragionamento sugli studi giuridici, che a suo dire erano praticati a Pavia almeno dal IX secolo. ${ }^{226}$ Comi valorizzava anche la notizia data da Roberto di Torigni (ca. 1110-1186), il monaco cronista che a Bec aveva risieduto non molti decenni dopo Lanfranco: un'aggiunta alla sua cronaca, sotto l'anno 1032, attribuisce la «inventio» delle leggi romane giustinianee «apud Bononiam» a «Lanfrancus Papiensis» e al suo «socius Guarnerius», i quali avrebbero poi anche insegnato il diritto giustinianeo. ${ }^{227}$ Secondo il Comi, Lanfranco tenne questo insegnamento a

${ }^{222} \mathrm{La}$ digresso sull'origo carolingia occupa oltre tre quarti dell'opera: Comi 1783, pp. 23-177.

223 Su di lui, Gabba - Magnino 1994, pp. 297-315; Tongiorgi 1997, pp. 29-48.

224 Sulla natura delle scuole in età carolingia, vd. il sempre valido Bullough 1964 , pp. 23-46, che propende per la natura ecclesiastica; vd. anche Esch 2006, pp. 709. 740 e Gavinelli 2012.

225 Regali 1999, p. 170.

226 Lo desumeva, ad esempio, dalla costituzione di Lotario sui feudi, emanata «per laudamentum sapientium», in cui il Comi vede giureconsulti attivi (anche) a Pavia. La attribuzione della costituzione a Lotario I (come vorrebbe la menzione di papa Eugenio II) è molto discussa: il testo è edito in MGH Const. 1 (1893), pp. 680-681, e viene lasciata aperta l'ipotesi della falsificazione.

227 Roberti de Monte, Cronica, MGH SS 6 (1844), p. 478: «Lanfrancus Papiensis 
Pavia. ${ }^{228}$ Siccome il Guarnerius che Roberto di Torigni indica come socius (che qui potrebbe voler dire allievo) di Lanfranco è da identificare con Irnerio, il maestro che illuminò - dopo Pepo - l'insegnamento bolognese, la notizia può diventare cruciale per stabilire addirittura la priorità di Pavia anche sul piano dell'insegnamento giuridico. ${ }^{229}$

La tela stesa dal Gatti iniziava insomma a riempirsi di dettagli. I Visconti - concludeva il Comi - non fecero che rifondare uno Studium che già era attivo dall'età di Carlo Magno e, dopo un declino, aveva conosciuto una ripresa nel secolo XI e specialmente nel XII e XIII in campo giuridico, per poi di nuovo declinare, specialmente a causa della lotta fra guelfi e ghibellini. A proposito della decisione di Galeazzo II, Comi aggiungeva che era stata presa «hortante, ut multorum opinio est, Francisco Petrarcha». Un'opinione sfornita d'ogni riscontro, ma che è tutt'ora spesso ripetuta; uno di quei luoghi comuni contro i quali la storia della storiografia può servire da antidoto. ${ }^{230}$

Sul piano del metodo la contrapposizione fra il Villa e il Comi celava, in definitiva, posizioni meno distanti di quel che possa apparire dalle divergenti conclusioni; intorno al mito d'origine si giocava una partita accesa forse più da rivalità professionali, in cui era coinvolto anche il maestro del Comi, il frate domenicano Siro Severino Capsoni (che a sua volta s'era pronunciato a favore del mito carolingio nel 1769). ${ }^{231}$

et Garnerius socius eius, repertis apud Bononiam legibus Romanis [...] operam dederant eas legere et aliis exponere. Sed Guarnerius in hoc perseveravit; Lanfrancus vero disciplinas liberales et literas divinas in Galliis multos edocens, tandem Beccum venit et ibi monachus factus est» (trad.: «Lanfranco di Pavia e Garnerius, suo allievo, ritrovate presso Bologna le leggi romane [...] iniziarono a leggerle e a esporle ad altri. $\mathrm{Ma}$ Guarnerius perseverò in questo; Lanfranco, invece, insegnando a molti, in Gallia, le arti liberali e le Sacre Scritture, venne infine a Bec e qui si fece monaco»).

228 Comi 1783, pp. 93-99, appoggiandosi anche alla testimonianza di Milo Crispinus (Lanfranci archiepiscopi Cantuariensis vita, in Patr. lat., 150, c. 29), contemporaneo di Lanfranco (su cui vd. Padoa Schioppa 2012, pp. 153-154). Il Comi richiama per quest'interpretazione Gatti 1704 , p. $91=1722$, p. 28 , che tuttavia non pare abbia utilizzato la testimonianza di Roberto di Torigni.

229 Il passo accomuna personaggi di generazioni diverse, ma in una prospettiva narrativa che non implica, da parte del cronista, l'idea che la loro azione fosse contemporanea: vd. approfonditamente Padovani 2007, pp. 71-140.

230 Ne faceva già giustizia Robolini 1836, V, 2, pp. 30-31.

231 Capsoni 1769, p. XLI. Sul Capsoni (1735-1796), vd. De Bernardi 1936, pp. 1033; in particolare pp. 17-22 per i rapporti epistolari con il Tiraboschi; Cabrini 1976, pp. 242-244; Repossi 1995, pp. 738-742; Gabba 2000, pp. 29-31; Milani 2000, pp. 417-420. 
Al Villa infatti il governo aveva commissionato fin dal 1778 una storia dell'Università, di cui nel Prodromus del 1782 dava i lineamenti: una ragionata esposizione dei fatti culturali e organizzativi dello Studio non prima, ma dopo Galeazzo II (con attenzione per l'ultimo quindicennio, «il più ricco di tutti per l'abbondanza e la grandezza dei fatti da raccontare» grazie allo sforzo riformatore degli Asburgo). ${ }^{232}$ L'incarico non doveva avere entusiasmato gli eruditi locali, e provocò una levata di scudi: il Villa - lo dichiara egli stesso - era stato avvertito che se avesse toccato il mito di Carlo Magno «sarebbe stato trafitto da più di trenta scritti». ${ }^{233} \mathrm{E}$ quando il Capsoni - che nel 1782 ottenne a sua volta dal governo, a gravare sulle entrate civiche, un contributo per il primo volume delle Memorie istoriche pavesi - si definiva «semplice collettor di memorie, non eloquente né sublime istorico», si contrapponeva evidentemente al Villa. ${ }^{234}$

Il Villa non riuscì tuttavia a portare a termine l'opera «la quale ebbe comprensibilmente a sfogarsi - mi occupa tutto, e mi costa tanta fatica, quanta nient'altro mai, dacché son al mondo». Compilò tuttavia quattro saggi di «storia delle discipline», dedicati alla Teologia, al Diritto canonico, alla Medicina e alla Fisica, insieme a una risposta ai due «Avversarij»: saggi che tuttavia non soddisfecero i committenti e andarono ad aggiungersi al limbo dei tentativi sfumati. ${ }^{235}$

Divisi sul momento, i protagonisti di questa tenzone erano in fondo avviati sul medesimo cammino, tracciato dall'uso critico delle fonti. $\grave{E}$

232 Villa 1782, p. 133: «copia tamen haec ipsa, et magnitudine rerum dicendarum longe erit locupletior». Il programma è esposto ivi, pp. 120-134: avrebbe dovuto riprodurre gli Statuti e le successive disposizioni, quindi l'elenco cronologico dei rettori, la prassi e le sue trasformazioni; i Collegi («Curotrophia»); infine, parte principale, una storia letteraria dei docenti.

233 Villa 1782, p. 5: «nisi institutum a Carolo M. hoc Ticinense Gymnasium tuearis, scito te scriptis plus quam triginta confodiendum esse»; la "minaccia" è già denunciata in Id. 1779, pp. VI-IX.

234 Un velenoso strale è in Capsoni 1785, II, p. IX, nt. 15: l'autore anonimo rimproverato per avere confuso Lanfranco di Canterbury con Lanfranco Beccari è il Villa come si può ricavare da Comi 1783, p. 74.

235 Il decorso dell'incarico è nelle cinque lettere del Villa edite in Memorie e Documenti, III, pp. 408-415, scritte al governo fra il 1779 e il 1791 (non 1793, come detto ivi, p. 413, nt. 1: 22 anni di servizio del Villa sono da calcolare a partire dal 1769; per la data della cattedra vd. Tongiorgi 1997, p. 37). Risulta che il Villa avesse ricevuto «quella parte dei manoscritti di Girolamo Bossi, che contiene notizie intorno allo Studio di Pavia»: ivi, p. 408. 
emblematico che nel 1804, un quarto di secolo più tardi del Villa, il Comi sia stato a sua volta invitato dal rettore Antonio Scarpa a comporre una storia dell'Università, a margine dell'incarico che lo stesso Comi s'era assunto di costituire un archivio dei documenti universitari. ${ }^{236}$

\section{IL RITORNO DEI LONGOBARDI ALL'EPOCA DEI NAZIONALISMI: Johannes MERKEl E PAVIA PRIMA Di BOLOGNA}

All'alba del XIX secolo uno dei motivi preferiti dal Gatti - Carlo Magno che chiama a Parigi maestri italiani - si prestava bene a essere rinverdito in un panegirico rivolto a Napoleone, che aveva chiamato a sé vari docenti pavesi, dall'Oriani al Mascheroni a Gregorio Fontana. Glielo indirizzava nel 1808 in bel latino il padre Stanislao Perondoli, emerito professore di Diritto canonico che elogiava il re d'Italia «vindice e restauratore delle scienze e delle lettere» e protettore dell'Università «sulle orme di Carlo Magno».237

Se a uso encomiastico il mito carolingio aveva attraversato indenne i dibattiti del Settecento, sul piano della critica storica, invece, la palma della vittoria sembrava dovesse ormai toccare al Muratori, al Tiraboschi e al Villa. ${ }^{238}$ Mentre Pavia e la sua Università passavano di

236 BUPv, Ticinesi, 439, cc. 160-217: Carte concernenti l'impiego di Archivista dell'Università, con il commento di Regali 1999, pp. 172-175; cfr. Cabrini 1982, pp. 565-567. Per le vicende dell'archivio successive al 1860, Negruzzo 2001, pp. 75-81. Il Comi possedeva le carte (dal 1361 al 1752) che erano state raccolte dal Parodi per il suo Elenchus. La storia non vide la luce, ma sopravvivono alcuni manoscritti di lavori preparatori, come Professores Gymnasii Ticinensis quorum nomina in Syllabo Parodiano desiderantur, che fu anche usato dal Prelini per Memorie e Documenti, I, p. 24 (cfr. BUPv, Ticinesi, 67; Regali 1999, p. 172, nt. 33).

237 Perondoli 1808, spec. pp. 33-36. È figura per altri versi interessante: Mantovani 2010, p. 296, nt. 63.

238 Vd. ad esempio Sangiorgio 1831, pp. 23-29, che critica esplicitamente il Gatti, sulle orme del Tiraboschi. Il Sangiorgio, medico, concede in proporzione maggior rilievo al Capitolare di Lotario, segno di un'attenzione che la figura di Dúngal e le sue esplorazioni culturali sempre stimolano in chi è attratto dai fatti scientifici più che da quelli istituzionali. Più sfumato Robolini (1823, I, 2, pp. 19-20 e pp. 26-27; p. 114), il quale, appoggiandosi al Comi, crede all'esistenza in età carolingia di una Scuola pubblica, ma pensa che dal 1225 fino al 1361 si interrompa ogni notizia di attività di uno Studium; cfr. Id. 1836, V, 2, p. 27. Il lavoro del Robolini sulla storia universitaria, soprattutto a partire dagli elenchi del Parodi, è testimoniato da BUPv, Ticinesi, 52; 274 e 726. 
nuovo di mano, dai francesi agli austriaci, nel 1815, Friedrich Carl von Savigny (1779-1861) pubblicava il primo volume della Geschichte des Römischen Rechts im Mittelalter. L'opera poneva su nuove basi anche lo studio storico delle Università. I racconti di fondazione erano esclusi dal perimetro e con essi il mito di Pavia prima Università d'Europa: la storia autentica, per il Savigny, comincia con Parigi e Bologna (con un posto a parte per Salerno). ${ }^{239}$

A Pavia è dedicato un trafiletto, come sede che, sorta nel 1361, non aveva fatto che seguire modelli consolidati, seppure con un suo sviluppo prestigioso. L'opera del Gatti, di conseguenza, è giudicata «poco istruttiva»; non inutile, invece, il regesto dei documenti allestito dal Parodi. ${ }^{240}$ Non si potrebbe trovare distinzione più nitida fra le due possibili direzioni della storiografia, quella che abbiamo chiamato anamnestica e quella analitica.

Eppure, proprio dalla scuola del Savigny, doveva presto venire un nuovo impulso a ritornare alla storia anteriore al 1361. Solo che la fabula entrava, per così dire, nell'bistoria.

L'innesco fu la lettura compiuta nel 1820-21 a Vienna, da parte del giovane Georg Heinrich Pertz (1795-1876), di un manoscritto che presentava le leggi longobarde e i capitolari dei re franchi e sassoni in un ordine diverso da quello dei codici fino ad allora conosciuti (ad esempio, diverso dall'ordine seguito dal «Codice delle Leggi» modenese dal quale il Muratori aveva tratto il Capitolare di Lotario dell'825). ${ }^{241}$

La ricerca di altri manoscritti, il confronto con quelli già noti e una complessiva rimeditazione in vista dell'edizione di tutto il materiale determinarono la rifondazione degli studi sul diritto longobardo, che prese la forma di un breve, ma decisivo scritto proprio di un allievo del Savigny, Johannes Merkel (1819-1861), al quale il Pertz aveva affidato

239 Cito dalla seconda edizione: Savigny 1834, III, cap. XXI $₫ 59$, p. 156.

240 Ivi $\$ 125$, pp. 335-336 (risp. «wenig lehrreich» e «nicht ohne manche brauchbare Notizien»).

241 Una prima raccolta dell'Edictus [sic] longobardo s'accumulò con le leggi emanate sino ad Astolfo. Con variazioni nell'ordine, in base alla materia, la normativa longobarda confluì insieme alle norme delle principali genti presenti nel Sacro Romano Impero nella raccolta compiuta nella prima metà del IX secolo da Lupo di Ferrières, testimoniata dal ms. Modena, Archivio Capitolare, O.I.2 (la cui scrittura è oggi datata alla seconda metà del IX secolo): cfr. Merkel 1850, pp. 18-19; Bluhme (MGH LL 4 [1868], pp. XL-XLII) e i progressi della ricerca in Golinelli 2008, pp. 17-35. 
il compito dell'edizione, insieme a Friedrich Bluhme (1797-1874), altro grande ingegno cresciuto alla scuola di Gustav Hugo e del Savigny.

L'opuscolo preparatorio del Merkel - allora trentenne - apparve nel 1850 come «contributo» alla Geschichte del Savigny; il primo dei tre capitoli di cui si componeva, intitolato significativamente Die Rechtsschule zu Pavia, si apre con questo enunciato:

La scuola giuridica di Pavia risale all'epoca di Ottone I, e senza dubbio è derivata dalla più antica scuola di grammatica, le cui tracce si osservano dal settimo secolo in poi. ${ }^{242}$

Questa breve frase, a metà del XIX secolo, condensa il passato e il presente della storiografia sulle origini dello Studium. Da una parte, Merkel accredita la presenza ininterrotta a Pavia di una scuola di grammatica (il VII secolo allude verosimilmente al grammatico Felice ricordato da Paolo Diacono e attivo presso la corte di Cuniperto); ${ }^{243}$ dall'altra, stabilisce l'esistenza di una scuola giuridica dalla metà del X secolo (Ottone occupa Pavia e assume il titolo di re d'Italia nel 951). Infine, ma non ultimo, Merkel lega fra loro le due "scuole", configurando quella giuridica come una diramazione di quella grammaticale. Così facendo, porta a compimento e riapre le ricerche sulle origini, che in varie forme avevano caparbiamente predicato l'esistenza di un centro di studi a Pavia fin dall'alto Medioevo: supplisce con una documentazione di nuovo tipo (giuridico) alla scarsità di notizie sul versante retoricogrammaticale successive all'età carolingia; offre uno sfondo per meglio situare la formazione, nell'XI secolo, di una personalità chiave come Lanfranco di Pavia, in cui si mischiano arti liberali e diritto; costruisce la base per rivendicare il primato di Pavia rispetto a Bologna anche sul piano della giurisprudenza.

Secondo Merkel, gli «organi» della «Rechtsschule zu Pavia» erano «insieme maestri e giudici, teorici e pratici». A loro si deve la raccolta in un unico "codice" degli editti longobardi e dei capitolari emanati a partire da Carlo il Grosso, nella forma che era apparsa nel manoscritto viennese scoperto dal Pertz e confermata da altri testimoni: libro che pertan-

242 Merkel 1850, p. 13: «Die Rechtsschule zu Pavia reicht hinauf bis in Ottos I Zeit, und ist unbezweifelt aus der älteren grammatischen Schule hervorgegangen, deren Spuren von siebenten Jahrhundert an beobachtet werden».

243 Su di lui, vd. il compianto Radiciotti 1996, pp. 25-26. 
to Merkel battezzò Liber Papiensis. Ai maestri pavesi risale anche la stesura di formule per facilitare l'applicazione delle norme stesse (il Cartularium Langobardicum). Soprattutto, essi lasciarono un commento (Merkel parla di «glossa», alludendo volutamente alla forma che sarà poi tipica dei maestri bolognesi) alla legislazione longobarda, carolingia e sassone, che si distingue anche per la finezza precorritrice con cui attinge al diritto romano: «Tutto questo - conclude Merkel - è accaduto prima che Bologna fosse citata come scuola giuridica, anzi è dimostrabile che ciò sia in parte avvenuto più di cento anni prima».244

Il commento fu battezzato Expositio ad Librum Papiensem: tramandato da un manoscritto conservato a Napoli - il codice Brancacciano - riporta in vita nomi e personalità di giuristi impegnati in discussioni complesse, in cui le norme longobarde sono esaminate con l'ausilio della dialettica e del diritto romano. ${ }^{245}$ Fra essi compare Lanfranco archiepiscopus, il personaggio che da Pavia passò a Bec, poi a Caen e di qui in Britannia. Non è l'unica, straordinaria presenza che leghi l'Expositio a Pavia. Altri dei giuristi che vi sono citati, come Bonfiglio e Guglielmo, sono attestati in vari documenti coevi come iudices sacri palatii presenti a Pavia; anche l'ambientazione dei casi fa spesso riferimento a Pavia, come quello che presenta una ragazza che lava le vesti in Ticino. ${ }^{246}$ Sono questi elementi, tratti dai testi, che permettono al Merkel di riportare in vita la scuola di Pavia, che a suo parere sorse al tempo di Ottone I, che aveva occupato Pavia nel 951, e raggiunse il culmine nella prima metà dell'XI secolo.

In realtà, la sua abbagliante dimostrazione lasciava vari punti in ombra, sia verso il passato sia verso il futuro. All'indietro, resta imprecisato il collegamento fra la scuola giuridica palatina di X-XI secolo e la precedente scuola grammaticale d'età carolingia; in avanti, non è affrontato il rapporto (se vi fu) fra la scuola giuridica palatina e lo Studium del 1361.

Dal punto di vista della storia della storiografia - che è quello che

244 Merkel 1850, p. 13

245 Napoli - Bibl. Nazionale, ms. Branc. I.B.12 (antea II.B.28). L'autore dell'Expositio si rifaceva non al Liber Papiensis (tenuto presente dai giuristi "pavesi"), ma alla cd. Lombarda: vd. Bluhme, in MGH LL 4 [1868], p. CII, con le precisazioni di Padoa Schioppa 2012, pp. 143-164.

246 Exp. ad Liut. 134 (MGH LL 4 [1868], p. 466). Per la documentazione aggiornata sui iudices, vd. ora Radding 1988, pp. 94-95. 
qui adottiamo, mentre spetta ad altri discutere il problema storico ${ }^{247}-$ un aspetto colpisce nella ricostruzione del Merkel, ossia la coincidenza fra l'esaltazione di Pavia come prima scuola giuridica e la celebrazione dello «spirito germanico» cui si deve questa rinascita giuridica: «Infatti - scrive Merkel - il tribunale palatino dell'imperatore tedesco è divenuta la Facoltà giuridica di Pavia».248

È una manifestazione del nazionalismo che permeava, in quei decenni, la storia del diritto in Germania e aveva portato al sorgere di due correnti, quella dei romanisti e quella dei germanisti: gli uni - nella scia di Savigny - davano rilievo al ruolo giocato dal diritto romano (e dai giuristi) nell'elaborazione medievale del diritto tedesco, gli altri valorizzavano gli elementi consuetudinari e pratici, e più direttamente popolari. ${ }^{249}$ Merkel, trentenne allievo di Savigny - verso cui dichiara tutto il proprio debito intellettuale $e^{250}$ - trova in un certo senso una conciliazione: il merito di avere riavviato la cultura giuridica europea sulle basi del diritto romano toccava non a Bologna, ma a un ambiente che, seppure situato in Italia, ruotava intorno agli imperatori sassoni e aveva come sostrato le norme dei popoli nordici che, a partire dai Longobardi, avevano via via occupato l'Italia e fatto di Pavia la loro capitale..$^{251}$

Una esplicita coloritura nazionalistica permeava dunque l'inter-

247 Vd. Padoa Schioppa 2012, pp. 143-164.

248 Merkel 1850, p. 16: «Auf solche Beweise gestützt kann ich die ersten Anfänge des Rechtsstudiums im Mittelalter [...] auf die Grundlagen eines germanisches Rechts und was noch wichtiger ist auf die Thaten des germanischen Geistes zurückführen. Denn das Pfalzgericht des deutschen Kaisers ist die Juristenfacultät zu Pavia gewesen». Precisa poi (ivi, p. 28) che fra fine X e inizio XI secolo vivevano a Pavia giudici palatini, le cui sentenze acquisirono un particolare valore come interpretazione delle norme, tale da imporsi sugli altri giudici; perciò «dal centro di un tribunale supremo tedesco è stata fondata nel regno italico la teoria del diritto comune e del processo comune, e soprattutto messa per iscritto e raccolta».

249 Sulle due scuole, importante Conte 2009, pp. 13-42; è un riflesso simmetrico "la questione longobardica" suscitata dal Manzoni, se cioè i Longobardi si fossero o meno fusi con i Romani conquistati (supra, nt. 80).

${ }^{250}$ L'opera è a lui dedicata, e una lunga premessa dichiara la devozione di Merkel 1850, s.n.p.

251 Le opposte motivazioni ideologiche sono bene evidenziate da Mengozzi 1924, pp. 354-357. Esplicitamente dedicato a contestare al Merkel latendenza a «germanizzare» la scuola di Pavia è il saggio di Bassi Costa 1951, spec. pp. 5-6; 40-43, che riconduce l'origine di tutti gli sviluppi didattici e culturali alla «scuola vescovile pavese». 
pretazione di Merkel, che si saldava - per dire così involontariamente con la lunga aspirazione pavese a rivendicare un primato universitario europeo. Quando il Merkel pubblicava il suo saggio, da pochi mesi s'era conclusa la Prima guerra d'Indipendenza, combattuta anche intorno a Pavia; gli studenti erano sempre più insofferenti del regime austriaco, che proponeva fra l'altro una riforma degli studi giuridici che accentuava l'omogeneità con il curriculum di Vienna. ${ }^{252}$ Sulle vie della ricerca erudita, dalla Prussia - che nel 1848 aveva celebrato la sua rivoluzione e si apprestava a scontrarsi a sua volta con gli Asburgo - veniva invece nuovo alimento all'antico sogno del primato pavese.

Non si tratta solo di sottolineare un'ambientazione. Il nesso che, anche nella ricerca successiva, è sembrato non completamente provato - tra iudices all'opera sulla legislazione longobarda e la presenza di una scuola intesa come struttura dedicata all'insegnamento ${ }^{253}$ - ci appare forgiato, dal suo primo artefice, con un sovrappiù ideologico, il che può spiegarne la debolezza. Esporre la ricostruzione del Merkel alla luce dell'ideologia lascia trasparire un secondo aspetto: la connessione fra i giuristi pavesi e il palazzo, su cui insiste, conferisce alla scuola - «la Facoltà giuridica di Pavia» - tratti istituzionali che la assimilano implicitamente a una Università. Anche se il Merkel si disinteressava della questione, la sua interpretazione e il ruolo assegnato a Ottone I prefiguravano perciò la linea continuista con lo Studium del 1361: in fondo, anche lo Studium visconteo fu fondato con un privilegio di un imperatore del Sacro Romano Impero.

I risultati del Merkel furono entusiasticamente accolti da Alessandro Nova (1819-1887), docente di Filosofia noto specialmente per la sua polemica con il Rosmini in difesa dell'utilitarismo di Romagnosi, che gli valse qualche ironia. ${ }^{254} \mathrm{Nel}$ «discorso letto nella solenne inaugurazione dell'insegnamento universitario in Pavia il 20

252 Egglmaier 1997, pp. 107-118.

253 Altro è parlare in senso metaforico di una "scuola pavese di Diritto longobardo", intendendo con ciò una semplice tradizione interpretativa sorta e tramandata al di fuori di una scuola intesa come centro deputato all'insegnamento. L'ambivalenza del termine è anch'essa foriera di ambiguità storiografiche.

254 Per la polemica, vd. Malusa 2011, p. 26, nt. 5; di Nova «inimicissimo di Rosmini» e amante «dell'olio di Merluzzo» lascia un bozzetto d'alunno Carlo Dossi (2010, nr. 460, p. 27; nr. 2498, p. 238; nr. 2533, p. 249). Nova insegnò Filosofia e Storia della filosofia dal 1854; dal 1856 (anche) Diritto romano e Storia, dal 1859 
novembre 1859», il primo anno accademico dopo la liberazione della Lombardia, Nova si proponeva di dimostrare come all'istruzione siano indispensabili la «libertà e indipendenza nazionale, senza cui l'Università più doviziosa è superbo cadavere ${ }^{255} \mathrm{e}$, insieme, di difendere il diritto dell'Università di Pavia a essere mantenuta nella sua integrità, soprattutto contro il «divisamento di sbrancarne la facoltà filosofica», sancito dalla legge Casati che, giusto una settimana prima, aveva stabilito di spostarla a Milano. ${ }^{256}$

Fu per primo il Nova, in questa prolusione, a stabilire il collegamento fra le ricerche della scuola giuridica del Savigny e la tradizione erudita settecentesca capeggiata dal Gatti: «i nuovi documenti scoperti dal Merkel convalidano le sagaci osservazioni di Capsoni e Comi dirette a stabilire che altresì la coltura classica greco-latina in Pavia od era sempre continuata anche dopo le invasioni dei barbari, od era già risorta spontaneamente nel secolo $\mathrm{X} \gg,{ }^{257}$ con la conclusione che «Pavia adunque fu nel medio evo il primo centro della coltura scientifica cristiana nell'Europa occidentale». ${ }^{258}$

Il Nova s'incaricava di proseguire la linea oltre il punto in cui l'aveva lasciata il Merkel, congiungendo la scuola giuridica dell'XI secolo allo Studium trecentesco. Così, in particolare, il passo fondamentale dell'Azario «Dominus Galeaz curavit habere universa studia in civitate Papiae, in qua antiquitus fuisse dicuntur et certe de iure bene stat $\rangle^{259} \mathrm{fu}$ dal Nova forzato fino a fargli affermare che Galeazzo II «procacciò di possedere tutti gli Studii nella città di Pavia, nella quale dicesi, che fossero stati anticamente, e certamente lo studio del Diritto vi dura tuttora prosperevole», come se non si fosse mai interrotto dai tempi di Ottone I. Interpretazione spesso ripetuta dopo di lui, che trascura che

Diritto penale; fu rettore nel 1879-80: vd. il necrologio nell'Annuario 1887-88, pp. 5658; Memorie e Documenti, I, pp. 408; 490-491. Sul suo ruolo nella compilazione di quest'ultima raccolta, vd. infra.

255 Nova 1862, pp. 47-244; la citazione è tratta da p. 48.

256 Analogo richiamo ai risultati del Merkel, in funzione di rivendicare la reintegrazione a Pavia della Facoltà filosofica, è compiuto dal sacerdote Terenzio 1861, pp. 31-33. Per le vicende innescate dalla legge Casati, vd. Magnino 2000, pp. 475-478; Signori 2002, pp. 49-116.

257 Nova 1862, p. 69.

258 Ivi, p. 71.

259 Azario 1730, cap. XII, col. $406=1927$, p. 159. 
l'espressione «de iure» in Azario significa «a buon diritto, certamente» e non già «lo studio del Diritto» e «bene stat» si riferisce alle favorevoli condizioni logistiche offerte dalla città trecentesca. ${ }^{260}$

Mentre la storiografia pavese piantava la bandiera sui nuovi risultati, ${ }^{261}$ il Liber Papiensis, l'Expositio e il Cartularium Langobardicum furono editi nel 1868 da Alfred Boretius (1836-1900), che compì l'impresa preclusa al Merkel dalla morte prematura. ${ }^{262}$ L'interpretazione generale fu da lui confermata, con alcune precisazioni. ${ }^{263}$ Quanto all'Expositio, in particolare, gli pareva certo che fosse stata redatta a Pavia intorno al 1070, testimonianza di un momento culturale ancora notevole, poiché la qualità del commento mostra un autore «litteris imbutum, ingenio callidum, in iure bene eruditum, in legibus explicandis artis criticae minime ignarum» e già a conoscenza di tutte le parti del diritto giustinianeo, Digesto incluso. ${ }^{264}$ Soprattutto, anche per il Boretius l'attività di Lanfranco e degli altri giuristi citati nell'Expositio s'era svolta nell'ambito di una scuola situata a Pavia (a partire dall'an-

260 Il passo è oggetto di un'accanita discussione in Nova 1862, pp. 143-161, che non tocca tuttavia il punto fondamentale, ossia il significato della locuzione «de iure»: vd., nel significato di «a buon diritto, senza dubbio» (Azario 1730, coll. 389; 399). Vd. supra per il significato dell'espressione nel contesto, dove serve a spiegare le caratteristiche logistiche della città in virtù delle quali il nuovo Studium «vi sta bene». La dissertazione del Nova 1862, risp. pp. 165-195 e pp. 195-216) s'incentra poi sulla lettera del Monaco di San Vittore (già messa in luce dal Robolini 1830, IV, 1 p. 349) e sulle testimonianze di Milone Crispino e di Roberto di Torigni riguardo alla vita di Lanfranco (su cui vd. supra).

261 Le conclusioni del Nova furono ovviamente accolte con particolare entusiasmo dai giuristi; vd. ad es. un notevole amministrativista come de Gioannis Gianquinto 1864, pp. $75-76$.

262 Boretius, che fu deputato nazional-liberale al Reichstag e alla camera prussiana, soccombette a sua volta alla fatica dell'edizione dei Capitularia regum Francorum - di Carlo il Grosso e successori - che fu completata da Victor Krause (MGH Capit. 2 [1897], pp. IX-X). Su questi passaggi di testimone, esempi di dedizione alla ricerca, ma anche sulle tensioni fra studiosi - che portarono alla rottura insanabile fra Pertz e Boretius - vd. Bresslau 1921, pp. 439-445; 580-582; 680-682. Sui limiti dell'edizione, vd. Bougard 1995, pp. 17-20.

263 Sull'origine del Liber Papiensis, vd. Boretius, in MGH LL 4 (1868), p. LXII; sulle formule del libro di Walcausus, ivi, p. LXXXIV, con l'ipotesi che possa essere d'origine romana; sul Cartularium, ivi, pp. XCII-XCIII. A suo avviso i capitolari franchi e sassoni erano applicabili non solo ai Longobardi, ma a tutte le nazioni viventi congiuntamente nel regno Franco e Italico (ivi, p. LXIV).

264 MGH LL 4 (1868), pp. LXXXVII-XC; aveva scopo pratico: ivi, p. LXXXVII. 
no 1000), nella quale gli stessi individui fungevano da «legum magistri» e da «iudices» e in cui la giurisprudenza ancora era sentita come parte della retorica. ${ }^{265}$

A metà del XIX secolo, i Longobardi facevano dunque di nuovo irruzione nella questione delle origini dello Studium di Pavia: non più come fondatori della scuola, ma come re legislatori, le cui norme, insieme a quelle dei successivi sovrani, avevano innescato una scuola giuridica. Era il riflesso - paradossale solo negli esiti, ma non certo nel metodo - dei progressi compiuti dalla Scuola Storica del diritto inaugurata dal Savigny. Anche il versante delle indagini sulla vita dell'Università dopo il 1361 doveva a maggior ragione esserne investito. Come spesso capita, l'impulso fu dato da un evento celebrativo, ossia la «Mostra Regionale e Provinciale agricolo-industriale-didattica» che doveva tenersi nel settembre 1877, avendo come teatro i portici dell'Università: un momento in cui, nello slancio post-unitario e nel clima del positivismo, i tempi dell'attività economica e dell'Università si trovarono, per una volta, in sincronia.

In vista dell'avvenimento, il 5 dicembre 1876 il rettore Alfonso Corradi (1833-1892), costituì una «commissione universitaria per la pubblicazione di un lavoro storico intorno all'Università», di cui facevano parte, oltre al medesimo Corradi, che era farmacologo ${ }^{266}$ (e si impegnava per la «medicina») e al già citato Alessandro Nova (per «la giurisprudenza, la filosofia e le discipline Orientali»), Antonio Zoncada, ordinario di Letteratura italiana (per «la letteratura»), Carlo Magenta, di Storia moderna (per «la storia»), il numismatico e ispettore degli scavi e monumenti della provincia di Pavia Camillo Brambilla (per «i monumenti», tema cui chiese di associare Carlo Dell'Acqua), il fisico Eugenio Beltrami (per la «matematica, la fisica e la chimica e scienze naturali»), il sac. Cesare Prelinii ${ }^{267}$ (per le «indagini nell'Archivio

265 MGH LL 4 (1868), p. XCV, in particolare prendendo spunto dalla figura di uno degli interpreti menzionati dall'Expositio, Sigfrido. Sulla datazione, spostata un poco più avanti rispetto al Merkel, ivi, pp. XCIII-XCVI.

266 Vd. il necrologio (siglato S.) in Annuario 1892-93, pp. 89-92.

267 Sul ruolo del Prelini, incaricato fin dal 1875 di «riordinare l'archivio universitario», informa una dichiarazione del rettore Nova che il sacerdote si fece rilasciare «per valersene in un concorso al posto di Vicebibliotecario» (PAVIA - ARCHIVIO STORICO DELL'UnIVERSITÀ, ASUPv, Carteggio non schedato, Rettorato 1878-79: ivi, altri carteggi per richieste di documenti, su Scarpa e Spallanzani). L'incarico gli fu ufficialmente confermato con l'impegno di concluderlo entro un triennio, con decreto del 
come sussidio ai membri della Commissione») e il segretario dell'Ateneo, Vittorio Piccaroli (incaricato di collaborare con il Prelini al riordino dell'Archivio universitario). ${ }^{268}$ L'opera - come spiega bene il verbale della riunione fondativa ${ }^{269}$ - avrebbe dovuto «delineare la storia dell'Università mercè la notizia de' rettori e de' professori che ne salirono le cattedre».

Nonostante il «difetto di tempo», che si riduceva a pochi mesi, il piano fu alacremente eseguito e fra il 1877 e il ' 78 furono pubblicati i tre tomi di Memorie e Documenti per la storia dell'Università di Pavia e degli uomini più illustri che v'insegnarono. Gli autori si avvalsero largamente delle carte inedite di alcuni degli eruditi dei secoli precedenti, in particolare del Bossi e del Parodi per gli elenchi di rettori e docenti. ${ }^{270}$ L'assetto,

Ministro Giovanni Puccini, il 19 gennaio 1879, con retribuzione retroattiva dal marzo 1878 (ASUPv, Fascicoli personali, Cesare Prelini). Sul Prelini, notizie in Majocchi 2001, pp. 52-53; 80-83.

268 Sui fondi disponibili al 18 agosto 1878 e sui criteri di riordino informa una preziosa nota del Prelini al Rettore (ASUPv, Fascicoli personali, Cesare Prelini): «si riposero nell'ordine cronologico tutti gli atti riguardanti gli Esami e le Lauree delle varie Facoltà dal 1370 al presente, si riordinarono e rividero gli Archivi particolari delle Società dei Nob. Giurisperiti pavesi e degli Aromatari, che hanno un numero assai rilevante di carte; si rividdero e disposero in miglior ordine circa novanta cartelle di corrispondenza del Rettorato dal 1763 al 1818-19, il cui ordinamento, oltre a pochi altri atti, costò al Signor Siro Comi la fatica di quindici anni circa, con un aiutante, come appare dagli atti stessi. Rimane ora a formulare un Repertorio delle suddette cartelle, che manca affatto; sono già iniziati i Repertorii delle Lauree antiche di più che quattro secoli e degli Atti storici della Università. Rimane dopo questi a fare un materiale riordinamento della Facoltà filosofica, che non è ancora in consegna dello scrivente». L'assetto raggiunto dall'Archivio del Rettorato è descritto pochi anni dopo dal Maiocchi nel Codice diplomatico, I, p. 3. Vd. anche infra, nt. 270.

269 ASUPv, Archivio Generale, 4-14, Cerimonie-Congressi-Associazioni-Mostre ed esposizioni varie 1877 (qui anche i documenti preparatori dell'Esposizione, in occasione della quale, il 12 settembre fu restituito il «cimelio delle antiche porte di bronzo [...] che nelle antiche lotte intestine i Ravennati asportarono come trofeo di guerra»: ivi, il sindaco Arnaboldi al Rettore, 30 agosto 1877). Oggetto di discussione, suscitata dal Magenta, fu se ci si dovesse accontentare, in considerazione del difetto di tempo, di una «succinta storia» da premettere alla raccolta dei documenti; si pensava, ma non fu fatto, di pubblicare i Cenni redatti nel 1864 da Giovanni Maria Bussedi (conservati manoscritti in BUPv, Ticinesi, 212): una storia schematica, ma non priva di spunti (specialmente quanto ai tentativi di spostamento a Milano).

270 L'opera fu occasione di spostamenti di fondi d'archivio (ASUPV, Carteggio non schedato, Rettorato 1878-79): il 6 febbraio 1877, il rettore Corradi chiese al vescovo 
che dà molto spazio a schede bio-bibliografiche dedicate ai professori posteriori al 1753 (che ne costituiscono il contributo più originale), fa anzi dell'opera un'esplicita continuazione di quella bistoria litteraria che il Parodi aveva condotto fino a metà del Settecento nel suo Syllabus Lectorum rimasto inedito (che a sua volta riprendeva un'idea che era stata del Bossi e poi tante volte accarezzata, dal Baretti al Villa) ${ }^{271}$ La raccolta di Memorie e Documenti promossa dal Corradi può dunque essere considerata punto d'arrivo di oltre due secoli di preparazione; al contempo era in sintonia con il positivismo storiografico allora imperante, che richiedeva l'accertamento documentato dei fatti storici (spicca, d'altra parte, la dichiarata propensione degli estensori per l'introspezione psicologica dei personaggi dei quali offrono le biografie: propensione frutto forse del versante scientistico del positivismo). ${ }^{272}$

Nel quadro più generale di un rinnovamento della storiografia universitaria su scala europea - segnata in particolare dai trattati di Heinrich Denifle (1885) e di Hastings Rashdall (1895) - per Pavia l'Ottocento si chiude con l'edizione dello statuto dell'Universitas degli studenti giuristi del 1395, fortunosamente ritrovato in copia a Basilea. ${ }^{273}$ All'inizio del secolo seguente, si ispira esplicitamente al Chartularium Universitatis Parisiensis - pubblicato a partire dal 1889 dal p. Denifle e da Émile Chatelain - l'impresa del Codice diplomatico dell'Università di Pavia, pro-

Lucido Maria Parocchi di consegnare le tre cartelle contenenti gli Atti Cancellereschi relativi all'Università di Pavia, rimaste «per inavvertenza» presso la Curia vescovile nel trasferimento delle carte avvenuto alla fine del Settecento (s'intende quando vi mise mano il Comi): si tratta, se non erro, dei documenti notarili della cui consegna il 17 febbraio dà accurato conto Iaria 2007, pp. 109-110. Si chiedeva anche di potere trarre copia del repertorio del notaio Griffi (sul quale vd. Crotti - Majocchi 2005); cfr. Codice diplomatico, I, p. 3.

271 Fra i vari studiosi che ripresero il lavoro del Parodi, va segnalato qui Costantino Gianorini. Un esemplare interfogliato dell'Elenchus (in BUPv, Ticinesi, 90) reca ampie note ai singoli documenti regestati, che il Gianorini sembra aver per lo più ricavato dalla consultazione della trascrizione integrale dei documenti lasciata manoscritta dal Parodi. Soprattutto, il Gianorini trascrisse in schede il Syllabus lectorum del Parodi, con molte correzioni, integrazioni bio-bibliografiche (tratte specialmente da opere a stampa) e numerose aggiunte di docenti non rilevati dal Parodi (BUPv, Ticinesi, 527).

272 Corradi in Memorie e Documenti, I, p. VII: «procurammo altresì di addentrarci nella vita intima» (corsivo originale).

273 Hürbin 1898; da segnalare, ivi, p. 3, la piena accettazione dell'idea che Pavia possedesse una fiorente scuola giuridica prima di Bologna. 
mossa da Giacinto Romano (1854-1920) sotto l'egida della neonata «Società Pavese di Storia Patria» e compiuta fra il 1905 e il 1915 dal sacerdote Rodolfo Maiocchi (1862-1924), epigono di una serie di eruditi locali provenienti dal clero cittadino. ${ }^{274} \mathrm{Il}$ Maiocchi, constatando la perdita di molti dei documenti originali, si appoggiò in buona parte alle trascrizioni degli Acta Studii Ticinensis lasciate manoscritte dal Parodi, una volta di più miniera per le iniziative editoriali successive. ${ }^{275} \mathrm{Il}$ prezioso Codice diplomatico del Maiocchi perviene al 1450 (per il cinquantennio successivo ne rimane un abbozzo manoscritto). ${ }^{276} \mathrm{La}$ sua prosecuzione, corroborata da una ricognizione allargata anche agli archivi milanesi, è stata promossa negli scorsi anni '90 dal Centro per la storia dell'Università di Pavia, su impulso di Agostino Sottili (1939-2004), e procede nelle due serie delle Lauree e dei Documenti. ${ }^{277}$

\section{UNA DATA PER UNA CELEBRAZIONE: I SAECULARIA UNDECIMA E IL CAPITOLARE DI LOTARIO}

L'Annuario dell'Università di Pavia del 1899-1900, il primo edito nel nuovo secolo, si apre con Cenni storici intorno all'Università, anonimi, ma di Mariano Mariani (1838-1914), professore di Procedura civile fin dal 1863 , e solido cultore delle memorie locali. ${ }^{278} \mathrm{Il}$ breve scritto rispecchia fedelmente il nuovo clima storiografico.

274 Su di lui, vd. Soriga 1926, pp. 231-233 e Majocchi 2001, pp. 55-57. Sul Romano, Settia 2001, pp. 9-15.

275 Importante per ricostruire l'assetto documentale e la sorte dei vari fondi l'Avvertenza preliminare: Codice diplomatico, I, pp. 3-6 (ivi, p. 5 il riferimento al Chartularium di Parigi). secolo XV.

276 Pavia - Bibl. civica Bonetta, ms. II 39: Maiocchi, Documenti universitari del

277 Lauree: Sottili 1995 (aa. 1450-1475); Id. 1998 (aa. 1476-1490); Sottili - Iaria 2008 (aa. 1491-1499, con integrazioni aa. 1425-1482); Documenti: Sottili 1994 (aa. 1450-1455); Sottili - Rosso 2002 (aa. 1456-1460); Iaria 2010 (aa. 1461-1463). Per l'impulso alla continuazione del Codice diplomatico, vd. Sottili 1983, pp. 146-151. Va ricordato che per gli anni successivi al 1390 il Maiocchi, per brevità, offre solo uno «stringato regesto» delle lauree (Codice diplomatico, I, p. 4).

278 Il testo gli è attribuito nell'edizione autonoma: Mariani 1900. I Cenni sono provvisti di un'ottima bibliografia curata da Zanino Volta (che ha il merito di uno studio precursore sui documenti di laurea fino al 1423: Volta 1890, pp. 517-584). Oltre che 
Al Mariani, l'«opinione di un'origine antichissima» dello Studio pavese «sorta forse dalla gelosia e dallo spirito di rivalità proprio delle città italiane» appare definitivamente superata. Gli argomenti del Nova, ultimo alfiere di quell'opinione, sono passati in rassegna e rapidamente abbattuti: «La fondazione dello Studio ticinese è dovuta al popolo pavese» nel 1361. ${ }^{279}$ Anche se non immune da una qualche ideologia quando toglie ruolo ai Visconti per darlo alla città, il Mariani sembra chiudere definitivamente fuori dal nuovo secolo una discussione che era iniziata con lo Studium stesso. ${ }^{280}$

Il suo ragionamento, tuttavia, conteneva la profezia che l'avrebbe smentito: lo spirito di rivalità, cui il Mariani imputava la genesi del mito, era tutt'altro che estinto e avrebbe potuto fare risorgere la querelle.

Trascorsi solo 25 anni, lo stesso Annuario si apre con un discorso del rettore Arrigo Solmi, che dichiara il 1925 l'anno in cui l'Università avrebbe celebrato gli «undici secoli dal remoto anno 825 dell'era nostra, in cui una prima riforma degli studi designava Pavia a sede e centro dell'insegnamento dottrinale, chiamandovi i docenti più famosi e dando così il primo impulso all'organizzazione delle scuole superiori dell'Occidente».

L'emiliano Arrigo Solmi (1873-1944) era uno dei maggiori storici del diritto del momento, specialista dell'età medievale, e politico emergente; vicino ai gruppi nazional-liberali, deputato eletto nella «lista nazionale», avrebbe aderito al PNF nel 1925 e sarebbe divenuto nel 1932 sottosegretario all'Educazione Nazionale, quindi guardasigilli dal 1935 fino al 1939. Era rettore dal novembre 1923 e lo sarebbe stato fino al gennaio 1926, al termine delle celebrazioni. ${ }^{281}$

In uno studioso di vaglia come il Solmi - fra l'altro editore e primo vero interprete delle Honorantie civitatis Papie ${ }^{282}$ - le sfumature sono difficilmente casuali. Così, quando affermava che, con il

autore di vari saggi sulla storia universitaria e consigliere della Società Pavese di Storia Patria, Mariani ebbe un ruolo nella costituzione della collezione numismatica dei Musei Civici, vd. Ottocento e Novecento 1984, p. 17.

279 Mariani 1900, pp. 5-10.

280 Il che è storicamente insostenibile; il problema era risolto esattamente già dal Robolini 1836, V, 2, p. 28.

281 Su di lui Fraccaro 1955, pp. 15-16; per lo sfondo politico delle celebrazioni, vd. Signori 2002, pp. 280-283; per la sua storiografia, in particolare all'interpretazione del Risorgimento, Genovesi 2009, pp. 35-59.

282 Solmi 1920a; Id. 1932. 
Capitolare, Lotario fondava una scuola «chiamandovi i docenti più famosi» - mentre sappiamo che vi era nominato il solo Dúngal - si percepisce che il Solmi sintetizzava e si sbarazzava d'un colpo delle discussioni che, almeno dal Muratori, avevano messo in rilievo la discontinuità tra scuole grammaticali-letterarie affidate a un maestro e le posteriori Universitates d'età comunale.

La pagina successiva del discorso rettorale lascia intravvedere la trama. Il nuovo ordinamento degli studi superiori disciplinato dal Regio Decreto 30 settembre 1923 n. 2102 - cioè la riforma promossa da Giovanni Gentile ministro dell'Istruzione del governo presieduto da Mussolini - prometteva varie provvidenze per le Università, in particolare un'autonomia amministrativa e didattica, ma «a completo carico dello Stato»; esso tuttavia autorizzava la fondazione a Milano di una Università, in concorso fra lo Stato e altri enti pubblici e privati. ${ }^{283}$ Considerando che a Milano dal 1921 era già aperta l'Università Cattolica del Sacro Cuore, il rettore Solmi, mentre elogiava il nuovo ordinamento, sentiva il dovere di «mettere in luce anche i danni» che aveva recato a Pavia, togliendole «il privilegio, conservato per secoli, di essere l'unico centro completo di cultura superiore per la regione lombarda». Quel che restava ora di quel privilegio - in forza d'un complicato accordo che il Solmi spiegava al suo uditorio - era un esiguo protezionismo: ciascuna delle due Università statali, la pavese e la nuova milanese, non avrebbe potuto accettare immatricolazioni di studenti lombardi (fuorché dalla propria provincia) al di sopra d'un certo numero concordato, finché l'altra non avesse raggiunto la stessa soglia di iscritti. Solmi era stato fra le personalità più impegnate nella trattativa, anche come consigliere comunale di Milano: le «convenzioni» che ne erano scaturite realizzavano, se non la ventilata fusione dei due Atenei - quello pavese e il nascituro - in un unico Ateneo lombardo, almeno una rispettiva specializzazione che li coordinasse. Una soluzione che pareva ad alcuni un cedimento, proprio mentre Pavia si rivestiva della «rude armatura» di re Lotario I.

Ma perché proprio Lotario? Nella secolare discussione sull'origo, che abbiamo fin qui seguito, il Capitolare emesso a Corte Olona nell'825 dal nipote di Carlo Magno aveva svolto un ruolo marginale; il

283 Twardzik 2007, pp. 45-63. Il conflitto con Milano risale già alla legge Casati: vd. supra, nt. 256. 
Muratori, che l'aveva pubblicato nel 1725 , vi aveva anzi letto la prova del basso livello culturale dell'Italia medievale; tanto più aveva perso d'importanza da che il Merkel aveva spostato l'attenzione in avanti, sulla scuola giuridica longobardistica sorta intorno al 1000, che distava non solo nel tempo, ma anche nella materia dall'insegnamento grammatical-letterario affidato da Lotario a Dúngal.

Oltretutto, altri candidati per il compito di nobilitare le origini di Pavia non mancavano. Pochi mesi prima, il 25 luglio 1924, Arturo Carlo Jemolo, allora sulla cattedra di Diritto ecclesiastico a Bologna, così commentava in una lettera al collega di disciplina Mario Falco (con il quale l'anno successivo sarebbe stato fra i firmatari del Manifesto di Croce) notizie che davano in fase di stallo la creazione della Facoltà di Giurisprudenza di Milano:

Qui ne giunse la voce, ma credo sia una invenzione dei Bolognesi, per naturale misoneismo solidali con Pavia - purché Pavia non si voglia riannodare a Lanfranco e toccare al primato in vecchiaia di Bologna: se no, son botte! $!^{284}$

Il mot d'esprit accademico s'avvicinava al vero: meno d'un anno più tardi, nel maggio 1925, alla presenza del Re Vittorio Emanuele III insignito della laurea honoris causa, l'Università di Pavia avrebbe inaugurato un monumento a Lanfranco e agli antichi maestri. ${ }^{285}$ Realizzato dallo scultore Gigi Supino, la composizione dava consistenza plastica ai risultati delle ricerche iniziate dal Merkel. ${ }^{286} \mathrm{La}$ statua rappresenta la legge romana che calpesta il delitto; sul basamento sono effigiati i prin-

284 In Jemolo 2005, I, p. 482; la lettera di Jemolo (1891-1981) a Falco (18841943 ) è opportunamente segnalata da di Renzo Villata - Massetto 2007, p. 41. Sulle adesioni pavesi al contromanifesto crociano, vd. Signori 2002, p. 283.

285 La laurea fu deliberata «per acclamazione» il 14 maggio 1925 (ASUPv, Cons. Accademico, verbale, ove è anche riportato il testo latino dell'indirizzo rivolto al Re perché accetti l'onore). Nella stessa seduta si delibera di conferire a tutti i delegati stranieri intervenuti il titolo di Cancellarius.

286 L'idea di un monumento a Lanfranco sembra risalisse a Giacinto Romano: così nel necrologio Solmi 1920b, p. 136, il quale segnala che il Romano «era stato propugnatore di quegli accordi con la metropoli lombarda, che oggi sono in corso di attuazione». Lo scultore Gigi Supino, allievo di Adolfo Wildt, era figlio di Camillo, docente di Economia e poi preside della Facoltà di Giursprudenza. Suo è anche il monumento inaugurato nel 1924 nel cortile della Sapienza di Pisa in memoria degli universitari pisani morti nella Grande Guerra (su cui vd. Caberlin 2010). Esponente di un'importante famiglia di religione israelita, subì più tardi le persecuzioni razziali. 
cipali maestri menzionati nella Expositio ad Librum Papiensem, ossia Lanfranco arcivescovo di Canterbury, Bonfiglio, Bagelardo, Guglielmo e Ugo. ${ }^{287}$ Prende così forma la scuola di Pavia, cui spetta il merito di avere riportato in auge il diritto romano nell'XI secolo, come sottolineò al momento dello scoprimento l'oratore ufficiale, Siro Solazzi. ${ }^{288}$

Ma non era Lanfranco l'eroe eponimo dei Saecularia Undecima, che il Consiglio Accademico aveva deliberato di celebrare tre anni prima, il 12 maggio 1922, nell'imminenza e quasi come risposta al settimo centenario che Padova avrebbe festeggiato di lì a due giorni, il 1417 maggio del 1922, anniversario della migrazione degli studenti bolognesi che avevano dato vita alla nuova Universitas. ${ }^{289} \mathrm{La}$ proposta era stata formulata proprio da Arrigo Solmi, in una lettera all'allora rettore Luigi Berzolari, in cui erano «ricordate le origini dello Studio pavese, che risale al Capitolare di Lotario dell'anno 825, e l'importanza che in detto studio ebbe il celebre giurista Lanfranco». Solmi proponeva quindi «di erigere un monumento e insieme di celebrare nel prossimo anno 1925 l'XI centenario dello Studio pavese, il più antico d'Italia». ${ }^{290} \mathrm{Il}$ Consiglio Accademico aveva approvato con plauso. Dunque, se la figura di Lanfranco richiamava il momento nel quale - secondo quanto accertato dai germanisti - a Pavia era fiorita una riflessione sul diritto

287 Vd. Saecularia Undecima 1925, p. 63, con spiegazione anche dei simboli che richiamano la cristianità.

288 Solazzi (1875-1957), ordinario di Diritto romano, con trascorsi socialisti, costruì il discorso (conservato in ASUPv, Rettorato, carteggio non classificato) su un penetrante esame dell'Expositio, in cui individua «la tendenza a restringere le norme longobarde per dare più largo campo alle leggi romane, ai cui principii con arguta dialettica è tratto il diritto germanico». La conclusione: «A Pavia resta la gloria di aver tenuta accesa la fiamma del nostro diritto, quando ogni altro focolare era spento; e d'avere, prima, e per lunghi duri secoli sola lavorato alla costituzione di un diritto nazionale».

289 L'invito di Padova è menzionato in ASUPv, Cons. Accademico, verbale 24 marzo 1922. Sulla migrazione studentesca a Padova, vd. per tutti Charters 2005, p. 71.

290 ASUPv, Cons. Accademico, verbale 12 maggio 1922. La proposta era già stata avanzata da Luigi Franchi, allora preside di Giurisprudenza, che avrà larga parte nelle pubblicazioni scientifiche del Centenario. Franchi, con Solmi, il rettore Luigi Berzolari e il latinista Carlo Pascal sono nominati membri di una commissione per avanzare «proposte concrete». Vengono aggiunti, nella seduta successiva del 27 maggio, Achille Monti, Giulio Vivanti e Guido Villa «che hanno partecipato come delegati [...] alla cerimonia di Padova». I dubbi di alcuni membri della commissione per «l'inevitabilità di un confronto con la magnifica riuscita della Università di Padova» sono superati, e nella seduta del 24 giugno è deliberato di celebrare l'XI centenario. 
longobardo, capace di trarre le proprie categorie dal diritto romano, nell'XI secolo, il vero punto di riferimento adottato per le celebrazioni fu tuttavia Lotario, di quasi due secoli anteriore. Scelta non senza conseguenze: nonostante l'importanza cruciale riconosciuta a Lanfranco nella storia religiosa e culturale europea, il suo ruolo come giurista è tuttora poco percepito al di fuori della cerchia degli specialisti.

A ben vedere, la scelta di Lotario, anzi del Capitolare dell'825, era solo parzialmente giustificata dalle ricerche che precedettero l'appuntamento, in particolare il «poderoso volume» di Guido Mengozzi (18841960), provetto medievista senese, genero del rettore Solmi di cui aveva sposato la figlia Marianna: non «frutto estemporaneo», ma «risultato di pazienti ricerche», «volle il caso» - osservò un commentatore - «che il libro venisse in luce proprio alla vigilia delle cerimonie universitarie». ${ }^{291}$

Mengozzi riteneva che la scuola di tachigrafia, dal cui tronco s'era diramata quella con indirizzo più giuridico d'età carolingia, fosse stata attiva in «continuità ininterrotta, fino dal tempo romano». ${ }^{292}$ Perciò anche nella sua ricostruzione l'825 non era che un punto lungo una linea: il Capitolare di Lotario riconosceva Pavia «prima scuola del regno» ed era «alla sua volta il riconoscimento dell'attività e dell'importanza che la scuola aveva acquistato prima d'allora». Se si fosse dovuto ricavare dal libro del Mengozzi una data precisa, sarebbe stata piuttosto quella dell'844, in cui lo studioso fissava la creazione della «laurea»:293 una data che - per la celebrazione - aveva tuttavia il difetto d'essere intempestiva. Vero è, ad ogni modo, che il volume del Mengozzi disegnava già un imponente scenario.

291 Bollea 1925, pp. 141-142. Il Mengozzi era figlio di Narciso, storico del Monte dei Paschi; libero docente di Storia del diritto italiano, fu archivista e direttore supplente a Siena, fino alla precoce definitiva dispensa dal servizio nel 1930 per infermità. Sull'ambiente in cui operò, vd. Nardi 2008, pp. 469-480.

292 Mengozzi 1924, p. 34. È cruciale che l'autore contesti la nascita di una scuola giuridica in età ottoniana, come aveva proposto Merkel: fin «dal tempo romano» l'insegnamento pavese aveva avuto carattere giuridico (ivi, pp. 326-328).

293 Mengozzi 1924, pp. 19-25; 321-326: dall'844 la qualifica di iudex et notarius sacri palacii era il «titolo accademico che si conseguiva da coloro che avevano frequentato la scuola di Pavia». Con questo titolo, i migliori accedevano al «tribunale supremo», mentre altri «tornavano al luogo d'origine e ivi esercitavano la funzione di giudice e la professione di notaro» (ed è a quest'ambiente che si deve l'elaborazione su base romanistica di alcuni istituti, caratteristici dei soli placiti del regno, a tutela della proprietà, della libertà e del possesso). Discussa nei particolari, la ricostruzione del Mengozzi rimane «la 
È entro questo scenario che il Capitolare di Lotario assume rilievo nelle pubblicazioni dello stesso Solmi,

le cui ricerche - lo elogiò a festeggiamenti conclusi un collega - furono sempre dirette a mettere in viva luce tutti quegli elementi giuridici e culturali della nostra Storia, onde il Medioevo non appare più come in una vieta e superata concezione, età di tenebre, e ignoranza assoluta, ma bensì come un grande e fecondo periodo di elaborazione di idee e istituti nuovi [...] dimostrazione del formarsi nella capitale dei regni goto, longobardo e franco di un grande focolare di cultura superiore, il più antico del mondo intero. ${ }^{294}$

Per comprendere, e giudicare equanimemente, questo intreccio di scienza e di politica accademica bisogna ricordare che, anche il 1088, quale anno di fondazione dell'Università di Bologna, fu scelto convenzionalmente, ossia in funzione delle celebrazioni. Lo dichiarava lealmente lo stesso «Manifesto» emanato nel febbraio 1887 dal Comitato esecutivo presieduto dal rettore, il geologo Giovanni Cappellini (1833-1922), in cui sedeva il Carducci: «Non il giorno si può fissare né l'anno» della fondazione, riconoscevano gli estensori, dicendosi peraltro certi che la ricorrenza cadesse negli «ultimi lustri» del XIX secolo. Su questa premessa, «La Università dei professori e degli studenti e la città tutta accolsero con plauso la proposta di commemorare le gloriose origini nella primavera del 1888»:295 la data di fondazione fu dunque calcolata a ritroso partendo dalla data di celebrazione, pur senza fare torto alla storia.

Il meccanismo pavese è analogo. Su uno sfondo storico consistente, fu scelto un emblema. Il nipote di Carlo Magno I era figura per la verità abbastanza sbiadita nell'immaginario, ma si prestava per una ragione che non mi pare sia stata ben messa a fuoco: non Lotario, ma il Capitolare di Lotario emanato a Corte Olona nel maggio 825 fu preso a punto di riferimento, ossia un documento normativo con una data precisa ed emanato da un re. Era la retroproiezione del diploma di

plus célèbre et la plus fouillé»: vd. la fondamentale indagine di Bougard 1995, spec. pp. 130-133; 307-339; l'A. riconosce che furono i giudici di Pavia i proseliti delle nuove procedure, e incrementarono il proprio ruolo nel periodo "nazionale", fra la morte di Ludovico II nell'875 e Ottone I; quanto ai placiti, pone intorno all'810 una modifica nel formulario dei notai pavesi); cfr. Padoa Schioppa 2012, pp. 146; 162.

294 Così l'ordinario di Filosofia Guido Villa (1867-1949), in ASUPV, Cons. Accademico, verbale 7 ottobre 1925. Lo scritto più emblematico è Solmi 1925.

295 Carducci 1888, pp. 7-9. 
Carlo IV. Un uso analogo ne era stato già fatto a metà del Settecento se n'è accennato - quando il Capitolare fu allegato a una supplica dei pavesi a Maria Teresa, per dimostrare il diritto della città a essere sede della scuola, che anche allora si temeva fosse spostata a Milano. ${ }^{296} \mathrm{Il}$ documento, che per il Muratori dimostrava invece la rovina del panorama culturale all'altezza del IX secolo, riprendeva così la sua validità in chiave giuridica.

Che sia stato uno storico del diritto come Solmi a favorire questa scelta, era consono alla sua prospettiva culturale. Naturalmente, che il Capitolare coincidesse anche con il momento in cui Pavia doveva rispondere a Padova e celebrare la sua maggiore antichità rispetto a Milano - alla presenza del re d'Italia - era un ottimo argomento a favore. Un delegato dell'Università di Neuchâtel, autore di un vivace resoconto delle celebrazioni, ne colse senza schermi lo spirito:

Cette petite lumière, allumée en 825 , deviendra plus tard un grand flambeau de science, qui rayonnera sur toute l'Italie. Elle a été la première à briller après Rome, il était bon de le rappeller, au moment où s'érigeaient en face, à l'américaine, des établissements sans passé et sans tradition, qui s'imaginaient que pour faire de la science il n'y avait besoin que d'argent. ${ }^{297}$

Le celebrazioni durarono tre giorni e furono un «magnifico successo» ${ }^{298}$

A leggere dal palco allestito nel Castello Visconteo il discorso in rappresentanza del presidente del Consiglio fu Pietro Fedele (18731943), dal gennaio di quell'anno ministro dell'Istruzione Pubblica subentrato ad Alessandro Casati che si era dimesso per protesta contro il discorso di Mussolini alla Camera del 3 gennaio sul delitto Matteotti. ${ }^{299}$ Sul piano storico, si sente che il Fedele - a sua volta note-

296 Vd. supra, nt. 216, a proposito della supplica redatta da Francesco Caimi.

297 Junod 1925, p. 5.

298 Così l'ordine del giorno del Senato accademico del 16 giugno 1925: ASUPv, Cons. Accademico, verbale. Documentazione della preparazione e svolgimento dei Saecularia Undecima in ASUPv, Rettorato, Carteggio non schedato e nella corrispondenza di Plinio Fraccaro, che fu commissario delle celebrazioni: BUPv, Ticinesi, 819, B 27 (Carte Plinio Fraccaro Varie 21). Oltre al menzionato resoconto di Junod (non immune da concezioni sociali e culturali che recano i segni del tempo), vd. Malcovati 1985, pp. 243-254.

299 Biscione 1995, pp. 572-575. 
vole medievista - aveva ben presenti le pagine di Mengozzi e di Solmi; sul piano politico, rovescia lo schema di Merkel. ${ }^{300}$ Pavia è vista come custode delle «tradizioni classiche» di fronte all'urto degli invasori: «Non si tratta quindi di un'istituzione straniera che sia stata trapiantata fra noi dal franco Lotario; ma di una scuola italiana, che, per necessità pratiche, ha coltivato ora questo ora quel diritto barbarico, ma non ha mai abbandonato il glorioso diritto nostro». ${ }^{301}$ Soprattutto, è rivelatrice l'interpretazione che offre del Capitolare di Lotario: «[...] non fa che disciplinare la libertà della scuola, assegnandole limiti e fini ben determinati, trasformandola in una vera e propria scuola di Stato, che dipende direttamente dal Sovrano». Si vede bene che, in quanto norma, il Capitolare si prestava efficacemente a essere inteso come atto fondativo, anzi a essere accostato alla riforma Gentile, di cui il Fedele doveva essere l'attuatore; anzi, ancor più specificamente, poteva ben connettersi al problema della concorrenza fra Pavia e Milano, cui infatti il ministro allude nella chiusura dell'orazione: «ho la certezza che l'Università di Pavia ha ancora una propria missione da compiere [...]. La riforma, attuata dal Governo Nazionale, assicura la libertà alle Università ciascuna delle quali potrà svolgere un proprio compito particolare».

Il nipote si sostituì dunque a Carlo Magno, oscurando e facendo addirittura svanire la memoria dell'avo come fondatore della scuola di Pavia, mito che s'era invece imposto in Europa dal Cinquecento per circa tre secoli, forte anche del sincronismo con Parigi, ma che aveva il difetto di appoggiarsi su un racconto vago, privo di data, e su un personaggio senza volto legato a un'ambientazione ecclesiastica, il monaco mercante di sapienza inviato al monastero di Sant'Agostino.

300 Il suo discorso può essere incluso fra gli esempi della circolazione dei temi medievistici nel discorso pubblico in età fascista, su cui richiama l'attenzione Moretti 2007 , pp. 155-174, che accenna al ruolo svolto proprio da Fedele nelle celebrazioni del settimo centenario della morte di san Francesco d'Assisi nel 1926, ove fu protagonista del «famoso avvenimento politico conosciuto col nome di incontro di Assisi», con il cardinale Raffaele Merry del Val, che prefigurava il riavvicinamento concordatario; vd. Irace 2003, pp. 209-226.

$301 \ll$ La cultura non solo non perisce, ma trionfa sull'ignoranza e sulla violenza dei dominatori e riveste di lingua latina il pensiero germanico»: con la chiosa «e romanità per noi è nazionalità». Il discorso è conservato, dattiloscritto su velina, in ASUPV, Rettorato, carte non classificate, da cui tutte le citazioni. 
La costruzione del nuovo racconto di fondazione fu rapida: Lotario I fu effigiato nello stendardo (intreccio anch'esso di parti antiche e nuove) che a nome delle «signore pavesi» Lina Golgi, moglie del Nobel, consegnò al rettore Solmi il 20 maggio, all'apertura dei festeggiamenti, e la data dell'825 è ribadita nella formula diventata canonica nelle inaugurazioni dell'anno accademico. ${ }^{302}$

Questa costruzione si riverbera sul piano storiografico. Il peso strumentalmente attribuito al Capitolare di Lotario nel 1925 ha creato un alone di diffidenza intorno alla questione delle origini, come se evocare il quadro culturale anteriore al 1361 significasse arretrare alle dispute municipalistiche pre-muratoriane; forse così si spiegano le tante prudenze nel riconoscere il collegamento fra Pavia e l'indubbio emergere, intorno al 1000, di una raffinata scuola giuridica capace di recuperare il diritto romano in funzione costruttiva rispetto al diritto longobardo. ${ }^{303}$ È fuori di dubbio che le fondazione viscontea sia stata l'atto iniziale di una nuova istituzione. Ma l'avere ridotto la questione pavese a quella dello Studium ha fatto perdere di vista - e tuttora rischia di non fare vedere fino in fondo - la straordinaria impresa intellettuale che è stata qui compiuta da Lanfranco e dagli altri giuristi nell'arco di tre o quattro generazioni.

Siamo al termine del tragitto, che ci ha condotto dal XIV al XX secolo. Se è vero che ogni ragionamento del passato muove da interessi attuali, ciò vale specialmente per la storia universitaria, proprio perché chi la narra vi è quasi sempre immerso in prima persona; la vita stessa dell'Università - gli anniversari, le crisi, i momenti di riforma, le fasi di vigore - si ripercuote quasi senza mediazioni su chi si curva sul suo passato. Avere individuato - per così dire dall'interno - alcuni schemi argomentativi, il farsi e il disfarsi di risposte, e insieme avere collegato la riflessione sul passato alle vicende dell'Università non può garantire l'immunità da condizionamenti, ma aiuta a percepire la profonda storicità dell'istituzione e di qualunque pensiero che si possa formulare su di essa.

302 Sul gonfalone, vd. approfonditamente Erba 2008a.

303 È merito di Radding 1988 avere spostato l'attenzione dal terreno scolastico al versante intellettuale; vd. anche Radding - Ciaralli 2007, pp. 73-99. Questa interpretazione è discussa: bibliografia in Melve 2007, p. 354, nt. 27; positivamente Bougard 1995; Padovani 2007, pp. 82-85; cfr. Padoa Schioppa 2012, pp. 143-164. 


\section{RIFERIMENTI BIBLIOGRAFICI}

M. Alciato 1600, Responsum seu Tractatus de praecedentia inter feudatarium Caesarei, Pontificiique iuris doctorem, et feudatarium babentem annexam comitatus et marchiae dignitatem, Ticini, In aedibus Andreae Viani, 1600.

Alessandro da Rho 1587, Tractatus de analogis, univocis et aequivocis iuris utriusque Decisiones amplissimi Senatus Mediolanensis et aliorum supremorum magistratuum includens, Venetiis, Ex officina Ioannis Baptistae Somaschi, 1587.

Alessandro da Rho 1592, Oratio [...] de praestantia civitatis gymnasiique Ticinensis ad illustriss. et excell. Heroem Laurentium Suarez de Figueroa, et Corduba Ducem de Feria, Habita Ticini die xviii Februarii mdxcii, Ticini, Apud Haeredes Hieronymi Bartoli, 1592.

Alessandro da Rho 1595, Consiliorum sive Responsorum et Decisionum liber primus, Venetiis, Ex officina Iacopi Antonii Somaschi, 1595.

Almum Studium Papiense 1,I = Almum Studium Papiense. Storia dell'Università di Pavia, Volume 1. Dalle origini all' età spagnola, Tomo I. Origini e fondazione dello Studium generale, a c. di D. Mantovani, Milano, Cisalpino Istituto Editoriale Universitario, 2012.

Almum Studium Papiense. Storia dell'Università di Pavia, 1,II = Almum Studium Papiense. Storia dell'Università di Pavia, Volume 1. Dalle origini all'età spagnola, Tomo II. L'età spagnola, a c. di D. Mantovani Milano, Cisalpino Istituto Editoriale Universitario, 2013.

N. Álvarez de las Asturias 2008, La "Collectio Lanfranci". Origine e influenza di una collezione della Chiesa anglo-normanna, Milano, Giuffrè, 2008.

Antonino Fiorentino, santo 1527, Secunda pars Historiarum domini Antonini archipresulis Florentini in tribus tomi discretarum, Lugduni, Industria Jacobi Myt, 1527.

F. Arese Lucini 2008, Carriere, magistrature e stato. Le ricerche di Franco Arese Lucini per l'Archivio Storico Lombardo (1950-1981), a c. di C. Cremonini, Milano, Cisalpino, 2008.

L. Ariosto 1974, Tutte le opere, IV. Le commedie, a c. di A. Casella - G. Ronchi - E. Varasi, Milano, Mondadori, 1974.

E. Artifoni 2007, Le questioni longobarde. Osservazioni su alcuni testi del primo Ottocento storiografico italiano, Mélanges de l'École Française de Rome. Moyen Âge, 119 (2007), 297-304.

L. Avellini 1990, a c. di, Sapere elo potere. Discipline, dispute e professioni nell'Università medievale e moderna: il caso bolognese a confronto. Atti del IV Convegno (Bologna, 13-15 aprile 1989), I. Forme e oggetti della disputa delle arti, Bologna, Istituto per la storia di Bologna, 1990.

P. Azario 1730, Chronicon de gestis principum Vicecomitum ab anno mccl usque ad annum mcclxii, a c. di L.A. Muratori, Rerum Italicarum Scriptores ab anno aerae Christianae quingentesimo ad millesimumquingentesimum, XVI, Mediolani, Ex typographia Societatis Palatinae, 1730.

P. Azario 1927, Liber Gestorum in Lombardia, a c. di F. Cognasso, Rerum Italicarum Scriptores. Raccolta degli storici italiani dal Cinquecento al Millecinquecento, XVI.4, Bologna, Zanichelli, 1927. 
E. Barbieri 2011, I contenuti del primo privilegio, in Mantovani - Valeriani 2011, 24-26.

M.M. Bassi Costa 1951, Le origini dello studio di Pavia. Formazione della scuola di Pavia nell'alto Medioevo, Annali della Biblioteca Governativa e Libreria Civica di Cremona 4 (1951), 9-48.

M. Bellabarba 1999, Honor Discipline and the State. Nobility and Justice in Northern Italy (Fifteenth to Seventeenth Centuries), in H. Schilling, a c. di, Institutionen, Instrumente und Akteure sozialer Kontrolle und Disziplinierung im frübneuzeitlichen Europa, Frankfurt am Main, Klostermann, 1999, 225-250.

A. Belloni 1982, Giovanni Dondi, Albertino da Salso e le origini dello Studio pavese, Boll. Soc. Pavese St. Patria 82 (1982), 17-49.

A. Belloni 1985, Signorolo Omodei e gli inizi della scuola giuridica pavese, Boll. Soc. Pavese St. Patria 85 (1985), 29-39.

S. Benz 2002, Jakob Middendorp und die Anfänge der Wissenschaftsgeschichte in Köln, Geschichte in Köln 49 (2002), 105-130.

D. Bianchi 1964, Giovanni Gaspare Beretti, Boll. Soc. Pavese St. Patria 64 (1964), 1-34.

P.G. Bietenholz 1994, Historia and Fabula. Myths and Legends in Historical Thought from Antiquity to the Modern Age, Leiden - New York - Köln, Brill, 1994.

F.M. Biscione 1995, Fedele, Pietro, DBI 45 (1995), 572-575.

R. Bizzocchi 2009, Genealogie incredibili. Scritti di storia nell'Europa moderna, Bologna, il Mulino, 2009.

L.C. Bollea 1925, Le pubblicazioni dell'undecimo centenario dell'Università di Pavia (II), Boll. Soc. Pavese St. Patria 25 (1925), 141-167.

A. Boretius 1864, Die Capitularien im Langobardenreich. Eine rechtsgeschichtliche Abhandlung, Halle, Buchhandlung des Waisenhauses, 1864.

L. Bossi 1822, Notizie compendiose della vita e degli scritti di Siro Comi, Milano, Tipografia di Commercio, 1822.

F. Bougard 1995, La justice dans le Royaume d'Italie de la fin du VIII siècle au début du XI e siècle, Rome, École Française de Rome, 1995.

M. Brennan 1986, Materials for the Biography of Johannes Scottus Eriugena, Studi Medievali 27 (1986), 413-460.

H. Bresslau 1921, Geschichte der Monumenta Germaniae historica, Neues Archiv der Gesellschaft für ältere deutsche Geschichtskunde 42 (1921), 1-769.

S. Breventano 1570, Istoria della antichità, nobiltà et delle cose notabili della città di Pavia, In Pavia, Appresso Hieronimo Bartoli, 1570.

C. Brühl - C. Violante 1983, Die "Honorantie civitatis Papie". Transkription, Edition, Kommentar, Köln - Wien, Böhlau, 1983.

J.A. Brundage 2008, Medieval Origins of the Legal Profession. Canonists, Civilians, and Courts, Chicago, University of Chicago Press, 2008.

E. Bufacchi 2006, Maggi, Carlo Maria, DBI 68 (2006), 328-232.

D.A. Bullough 1964, Le scuole cattedrali e la cultura dell'Italia settentrionale prima dei Comuni, in Vescovi e diocesi in Italia nel Medioevo (sec. IX-XIII). Atti del II Convegno di storia della Chiesa in Italia (Roma, 5-9 settembre 1961), Padova, Antenore, 1964, 111-143 (ried. in R. Greci 1996, a c. di, Il pragmatismo degli intellettuali. Origini e primi sviluppi dell'istituzione universitaria, Torino, Scriptorium, 1996 23-46). 
F. Caberlin 2010, Legittimazione della Grande Guerra e culto dei caduti: il caso delle Università toscane, Quaderni su guerre e memoria del '900 - Istrevi (2010), on line.

P. Cabrini 1976, Capsoni, Siro Severino, DBI 19 (1976), 242-244.

P. Cabrini 1982, Comi, Siro, DBI 27 (1982), 565-567.

M. Càmpori 1901, a c. di, L.A. Muratori, Epistolario, II. (1699-1705), Modena, Società Tipografica Modenese, 1901.

H. Canisius 1601, Antiquae Lectionis Tomus I, in quo XVI antiqua monumenta ad Historiam Mediae aetatis illustrandam nunquam edita, Ingolstadii, Ex officina typographica Edereiana, Apud Andream Angermarium, 1601.

S.S. Capsoni 1769, Origini e privilegi della Chiesa Pavese aggiuntavi la serie cronologica de' vescovi di essa. Ragionamento apologetico, Pavia, Bolzani, [1769].

S.S. Capsoni 1782-1788, Memorie istoriche della Regia Città di Pavia e suo territorio antico e moderno, I-III, Pavia, Stamperia del R.I. Monistero di San Salvatore, 1782-1788.

A. Caracciolo 1968, Domenico Passionei, tra Roma e la repubblica delle lettere, Roma, Edizioni di Storia e Letteratura, 1968.

G. Carducci 1888/1968, Lo Studio bolognese. Discorso di Giosuè Carducci per l'Ottavo Centenario, Bologna, Zanichelli, 1888 (ried. a c. di P. Fiorelli, Firenze, Università degli Studi, 1968).

V. Castronovo 1971, Bossi, Gerolamo, DBI 13 (1971), 305-307.

E. Cau - M.A. Casagrande Mazzoli 1987, Cultura e scrittura a Pavia (secoli V-X), in Storia di Pavia, II, 177-217.

A.G. Cavagna 1980, Antonio Maria Spelta, storico e letterato a Pavia tra Cinque e Seicento, Annali di Storia Pavese 2-3 (1980), 251-263.

A.G. Cavagna 1981, Libri e tipografi a Pavia nel Cinquecento. Note per la storia dell'Università e della cultura, Milano, Cisalpino - Goliardica, 1981.

M. Cavina 2003, Il duello giudiziario per punto d'onore. Genesi, apogeo e crisi nell'elaborazione dottrinale italiana (sec. XIV-XVI), Torino, Giappichelli, 2003.

A. Cerri 1992, Francesco Petrarca a Pavia, in Storia di Pavia, III.1, 451-495.

Charters $=$ Charters of Foundation and Early Documents, Leuwen, Leuven University Press, 2005.

B. Chasseneuz, de 1529, I-XII, Catalogus Gloriae mundi, Laudes, honores, excellentias ac praeeminentias omnium fere statuum, plurimarumque rerum illius continens, Lugduni, Per Dionysium de Harsy, 1529.

A. Cipollini 1900, Carlo Maria Maggi sopraintendente all'Università di Pavia, Archivio Storico Lombardo 27 (1900), 305-318.

Clarorum Venetorum ad Ant. Magliabechium nonnullosque alios Epistolae 1745, I, Florentiae, Ex Typographia ad Insigne Apollinis in Platea Magni Ducis, 1745.

C.J. Classen 1980, Die Stadt im Spiegel der Descriptiones und Laudes urbium in der antiken und mittelalterlichen Literatur bis zum Ende des zwölften Jahrbunderts, Hildesheim - New York, G. Olms, 1980.

$\mathrm{Ph}$. Clüver 1697, Introductio in universam geographiam tam veterem quam novam, tabulis geographicis XLVI ac notis olim ornata a Johanne Bunone, jam vero locupleta- 
ta additamentis \& annotationibus Joh. Frid. Hekelii \& Joh. Reiskii, Amstelaedami, Apud Joannem Wolters, 1697.

E.W. Cochrane 1981, Historians and Historiography in the Italian Renaissance, Chicago - London, The University of Chicago Press, 1981.

Codice diplomatico, $\mathrm{I}=$ Codice diplomatico dell'Università di Pavia raccolto ed ordinato dal sac. dott. Rodolfo Maiocchi, I. 1361-1400, Pavia, Tipografia successori Fratelli Fusi, 1905 (rist. 1971).

Codice diplomatico, II.1 = Codice diplomatico dell'Università di Pavia, II.1. 1401-1440, Pavia, Tipografia successori Fratelli Fusi, 1913 (rist. 1971).

Codice diplomatico, II.2 = Codice diplomatico dell'Università di Pavia, II.2. 1441-1450, Pavia, Tipografia successori Fratelli Fusi, 1915 (rist. 1971).

F. Collard 1996, Un Historien au travail à la fin du XVe siècle: Robert Gaguin, Genève, Droz, 1996.

A. Collins 2007, Teacher in Faith and Virtue. Lanfranc of Bec's Commentary on Saint Paul, Leiden - Boston, Brill, 2007.

G. Coluccia 2001, L'esperienza teatrale di Ludovico Ariosto, Lecce, Manni, 2001.

S. Comi 1783, Franciscus Philelphus Archigymnasio Ticinensi Vindicatus a Syro Comi. Plura intercessere de re scholastica eiusdem urbis ante Galeatium II. Vicecomitem, Ticini, in Typ. Monast. S. Salvatoris, 1783.

E. Conte 2009, Diritto comune. Storia e storiografia di un sistema dinamico, Bologna, il Mulino, 2009.

Contributi alla storia dell'Università di Pavia $=$ Contributi alla storia dell'Università di Pavia pubblicati nell'XI centenario dell'Ateneo, Pavia, Tipografia Cooperativa, 1925.

A. Corbellini 1915, Appunti sull'Umanesimo in Lombardia, Boll. Soc. Pavese St. Patria 15 (1915), 327-362.

B. Corio 1978, Storia di Milano, I-II, a c. di A. Morisi Guerra, Torino, UTET, 1978.

A. Cornazzano 1643, Oratio babita in Papiensi Academia prid. Id. Octob. 1553 ab olim Aldigerio Cornazzano Papiensi patricio [...] in adeptione Iurisprudentiae Laureae clarissimo saeculi illius I.C. Thoma Gualla Papiensi laureatore, Papiae, Ex Typographia Io. Andreae Magri, 1643.

M. Cortesi, in M. Azzolini - M. Cortesi - C. Crisciani - M. Nicoud - P. Rosso, La Facoltà di Arti e Medicina, in Almum Studium Papiense 1,I, 515-570.

F. Corti, iun. 1533, Praeclara commentaria in primam et secundam Codicis partes, Lugduni, Joannes Moylin excudebat, 1533.

G.B. Costa 1610, Tractatus [...] de portione rata, Venetiis, Apud Societatem Minimam, 1610.

A. Cottignoli 1984, a c. di, Edizione Nazionale del Carteggio di L. A. Muratori, XXII. Carteggio con Giovan Gioseffo Orsi, Firenze, Olschki, 1984.

R. Crotti 2012, L'istituzione dello Studium generale, in Almum Studium Papiense 1,I, 237-280

R. Crotti - P. Majocchi 2005, a c. di, La rubrica degli atti di Albertolo Griffi: notaio e cancelliere episcopale di Pavia (1372-1420), Milano, Unicopli, 2005.

J.A. Crusius 1665, Tractatus politico-juridico-bistoricus de praeeminentia, sessione, prae- 
cedentia, et universo jure proedrias Magnatum in Europa, Bremae, Impensis \& typis Jacobi Kohleri, 1665.

G. D'Onofrio 1993, a c. di, Lanfranco di Pavia e l'Europa del secolo XI. Nel IX centenario della morte, 1089-1989. Atti del Convegno internazionale di studi (Pavia, Almo Collegio Borromeo, 21-24 settembre 1989), Roma, Herder, 1993.

G. D'Onofrio 2009, I fondatori di Parigi. Giovanni Scoto e la teologia del suo tempo, in I. Biffi - C. Marabelli, a c. di, Figure del pensiero medievale, I. Fondamenti e inizi. IV-IX secolo, Milano - Roma, Jaca Book - Città Nuova, 2009, 435-470.

F.A. Dal Pino 1975, Capassi, Gerardo, DBI 18 (1975), 387-391.

S. Dale 2007, Fourteenth-Century Lombard Chronicles, in S. Dale - A. Williams Lewin - D.J. Osheim, a c. di, Chronicling History. Chroniclers and Historians in Medieval and Renaissance Italy, University Park, The Pennsylvania State University, 2007, 170-195.

M. De Bernardi 1936, Un secolo di erudizione pavese 1750-1850, Boll. Soc. Pavese St. Patria 36 (1936), 7-72.

G. De Gioannis Gianquinto 1864, Diritto amministrativo d'Italia informato al carattere nazionale, Pavia, Bizzoni, 1864.

C.A. De Luca 1689, De praestantia laureae doctoralis iuristarum, Neapoli, Ex typographia Francisci Mollo, 1689.

Decreta et Rescripta 1618 = Decreta et Rescripta excelleniss. [sic] Gubernatorum ac ampliss. Senatus Mediolani, de administratione, et regimine Academiae Ticinensis, directa ad perillustres eiusdem civitatis Praetores. Quibus accesserunt, et Edicta in hoc genere ab ipsis Praetoribus condita, Ticini, Apud Iacobum Ardizzonum, 1618.

DBI = Dizionario Biografico degli Italiani, Roma, Istituto della Enciclopedia Italiana, 1960-.

E. Dezza 1992, Gli statuti di Pavia, in Storia di Pavia, III.1, 409-431.

E. Dezza 2008, Antonio Gatti e la riscoperta della dimensione umanistica della giurisprudenza all'inizio del secolo dei lumi, in Tra diritto e storia. Studi in onore di Luigi Berlinguer promossi dalle Università di Siena e di Sassari, I, Soveria Mannelli, Rubbettino, 2008, 791-814.

P. Di Pietro Lombardi 1996, Girolamo Tiraboschi, Rimini, Luisè, 1996.

M.G. di Renzo Villata - G. Massetto 2007, La "seconda" Facoltà giuridica lombarda. Dall'avvio agli anni Settanta del Novecento, Annali di Storia delle Università Italiane 11 (2007), 41-78.

M.G. di Renzo Villata - G. Massetto 2012, La Facoltà legale. L'insegnamento del Diritto civile (1361-1535), in Almum Studium Papiense, 1,I, 467-484.

C. Dionisotti 1971, Filologia umanistica e testi giuridici fra Quattro e Cinquecento, in La critica del testo. Atti del secondo Congresso internazionale della Società Italiana di Storia del Diritto, Firenze, Olschki, 1971, 189-204.

C. Dossi 2010, Note azzurre, a c. di D. Isella, Milano, Adelphi, 2010.

C.E. Du Boulay 1665, Historia Universitatis Parisiensis, ipsius fundationem, nationes, facultates, magistratus, decreta [...] a Carolo M. ad nostra tempora ordine chronologico complectens, I. Ab anno circiter 800. ad annum 1100., Parisiis, Apud Franciscum Noel, 1665.

C.E. Du Boulay 1670, Historia Universitatis Parisiensis, plurimorum collegiorum fundationes, statuta, privilegia reformationes [...] complectens, V. Ab anno 1400. ad 
annum 1500, Parisiis, Apud Petrum de Bresche et Iacobum de Laize de Bresche, 1670.

H.H. Egglmaier 1997, Graf Thun und das Rechtsstudium an den oberitalienischen Universitäten Padua und Pavia. Ein Beitrag zur Bildungs- und Wissenschaftspolitik im Alpen-Adria-Raum, in Forschungen zur Geschichte des Alpen-Adria-Raumes. Festgabe für Othmar Pickl zum 70. Geburtstag, Graz, Instituts für Geschichte der Karl-Franzens - Universität Graz, 1997, 107-118.

L. Erba 2008a, I palazzi del sapere a Pavia dal XIV al XVIII secolo. L'Università, $i$ Collegi, l'Orto botanico, in B. Azzaro, a c. di, L'Università di Roma "La Sapienza" e le università italiane, Roma, Gangemi, 2008, 147-158.

L. Erba 2008b, Il gonfalone dell'Università di Pavia, Boll. Soc. Pavese St. Patria 108 (2008), 115-144

L. Erba 2011, La collezione di lapidi dell'Università di Pavia, in Mazzilli Savini 2011, 57 81.

A. Esch 2006, Elementi di una nuova dinamica: le origini dell'università e il nuovo interesse per l'antico, in A. Barbero, a c. di, Storia d'Europa e del Mediterraneo, II. Dal medioevo all'età della globalizzazione, 4. Il Medioevo (secoli V-XV), VIII. Popoli, poteri, dinamiche, Roma, Salerno, 2006, 709-740.

A. Fabbroni 1781, Vitae Italorum doctrina excellentium qui saeculis XVII et XVIII floruerunt, VII, Pisis, Excudebat Jacobus Gratiolus, 1781.

E. Farnese 1594, De simulacro Reipublicae sive De imaginibus politicae et oeconomicae virtutis, Papiae, Ex officina typographyca Andreae Viani, 1594.

G. Fasoli 1974, Carlo Magno nelle tradizioni storico-leggendarie italiane, in Ead., Scritti di storia medievale, a c. di F. Bocchi - A. Carile - A.I. Pini, Bologna, Fotocromo Emiliana, 891-916.

E. Ferraglio 2010, Frammenti ticinesi del carteggio tra Lodovico Antonio Muratori e Antonio Gatti, Bollettino Storico della Svizzera Italiana 113 (2010), 151-168.

A. Ferraresi 1986, La miscellanea Belcredi, in A. Ferraresi - A. Mosconi Grassano - A. Pasi Testa, Cultura e vita universitaria nelle miscellanee Belcredi, Giardini, Ticinensia, Milano, Cisalpino - Goliardica, 1986, 17-208.

G. Fiamma 1869, Chronicon maius, in A. Ceruti, a c. di, Chronicon extravagans et Chronicon maius auctore Galvano Flamma (Miscellanea Storica Italiana, 7, 1869), 506-784.

S. Fiaschi 2012, La cattedra, la corte e l'archivio. Umanesimo e produzione storiografica tra Milano e Pavia nel '400, in Almum Studium Papiense 1,I, 743-760.

P. Fraccaro 1955, Parole del Magnifico Rettore dell'università di Pavia, in Onoranze ai fratelli Edmondo e Arrigo Solmi, Pavia, Tip. C. Re, 1955, 15.

L. Franchi 1925, Giacomo Parodi. Cenno, in Contributi alla storia dell'Università di Pavia, 355-362.

C. Frova 2012, La nascita delle Università nella storia del Medioevo italiano, in Almum Studium Papiense 1,I, 13-28.

E. Fugazza 2012a, Il diploma di Carlo IV, in Almum Studium Papiense 1,I, pp. 229-232.

E. Fugazza 2012b, Il trasferimento dello Studium a Piacenza (1398-1402), in Almum Studium Papiense 1,I, 325-330. 
M. Füssel 2011, The Conflict of the Faculties: Hierarchies, Values and Social Practices in Early Modern German Universities, History of Universities 25 (2011), 80110.

E. Gabba 2000, Pavia. Domicilium sapientie. Note storiche, Como, New Press, 2000.

E. Gabba - D. Magnino 1994, Angelo Teodoro Villa e l'insegnamento della storia nell'Università di Pavia nel Settecento, RIL, cl. sc. mor. st. 128 (1994), 297-315.

M. Gabriele 2011, An Empire of Memory. The Legend of Charlemagne, the Franks, and Jerusalem before the First Crusade, Oxford, Oxford University Press, 2011.

R. Gaguin 1504, Compendium [...] super Francorum gestis ab ipso recognitum \& auctum, Parisiis, impensis Ioannis Parvi, 1504.

A.E. Galeotti 1978, Politica della cultura e istituzioni educative. La riforma dell'Università di Pavia (1753-1790), Pavia, Centro Studi sull'Illuminismo lombardo, 1978.

E. Garin 1947, a c. di, La disputa delle arti nel Quattrocento. Testi editi ed inediti, Firenze, Vallecchi, 1947.

E. Garin 1969, Leggi, diritto e storia nelle discussioni dei secoli XV e XVI, in Id., L'Età nuova. Ricerche di storia della cultura dal XII al XVI secolo, Napoli, Morano, 1969, 237-260.

F. Gasti 2012, Cultura e scuola a Pavia nell'età di Ennodio e Boezio, in Almum Studium Papiense 1,I, 105-114.

A. Gatti 1703, Diatriba de iure literae et dignitate inter materiam civilem et criminalem, authore I.C. Antonio Gatto Derthonense, publico I.C. Professore in Ticinensi Universitate, Ticini, s.e., 1703.

A. Gatti 1704, Gymnasii Ticinensis historia, et vindiciae a saeculo V ad finem XV et plura de ejusdem urbis antiqua nobilitate, Mediolani, Typis Iosephi Pandulfi Malatestae, 1704.

A. Gatti 1709a, a c. di, Nugae Laderchianae in Epistola ad Equitem Florentinum sub nomine, et sine nomine Donati Polidori vulgata. Centuria prima accurante $M$. Antonio Gatto, Genuae, Typ. Io. Mariae Ferroni, 1709.

A. Gatti 1709b, De recte instituenda Iuris Academia, ad Lamindum Pritanium nobilissimum, et eruditissimum virum, Epistula, Venetiis, s.e., 1709.

[A. Gatti] 1710, Articolo IX, in Giornale de' Letterati d'Italia, IV, Venezia, Gio. Gabriello Ertz, 1710, 225-252.

A. Gatti 1722, Historia Gymnasii Ticinensis a saeculo V ad finem saeculi XV nec non de eius urbis antiqua nobilitate. Editio novissima, in J.G. Graevius, a c. di, Thesaurus antiquitatum et historiarum Italiae. Cum praefationibus Petri Burmanni, IV.2. Lugduni Batavorum, excudit Petrus Vander, 1722.

S. Gavinelli 2012, Dúngal e l'organizzazione scolastica del Regnum Italicum in età carolingia, in Almum Studium Papiense 1,I, 115-128

P. Genovesi 2009, Il manuale di storia in Italia. Dal fascismo alla Repubblica, Milano, FrancoAngeli, 2009

M. Gibson 1989, Lanfranco. Da Pavia al Bec a Canterbury, Milano, Jaca Book, 1989.

Giovanni da Imola 1525, Super Clementinis [...] commentaria, Lugduni, Vincentius de Portonariis, 1525. 
P. Giovio 1549, Le vite dei dodici Visconti prencipi di Milano, tradotte per $m$. Lodovico Domenichi, In Vinegia, Appresso Gabriel Giolito, 1549.

P. Giovio 1552, Le iscrittioni poste sotto le vere imagini de gli buomini famosi in lettere [...] tradotte di latino in volgare da Hippolito Orio ferrarese, in Fiorenza, Appresso Lorenzo Torrentino, 1552.

D. Girgensohn 1993, Francesco Zabarella aus Padua. Gelebrsamkeit und politisches Wirken eines Rechtsprofessors während des großen abendländischen Schismas, Zeitschrift der Savigny-Stiftung für Rechtsgeschichte. Kan. Abt. 79 (1993), 232277.

P. Golinelli 2008, Il Codice delle leggi della Cattedrale di Modena. Descrizione, in Leges Salicae 2008, 17-35.

F.A. Goria 2010, Fra rinnovamento e tradizione. Lo "Speculum feudorum" di Claude de Seyssel, Milano, Giuffrè, 2010.

U. Gualazzini 1969, La scuola pavese con particolare riguardo all'insegnamento del diritto, in Pavia capitale di Regno, 35-73.

G. Guderzo 2011, Amore di Pavia, Milano, Unicopli, 2011.

W. Hammer 1940, Balthazar Rasinus and his Praise of Studies at the University of Pavia, Studies in Philology 37 (1940), 133-148.

A. Hortis 1874, Scritti inediti di Francesco Petrarca, Trieste, Tip. del Lloyd austro-ungarico, 1874.

P.F. Howard 1995, Beyond the Written Word. Preaching and Theology in the Florence of Archbishop Antoninus (1427-1459), Firenze, Olschki, 1995.

J. Hürbin 1898, a c. di, Die Statuten der Juristen-Universität Pavia vom Jabre 1396, Luzern, Buchdruckerei Raber \& Cie., 1898.

G. Ianziti 1988, Humanistic Historiography under the Sforzas. Politics and Propaganda in Fifteenth-Century Milan, Oxford, Oxford University Press, 1988.

S. Iaria 2007, Nuove lauree pavesi del Quattrocento, in Negruzzo 2007, 105-120.

S. Iaria 2010, a c. di, Documenti per la storia dell'Università di Pavia nella seconda metà del '400, III. 1461-1463, Milano, Cisalpino, 2010.

S. Iaria - A. Sottili (2008), a c. di, Lauree pavesi nella seconda metà del '400, III. 14911499, Milano, Cisalpino, 2008.

Informatio Pro Bilio Episcopo 1611-1617 = Informatio Pro illustriss(imo) \& Reverendiss(imo) DD. Io. Baptista Bilio Papiae Episcopo Apostolico, et Imperiali privilegiis Ticinensis Academiae Cancellario, ad demonstrandum de officio praef(ati) Illustriss(imi) D. Episcopi, uti dictae Academiae Cancellarii, ac de amplissima eius auctoritate, et solito a principio errectionis Gymnasii, usque modo babito in omnibus ad eiusdem Gymnasii regimen, et rem facientibus, s.l., s.d. [ma 1611-1617].

F. Inghirami 1843, Storia della Toscana compilata ed in sette epoche distribuita, XII, Fiesole, Poligrafia Fiesolana, 1843.

E. Irace 2003, Itale glorie, Bologna, il Mulino, 2003.

A.C. Jemolo 2005, Lettere a Mario Falco, I. 1910-1927, a c. di M. Vismara Missiroli, Milano, Giuffrè, 2005.

E. Junod 1925, Le XI ème Centenaire de l'Université de Pavie, les 20, 21 et 22 mai 1925, Neuchâtel, Imprimeries réunies Borel et Seiler, 1925. 
J. Krynen - M. Stolleis 2008, a c. di, Science politique et droit public dans les facultés de droit européennes (XIII -XVIII ${ }^{e}$ siècles), Frankfurt am Main, Klostermann, 2008.

M.-M. de la Garanderie 2006, L'éclosion (1515-1530). À l'ombre d'Érasme, in Tuilier 2006a, 125-142.

A. Le Ferron 1550, De rebus gestis Gallorum libri IX, Parisiis, Apud Vascosanum, via Iacobea, ab insigne Fontis, 1550.

Leges Salicae 2008 = F. Baldelli, a c. di, Leges Salicae, Ripuariae, Longobardorum, Baioariorum, Caroli Magni. Commentario all'edizione in facsimile, Modena, il Bulino - Archivio Capitolare di Modena, 2008.

E. Levi 1904-1905, Una contesa di precedenza tra Cremona e Pavia nei secoli XVI, XVII e XVIII, Boll. Soc. Pavese St. Patria 4 (1904), 101-115; 5 (1905a), 3-28; (1905b), 147-190.

D. Magnino 1993, Nota introduttiva, in Sacco 1993, 5-6.

D. Magnino 2000, La Facoltà di Lettere a Pavia tra Ottocento e Novecento, in Storia di Pavia, V, 475-488.

R. Maiocchi 1900, Ticinensia. Noterelle di storia pavese pei sec. XV, XVI, Pavia, Artigianelli, 1900.

P. Majocchi 2001, Pavia medievale negli storici ecclesiastici di fine '800, Boll. Soc. Pavese St. Patria 101 (2001), 49-116.

P. Majocchi 2008, Pavia città regia. Storia e memoria di una capitale medievale, Roma, Viella, 2008.

P. Majocchi 2012, Il quadro politico-istituzionale (secoli V-XIV), in Almum Studium Papiense 1,I, 87-104.

A. Malamani 1980, Università e società nella storiografia dell'età moderna, Annali di Storia Pavese 2-3 (1980), 291-298.

E. Malcovati 1985, Universitatis Ticinensis Saecularia Undecima (20-22 maggio 1925), Boll. Soc. Pavese St. Patria 85 (1985), 243-254.

L. Malusa 2011, Antonio Rosmini per l'unità d'Italia. Tra aspirazione nazionale e fede cristiana, Milano, FrancoAngeli, 2011.

D. Mantovani 2004, Domenico Alfeno Vario professore di Diritto civile (1780-1789): l'immedesimazione polemica nell'antico. Versione accresciuta sulla base di ulteriori documenti, in M.G. di Renzo Villata, a c. di, Formare il giurista. Esperienze nell'area lombarda tra Sette e Ottocento, Milano, Giuffrè, 2004, 173-261.

D. Mantovani 2005, Le vocazioni del "Discorso", in A. Manzoni, Discorso sopra alcuni punti della storia longobardica in Italia, a c. di I. Becherucci, Milano, Centro Nazionale Studi Manzoniani, 2005, XI-LXV.

D. Mantovani 2010, Foscolo professore a Pavia: esortazione alla storia dell'Università, Rivista Storica Italiana 122 (2010), 267-306.

D. Mantovani 2012, L'orazione di Nicolò Scillacio in onore di Ludovico il Moro per l'inaugurazione della sede dell'Università di Pavia (ca. 1488), Rivista Storica Italiana 124 (2012), 291-354.

E. Mantovani - E. Valeriani 2011, a c. di, Arcana Studii Papiensis. I documenti fondativi dell'Università di Pavia da Lotario all'Unità d'Italia. Mostra documentaria, Pavia, Università degli Studi, 2011. 
M. Marcocchi 1975, a c. di, Edizione Nazionale del Carteggio di L.A. Muratori, IV. Carteggio con Francesco Arisi, Firenze, Olschki, 1975.

M. Mari 1999, Il genio freddo. La storiografia letteraria di Girolamo Tiraboschi, Milano, CUEM, 1999.

M. Mariani 1900, Cenni storici intorno all'Università di Pavia, Pavia, Tip. Succ. Bizzoni, 1900.

D. Marrara 1984, La giustificazione del diritto romano comune in alcuni autori dell'età moderna con particolare riguardo al Settecento toscano, Quad. Fior. St. Pensiero Giur. Moderno 13 (1984), 287-330.

M.T. Mazzilli Savini 2011, a c. di, Il cortile di Volta dell'Università di Pavia. Maestri e studenti: arte e memoria, Milano, Skira, 2011.

L. Melve 2007, Inventing the Public Sphere. The Public Debate during the Investiture Contest (c. 1030-1122), Leiden - Boston, Brill, 2007.

G. Melville - K.-S. Rehberg 2004, a c. di, Gründungsmythen, Genealogien, Memorialzeichen. Beiträge zur Institutionellen Konstruktion von Kontinuität, Köln, Böhlau, 2004.

G. Melzi 1848, Dizionario di opere anonime e pseudonime di scrittori italiani o come che sia aventi relazione all'Italia, I (A-G), Milano, Pirola, 1848.

Memorie e Documenti, I-III = Memorie e Documenti per la storia dell'Università di Pavia e degli Uomini più illustri che v'insegnarono, I-III, Pavia, Successori Bizzoni, 1877-1878.

A. Mendo 1655, De iure academico, selectae quaestiones theologicae, morales, iuridicae, bistoricae, E politicae. De academiis, magistratibus, collegiis, professoribus, candidatis, et scholasticis, Salamanticae, Typ. Iosephi Gómez de Los Cubos, 1655.

G. Mengozzi 1924, Ricerche sull'attività della Scuola di Pavia nell'Alto medioevo, Pavia, Tipografia Cooperativa, 1924.

G. Mengozzi 1925, L'origine del diploma di laurea e dell'Università, in Contributi alla storia dell'Università di Pavia, 239-249.

J. Merkel 1850, Die Geschichte des Langobardenrechts. Eine Abhandlung [...] als Beitrag zu Savignys Geschichte des römischen Rechts im Mittelalter, Berlin, W. Hertz, 1850.

S. Meschini 1995, Uno storico umanista alla corte sforzesca. Biografia di Bernardino Corio, Milano, Vita e Pensiero, 1995.

S. Meschini 2001, Bernardino Corio storico del Medioevo e del Rinascimento milanese, in P. Chiesa, a c. di, Le cronache medievali di Milano, Milano, Vita e Pensiero, 2001, 101-173.

U. Meyer-Holz 1989, Collegia Iudicum. Über die Form sozialer Gruppenbildung durch die gelehrten Berufsjuristen im Oberitalien des späten Mittelalters, mit einem Vergleich zu Collegia Doctorum Iuris, Baden Baden, Nomos, 1989.

MGH = Monumenta Germaniae Historica

MGH Capit. 1-2 = Capitularia Regum Francorum, a c. di A. Boretius - V. Krause, Hannoverae, impensis Bibliopolii Hahniani, 1883-1897 (rist. 1984-2001).

MGH Conc. 2, 2 = Concilia, II. Concilia Aevi Karolini, 2, a c. di A. Werminghoff, Hannoverae et Lipsiae, impensis Bibliopolii Hahniani, 1908 (rist. 2003). 
MGH Const. 1 = Constitutiones et acta publica imperatorum et regum inde ab a. DCCCCXI usque ad a. MCXCVII (911-1197), a c. di L. Weiland, Hannoverae, impensis Bibliopolii Hahniani, 1893 (rist. 2003).

MGH Epp. 4 = Epistolarum Tomus IV, Karolini aevi 2, a c. di E. Dümmler et Alii, Berolini, Apud Weidmannos, 1895 (rist. 1994).

MGH LL 4 = Leges, IV. Leges Langobardorum, a c. di F. Bluhme - A. Boretius, Hannoverae, impensis Bibliopolii Aulici Hahniani, 1868 (rist. 1984).

MGH SS 6 = Scriptorum Tomus VI, a c. di G.H. Pertz et Alii, Hannoverae et Lipsiae, impensis Bibliopolii Hahniani, 1844 (rist. 1980).

MGH SS 22 = Scriptorum Tomus XXII [Historici Germaniae saec. XII.], a c. di G.H. Pertz et Alii, Hannoverae, impensis Bibliopolii Hahniani, 1872 (rist. 1976).

MGH SS 30,2 = Scriptorum Tomi XXX Pars II [Supplementa tomorum I-XV], a c. di A. Hofmeister, Lipsiae, impensis K.W. Hiersemann, 1934 (rist. 1976).

MGH SS rer. Germ.n.s. 12 = Scriptores rerum Germanicarum, Nova Series, XII. Notkeri Balbuli, Gesta Karoli Magni Imperatoris, a c. di H.F. Häfele, Berolini, Apud Weidmannos, 1959.

MGH, SS rer. Germ. 25 = Scriptores rerum Germanicarum in usum scholarum separatim editi, XXV. Einhardi, Vita Karoli Magni, a c. di O. Holder-Egger, Hannoverae et Lipsiae, impensis Bibliopolii Hahniani, 1911 (rist. 1965).

J. Middendorp 1572, Academiarum orbis Christiani libri duo. Quibus praeter earum originem, institutionem, E progressiones, Noachi post diluvium, ESS. Apostolorum Iesu Christi in Europam adventus \& Coloniae describuntur, Coloniae, Apud Maternum Cholinum, 1572.

J. Middendorp 1583, Academiarum universi terrarum orbis libri tres. Nunc recens locupletati \& recogniti, Coloniae, Apud Maternum Cholinum, 1583.

J. Middendorp 1602, Academiarum celebrium universi terrarum orbis liber quartus. Qui reliquas a Romana Academias Italiae, E Siciliae edisserit, Coloniae Agrippinae, Apud Gosuinum Cholinum, 1602.

F. Milani 2000, Siro Severino Capsoni, in «...parlano un suon che attenta Europa ascolta». Poeti, scienziati, cittadini, 417-420.

A. Momigliano 1984, Sui fondamenti della storia antica, Torino, Einaudi, 1984.

M. Moretti 2007, Appunti sulla storia della medievistica italiana fra Otto e Novecento: alcune questioni istituzionali, Jerónimo Zurita. Revista de Historia 82 (2007), $155-174$.

L. Moscati 2000, Italienische Reise. Savigny e la scienza giuridica della Restaurazione, Roma, Viella, 2000.

L.A. Muratori 1725, Additamenta ad Leges, sive Capitularia Lotharii I ex Codice Ms. Cathedralis Mutinensis, in Rerum Italicarum Scriptores, I.2, Mediolani, Ex typographia Societatis Palatinae, 1725.

L.A. Muratori 1751, Dissertazioni sopra le antichità italiane, I-III, Milano, a spese di G. Pasquali, 1751.

A.M. Nada Patrone 1962, Azario, Pietro, DBI 4 (1962), 740-742.

P. Nardi 1992, Le origini del concetto di Studium Generale, Rivista Internazionale di Diritto Comune 3 (1992), 47-78. 
P. Nardi 2007, Gli Studi Generali e la formazione degli statuti universitari medievali, in Romano 2007, 49-59.

P. Nardi 2008, L'Archivio di Stato di Siena e il mondo universitario tra Ottocento e Novecento, Annali di Storia delle Università Italiane 12 (2008), 469-480.

E. Necchi 1994, Le iscrizioni di Raffaele Fulgosio e Raffaele Raimondi, maestri giuristi, fra Pavia e Padova, Italia Medioevale e Umanistica 37 (1994), 215-222.

S. Negruzzo 2001, L'Archivio storico dell'Università di Pavia depositato presso l'Archivio di Stato di Pavia, Annali di Storia Pavese 29 (2001), 75-81.

S. Negruzzo 2007, a c. di, Università, umanesimo, Europa. Giornata di studio in ricordo di Agostino Sottili (Pavia, 18 novembre 2005), Milano, Cisalpino, 2007.

A. Nova 1862, La filosofia. La filosofia del diritto e l'Università. Prolusioni lette nell'Università di Pavia negli anni 1854 e 1859, Milano, Tip. Pietro Agnelli, 1862.

Ottocento e Novecento 1984 = Ottocento e Novecento nelle collezioni d'arte dei Civici musei di Pavia, Pavia, Comune di Pavia, 1984.

A. Padoa Schioppa, La Scuola di Pavia. Alle fonti della nuova scienza giuridica europea, in Almum Studium Papiense 1,I, 143-164.

A. Padovani 2007, Roberto di Torigni, Lanfranco, Irnerio e la scienza giuridica anglo-normanna nell'età di Vacario, Rivista Internazionale di Diritto Comune 18 (2007), 71-140.

B. Pagnin 1969, Scuola e cultura a Pavia nell'alto Medioevo, in Pavia capitale di Regno, 75-106.

G. Panciroli 1637, De claris legum interpretibus. Libri quatuor, Venetiis, Apud Marcum Antonium Brogiollum, 1637.

«...parlano un suon che attenta Europa ascolta». Poeti, scienziati, cittadini $=$ «...parlano un suon che attenta Europa ascolta». Poeti, scienziati, cittadini nell'ateneo pavese tra Riforme e Rivoluzione, Pavia, Tip. commerciale pavese, 2000.

G. Parodi 1753, Elenchus privilegiorum, et actuum publici Ticinensis Studii a saeculo nono, ad nostra tempora collectus, et seriatim expositus, diligentia, studio, et sumptibus J.C. Jacobi Parodii in eodem patrio Archygimnasio publici Pandectarum professoris. Cui accedit Sylabus [sic] Lectorum, praestantiorumque virorum eiusdem Studii stipendiis emaeritorum; Elenchus egregiorum a secretis, privilegiis, et actibus ipsis subsignatorum, necnon, et Series Lectorum, qui in prima sede utriusque Juris professi sunt; et novissime Historico-Juridica probatio de praestantia eiusdem cathedrae Pandectarum, [Pavia], s.e., 1753.

A. Pasi Testa 1980, Bernardo Sacco e il paesaggio agrario e urbano pavese nel Cinquecento, Annali di Storia Pavese 2-3 (1980), 229-250.

M. Paulmier-Foucart 2004, Vincent de Beauvais et le Grand miroir du monde, Turnhout, Brepols, 2004.

Pavia capitale di Regno = Pavia capitale di Regno. Atti del IV Congresso internazionale di Studi sull'Alto Medioevo (Pavia - Scaldasole - Monza - Bobbio, 10-14 settembre 1967), Spoleto, CISAM, 1969.

C. Pecorella 1964, Studi sul Settecento giuridico, I. L.A. Muratori e i difetti della giurisprudenza, Milano, Giuffrè, 1964.

S. Perondoli 1808, De Napoleone M. apud Insubres scientiarum et bonarum artium vindice atque statore. Adumbratio, Ticini, Ex Typographia Bolzani, 1808. 
B. Peroni 1925, La riforma dell'Università di Pavia nel settecento, in Contributi alla storia dell'Università di Pavia, 115-174.

A. Petrucci 1967, Beretti, Giovanni Gaspare, DBI 9 (1967), 59-61.

G. Petti Balbi 1988, Le università medioevali, in La Storia. I grandi problemi dal medioevo all'eta contemporanea, I.1. Il Medioevo, Torino, UTET, 1988, 579-600.

É. Picot 1915, Les professeurs et les étudiants de langue française à l'Université de Pavie au XVe et au XVI ${ }^{e}$ siècle, Bulletin Philologique et Historique (jusqu'à 1715) du Comité des Travaux Historiques et Scientifiques (1915), 8-90 (ed. Paris, Imprimerie Nationale, 1916).

Pietro da Castelletto 1730, Sermo [...] in exequiis [...] Johannis Galeaz, a c. di L.A. Muratori, in Rerum Italicarum Scriptores, XVI, Mediolani, Ex typographia Societatis Palatinae, 1730, coll. 1038-1050.

H. Pignot 1970, Jurisconsulte au XVI siècle. Barthélemy de Chasseneuz, premier commentateur de la coutume de Bourgogne et Président du Parlement de Provence, sa vie et ses œuvres, Genève, Slatkine, 1970 (ed. orig. 1880).

F. Piovan 2007, Maestri pavesi nello Studio di Padova nel terzo e quarto decennio del Cinquecento, in Negruzzo 2007, 79-95.

P. Pissavino 1986, Modelli di scrittura politica a Pavia nell'età della Controriforma. Note su Enrico Farnese, Boll. Soc. Pavese St. Patria 86 (1986), 83-106.

P. Pissavino 2013, Università e Accademie, in Almum Studium Papiense, 1,II, 1223-1258.

M. Poletti 2009, «Pare una Roma». Un segretario di Baldassar Castiglione nella Pavia del 1523, Boll. Soc. Pavese St. Patria 109 (2009), 327-333.

G. Politi 2002, La società cremonese nella prima età spagnola, Milano, Unicopli, 2002.

A. Potthast 1859, a c. di, Liber de rebus memorabilioribus sive chronicon Henrici de Hervordia, Gottingae, sumptibus Dieterichianis, 1859.

T.C. Price Zimmermann 1995, Paolo Giovio. The Historian and the Crisis of SixteenthCentury Italy, Princeton, Princeton University Press, 1995.

Ch.M. Radding 1988, The Origins of Medieval Jurisprudence: Pavia and Bologna (8501150), New Haven - London, Yale University Press, 1988.

Ch.M. Radding - A. Ciaralli 2007, The Corpus Iuris Civilis in the Middle Ages. Manuscripts and Trasmission from the Sixth Century to the Juristic Revival, Leiden - Boston, Brill, 2007.

P. Radiciotti 1996, Felice, DBI 46 (1996), 25-26.

E. Raimondi 1989, I Lumi dell'erudizione. Studi sul Settecento italiano, Milano, Vita e Pensiero, 1989.

M. Randall 2008, The Gargantuan Polity. On the Individual and the Community in the French Renaissance, Toronto - Buffalo - London, University of Toronto Press, 2008.

H. Rashdall 1936, The Universities of Europe in the Middle Ages, a c. di F.M. Powicke - A.B. Emden, I-III, Oxford, Clarendon Press, 1936 (I ed. 1895; repr. 1951).

M.C. Regali 1999, Le Ricerche storiche sull'Accademia degli Affidati di Siro Comi. Edizione delle postille d'autore, Boll. Soc. Pavese St. Patria 99 (1999), 167-260.

M.C. Regali 2000, Siro Comi, in «... parlano un suon che attenta Europa ascolta». Poeti, scienziati, cittadini, 400-405.

C. Repossi 1995, La cultura letteraria a Pavia nei secoli XVI-XVIII, in Storia di Pavia, IV.2, 689-746. 
C. Repossi 2013, L'Accademia degli Affidati e l'Università dal secolo XVI al XVIII, in Almum Studium Papiense, 1,II, 1259-1262.

Responsa, et Instructiones 1612 = Responsa, et Instructiones in causis Immunitatis Lectorum Gymnasii Ticinensis, Milano, nella Regia Ducal Corte, Per Pandolfo e Marco Tullio Malatesti, 1612.

G. Robolini 1823-1838, Notizie appartenenti alla storia della sua patria, I-VI, Pavia, Fusi, 1823-1838.

A. Romano 2007, a c. di, Gli statuti universitari. Tradizione dei testi e valenze politiche. Atti del Convegno internazionale di Studi (Messina - Milazzo, 13-18 aprile 2004), Bologna, CLUEB, 2007.

P. Rosso 2012, Professori, studenti e nationes, in Almum Studium Papiense 1,I, 383-414.

P.L. Rovito 1990, De Luca, Carlo Antonio, DBI 38 (1990), 333-334.

R. Ruggeri 2000, Polidoro Virgili. Un umanista europeo, Bergamo, Moretti \& Vitali, 2000.

L. Rustenius 1550, Catalogus Academiarum orbis Christiani, Augustae Vindelicorum, Philippus Ulhardus excudebat, 1550.

M.A. Sabellico 1528, Rapsodia Historiarum Enneadum ab orbe condito in annum usque salutis nostrae 1504. Posterior pars [...], continens sex Enneades reliquas cum earundem repertoriis E epitomis, Parisiis, In aedibus Ascensianis, 1528.

B. Sacco 1587, De Italicarum rerum varietate, et elegantia, libri X. In quibus multa scitu digna recensentur de populorum vetustate, dominio, et mutatione [...]. Eiusdem De Papiensis Ecclesiae dignitate, nulli metropolitano suppositae, enarratio [...]. Ad haec, De dignitate Gymnasii Ticinensis Henrici Farnesii Eburonis encomium, Ticini, Apud Hieronymum Bartolum, 1587.

B. Sacco 1993, Storia di Pavia, trad. di Magnino Domenico, Como, New Press, 1993.

Saecularia Undecima = Universitatis Ticinensis Saecularia Undecima. Die 21 maii mensis anno 1925, Pavia, Bruni-Marelli, 1925.

P. Sangiorgio 1831, Cenni storici sulle due università di Pavia e di Milano e notizie intorno ai più celebri medici, chirurghi e speziali di Milano dal ritorno delle scienze fino all'anno 1816. Opera postuma, a c. di F. Longhena, Milano, Placido Maria Visaj, 1831.

F.C. von Savigny 1834-1851, Geschichte des römischen Rechts im Mittelalter, I-VI, Heidelberg, Mohr, 1834-1851.

J. Schneider 1997, Vincent de Beauvais à l'épreuve des siècles, in S. Lusignan - M. Paulmier-Foucart, a c. di, Lector et compilator. Vincent de Beawvais, frère prêcheur un intellectuel et son milieu au XII siècle, Grâne, Créaphis, 1997, 21-46.

A.A. Settia 1997, Il sogno regio dei Visconti, Pavia e la Certosa, in La Certosa di Pavia tra devozione e prestigio dinastico. Atti del Convegno di studi (Pavia - Certosa, 1618 maggio 1996), (Annali di Storia Pavese, 1997), 13-15.

A.A. Settia 2001, Giacinto Romano, "uomo di studio e di battaglia", Boll. Soc. Pavese St. Patria 101 (2001), 9-15.

E. Signori 2002, Minerva a Pavia. L'ateneo e la città tra guerre e fascismo, Milano, Cisalpino, 2002.

F. Šmahel 2007, Magister und Studenten der Prager medizinischen Fakultät bis zum Jahre 1419, in Id., Die Prager Universität im Mittelalter. Gesammelte Aufsätze, Leiden, Brill, 2007, 103-158. 
G.F. Soli Muratori 1773, Vita del proposto Ludovico Antonio Muratori, Napoli, Giovanni Gravier, 1773.

A. Solmi 1920a, Il testo delle Honorantie civitatis Papie, Archivio Storico Lombardo 47 (1920), 177-192.

A. Solmi 1920b, Giacinto Romano, Boll. Soc. Pavese St. Patria 20 (1920), 127-137.

A. Solmi 1925, La persistenza della scuola di Pavia nel medio evo fino alla fondazione dello Studio Generale (1024-1361), in Contributi alla storia dell'Università di Pavia, 15-42.

A. Solmi 1932, L'amministazione finanziaria del Regno Italico nell'alto Medio Evo. Col testo delle "Honorantie civitatis Papie" e con una appendice di XVIII documenti, Pavia, Tip. Cooperativa, 1932.

R. Sòriga 1924, Rodolfo Maiocchi, Boll. Soc. Pavese St. Patria 24 (1924), 231-233.

A. Sottili 1983, Per una continuazione del Codice diplomatico dell'Università di Pavia nella seconda metà del Quattrocento: documenti milanesi, Wolfenbütteler Renaissancemitteilungen 7 (1983), 146-151.

A. Sottili 1994, a c. di, Documenti per la storia dell'Università di Pavia nella seconda metà del' '400, I. 1450-1455, Milano, Cisalpino, 1994.

A. Sottili 1995, a c. di, Lauree Pavesi nella seconda metà del '400, I. 1450-1475, Milano, Cisalpino, 1995.

A. Sottili 1998, a c. di, Lauree Pavesi nella seconda metà del '400, II. 1476-1490, Milano, Cisalpino, 1998.

A. Sottili - P. Rosso 2002, a c. di, Documenti per la storia dell'Università di Pavia nella seconda metà del '400, II. 1455-1460, Milano, Cisalpino, 2002.

G. Spiegel 1997, The Past as Text. The Theory and Practice of Medieval Historiography, Baltimore - London, The Johns Hopkins University Press, 1997.

Statuti e Ordinamenti 1925 = Statuti e Ordinamenti della Università di Pavia dall'anno 1361 all'anno 1859, raccolti e pubblicati nell'XI centenario dell'Ateneo, Pavia, Tipografia Cooperativa, 1925.

P. Stella 2008, Il libro Religioso in Italia. Studi e ricerche, Roma, Viella, 2008.

H.S. Stone 2002, St. Augustine's bones. A microhistory, Amherst - Boston, University of Massachussets Press, 2002.

Storia di Pavia = Storia di Pavia, a c. di E. Gabba, I-V, Milano, Banca del Monte di Lombardia, 1984-2000.

C. Storti Storchi 2007, Scritti sugli statuti lombardi, Milano, Giuffrè, 2007.

P. Terenzio 1861, Intorno alla riduzione e riforma delle Università in Italia e cenno storico sull'Università di Pavia, Pavia, Fusi, 1861.

G. Tiraboschi 1773, III, Storia della letteratura italiana, III. Dalla Rovina dell'Impero Occidentale fino all'Anno MCLXXXIII, Modena, Società Tipografica, 1773.

G. Tiraboschi 1775, V, Storia della letteratura italiana, V. Dall'anno MCCC fino all'anno MCCCC, Modena, Società Tipografica, 1775.

G. Tiraboschi 1777, VII.1, Storia della letteratura italiana, VII. Dall'anno MD fino all'anno MDC. Parte I, Modena, Società Tipografica, 1777.

G. Tiraboschi 1787, III, Storia della letteratura italiana, III. Dalla rovina dell'Impero occidentale fino all' anno MCLXXXIII, Modena, Società Tipografica $1787^{2}$.

P. Tomea 1993, Tradizione apostolica e coscienza cittadina a Milano nel medioevo. La leg- 
genda di san Barnaba, Milano, Vita e Pensiero, 1993.

D. Tongiorgi 1997, L'eloquenza in cattedra. La cultura letteraria nell'Università di Pavia dalle riforme teresiane alla Repubblica Italiana (1769-1805), Milano, Cisalpino, 1997.

E. Tremp 2009, Menschliche Größe und Schwäche bei Notker Balbulus († 912), in A. Härter, a c. di, Liebe und Zorn. Zu Literatur und Bucbkultur in St. Gallen, Wiesbaden, Harrassowitz, 2009, 15-40.

A. Tuilier 1981, La notion Romano-byzantine de studium generale et les origines des nations dans les universités médiévales, Bulletin Philologique et Historique du Comité des travaux Historiques et Scientifiques (1981), 7-27.

A. Tuilier 2006a, a c. di, Histoire du College de France, préface de M. Fumaroli, Paris, Fayard, 2006.

A. Tuilier 2006b, Introduction bistorique générale, in Id. 2006a, 7-52.

A. Tuilier 2006c, L'entrée en fonction des premiers lecteurs royaux, in Id. 2006a, 145-163.

S. Twardzik 2007, Le vicende istituzionali dell'Università degli studi di Milano dalla sua fondazione agli anni Sessanta del Novecento, Annali di Storia delle Università Italiane 11 (2007), 45-63.

G.M. Varanini 1986, Dal comune allo stato regionale, in La Storia. I grandi problemi dal Medioevo all'Età Contemporanea, II. Il Medioevo, 2. Popoli e strutture politiche, Torino, UTET, 1986, 693-724.

G.M. Varanini 2007, I riti dell'assedio. Alcune schede dalle cronache tardomedievali, Reti Medievali. Rivista 8 (2007), on line.

C. Varotti 1998, Gloria e ambizione politica nel Rinascimento. Da Petrarca a Machiavelli, Milano, Bruno Mondadori, 1998.

P. Vergilio 1570, Anglicae historiae libri vigintiseptem, Basileae, Apud Thomam Guarinum, 1570.

M.G. Vida 1550, Cremonensium orationes III adversus Papienses in controversia principatus, Cremonae, [Johannes Mutius et Bernardinus Locheta], 1550.

M.G. Vida 1975, The game of chess. Marco Girolamo Vidas "Scacchia Ludus", a c. di M.A. Di Cesare, Nieuwkoop, B. De Graaf, 1975.

M.G. Vida 1982, L'arte poetica, a c. di R. Girardi, Bari, Adriatica, 1982.

A.T. Villa 1779, De bistoria Gymnasii Ticinensis perscribenda. Oratio babita Ticini in Templo Maximo III Id. Novembris a. 1779 Archigymnasii Instaurati decimo, Ticini Regii, Typis R.\&.I. Monasterii S. Salvatoris per Josepehum Blancum, 1779.

A.T. Villa 1782, De studiis literariis Ticinensium ante Galeatium II Vicecomitem sive ad historiam Gymnasii Ticinensis prodromus, Ticini, in Typographeo Monasterii S. Salvatoris, 1782.

C. Viola 1999, Edizione Nazionale del Carteggio di L.A. Muratori, XXVIII. Carteggi con Mansi [...] Marmi, Firenze, Olschki, 1999.

A. Visconti 1925, L'opera del Governo Austriaco nella riforma universitaria durante il ventennio 1753-1773, in Contributi alla storia dell'Università di Pavia, 175-237.

A. Vivanti 1956, L'ultima accademia pavese: [discorso tenuto] al Rotary Club di Pavia il 19 dicembre 1956, Pavia, s.n.t.

M. Volpi 2000, Giuseppe de' Necchi Aquila, in «... parlano un suon che attenta Europa ascolta». Poeti, scienziati, cittadini, 407-412. 
Z. Volta 1890, Dei gradi accademici conferiti nello "Studio generale" di Pavia sotto il dominio visconteo, Archivio Storico Lombardo 18 (1890), 517-584.

H.G. Walther 2008, Pavia und Padua im frühen 15. Jabrhundert. Zur Profilierung zweier Rechtsuniversitäten bei der Ausbildung für die politische Praxis, in Krynen Stolleis 2008, 263-282.

F. Zabarella 1602, In Clementinarum volumen commentaria, Venetiis, Apud Iuntas, 1602.

N. Zacour 1957, Stephanus Hugoneti and his "Apparatus" on the Clementines, in Traditio 13 (1957), 456-462.

H. Zedelmaier 2003, Der Anfang der Geschichte. Studien zur Ursprungsdebatte im 18. Jabrbundert, Hamburg, Felix Meiner, 2003.

M.C. Zorzoli 1986, Università, dottori, giureconsulti. L'organizzazione della facoltà legale di Pavia nell'età spagnola, Padova, CEDAM, 1986.

M.C. Zorzoli 1995, La Facoltà di giurisprudenza (1535-1796), in Storia di Pavia, IV.1, 483-516.

M.C. Zorzoli 2000, Docenti dell'Università di Pavia tra Sei e Settecento: gli uomini, le idee tra diritto locale ed erudizione, Annali di Storia Moderna e Contemporanea 6 (2000), 359-390. 